\title{
A Study of Kripke-type Models for Some Modal Logics by Gentzen's Sequential Method
}

\author{
By
}

\author{
Masahiko SATo*
}

\section{TABLE OF CONTENTS}

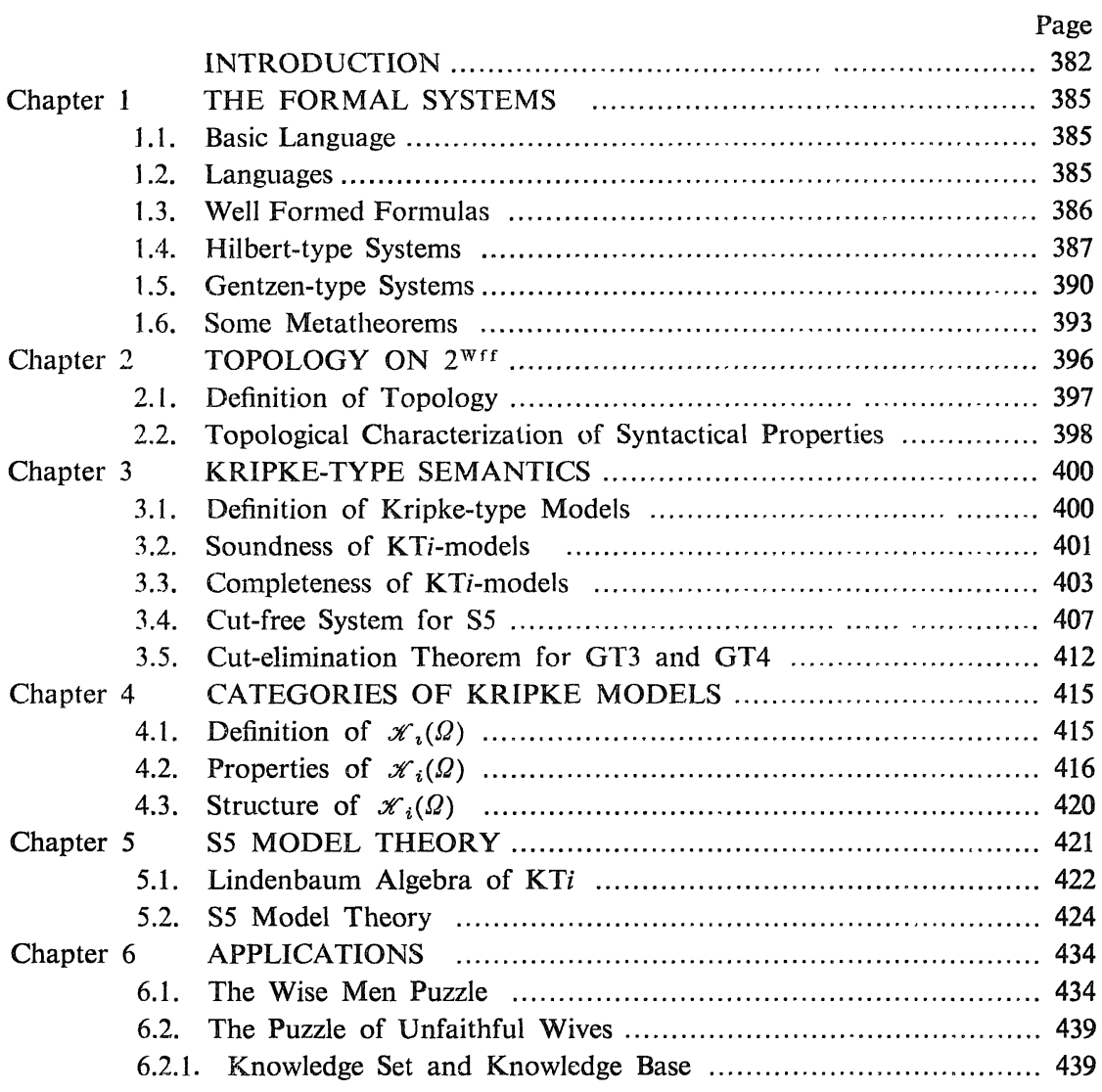

Received July 26, 1976.

* Research Institute for Mathematical Scicnces, Kyoto University, Kyoto 606, Japan. Present address: Department of Mathematics, College of General Education, University of Tokyo, Komaba, Tokyo 153, Japan. 


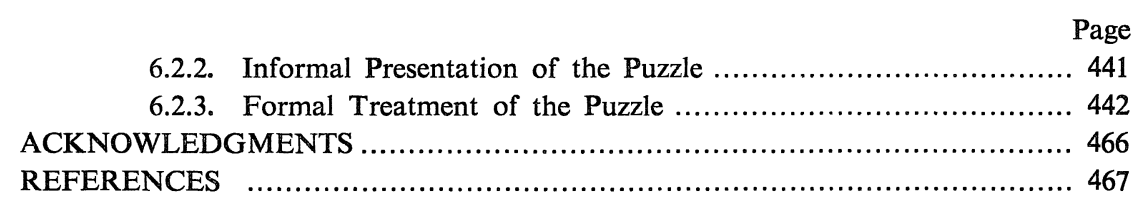

\section{Introduction}

The main objective of the present paper is to clarify a close relationship between Gentzen-type sequential formulation of formal systems (especially of modal calculi) and Kripke-type semantics. Though the investigations by Schütte [31], Maehara [20], Fitting [3], Prawitz [27], etc. have suggested this relationship either explicitly or implicitly, the usefulness of Gentzen systems for the semantical studies of modal calculi seems to be less recognized than it deserves. In this paper, we wish to establish its usefulness in a decisive way. We now proceed to explain the background motivation for our study.

When an interpretation, or semantics, of a formal system is given, we are always interested in the question: "Is it complete?" Indeed, the completeness of the semantics is essential so that it is really useful for the study of the formal system in question. The naturalness of the semantics is fundamental as well. For instance, in the case of modal calculi, we know such semantics as algebraic, topological and Kripketype. (See Cresswell [2], Lemmon [18], Rasiowa [28], Rasiowa-Sikorski [29], Segerberg [34] etc.) Among these, Kripke-type semantics introduced by Kripke $[15,16]$ has proved to be most successful.

On the other hand, the method of formulating a formal system is not unique. Formulations such as Hilbert-type, natural deduction, Gentzen's sequent system and Smullyan's analytic tableau are well-known. And each formulation has its own merits for both syntactical and semantical study of formal systems. (See, e.g., Kreisel [13, 14], Prawitz [25, 26], Zucker [39], Takeuti [38] and Smullyan [35].) In this paper, however, we take the standpoint of regarding that Gentzen-type sequential formulation is best fitted for the Kripke-type semantical study of formal systems. We have slightly modified the notion of a sequent in order to establish the natural correspondence between Gentzen systems and Kripke models. I.e., we define a sequent as a pair of two (possibly 
infinite) sets of well formed formulas.

Though our method is general enough to admit applications to, for example, intermediate logics and other modal calculi, we will, in this paper, only concentrate on three modal systems KT3, KT4 and KT5 of knowledge as introduced by McCarthy [21, 22]. However, since these systems are generalizations of bi-modal logics S4-T, S4-S4 and S5-S5, which in turn are generalizations of $\mathrm{T}, \mathrm{S} 4$ and $\mathrm{S} 5$, our results apply directly to these modal calculi. In fact, we have so designed the languages that our argument will always be relative to a particular choice of the language, and that by a suitable choice of the language we will be able to obtain the specific result for any one of these logics. We leave applications of our method to other logics to the interested reader.

There are many known proof-techniques of completeness results. See, e.g., Gödel [6], Henkin [10], Takahashi [37], Fitting [3], Smullyan [35], Kripke [15, 16], Lemmon-Scott [18], Segerberg [34], Schütte [31] and Maehara [20]. In the present paper, we prove the completeness theorem in two different ways. The first one is the so-called Henkinstyle proof. However, our proof is new in that it is relative to a set $\Omega$ of wffs which is closed under subformulas, so that we can at the same time prove compactness by letting $\Omega$ to be the whole set of wffs and decidability by letting $\Omega$ to be the set of subformulas of a certain formula. Our second proof is based on cut-free formulations of the systems. Especially, a cut-free system for S5 is obtained by a close inspection of the first proof. The cut-elimination theorem of these systems yields our second proof of the decidability of KT3, KT4 and S5. For KT3 and $\mathrm{KT} 4$, it also gives a proof of the disjunction property of these logics.

As we mentioned above, in our first proof of the completeness theorem, we construct a model $U(\Omega)$, called the universal model over $\Omega$, for any $\Omega$ which is closed under subformulas. By means of this fundamental model, we will define a category $\mathscr{K}(\Omega)$ of Kripke-type models over $\Omega$. In this category, $U(\Omega)$ will be characterized as "the" terminal object of the category. The classification problem of models will also be conveniently treated in this category. For the modal logic S5, we can obtain a complete classification of models. This result easily shows the normal form theorem for S5, and the structure of Lindenbaum 
algebra of S5 will also be determined.

We now briefly sketch the content of each chapter.

In Chapter 1, we first define the languages upon which our formal systems will be built. The main reason for introducing many languages rather than a single language is that we can explain the difference between certain logics (such as S4 and S4-T) as the mere difference of languages. We then define Hilbert-type axiomatizations of the three modal systems KT3, KT4 and KT5. Corresponding to these, three equivalent Gentzen-type sequential systems GT3, GT4 and GT5 will be defined. Though our notion of a sequent admits an infinite set of wffs both in the antecedent and in the succedent, a theorem to the effect that this generalization is superficial will be proved. Nevertheless, the importance of the generalization will be fully exhibited in the subsequent chapters.

In Chapter 2, we introduce a topology, which is homeomorphic to Scott's $P \omega$ topology, on $2^{\text {wff }}$, where Wff is the set of wffs. Several syntactic notions concerning deducibility will be expressed in topological terminology.

In Chapter 3, we define the Kripke-type semantics for $\mathrm{KT} i(i=3$, 4, 5). Two completeness proofs will be given there. Compactness, decidability and cut-elimination theorem will be proved as by-products. The first completeness proof furnishes us with a basis for subsequent studies, while the importance of the second proof lies in giving cut-free systems as by-products.

Chapter 4 is devoted to the category theory of Kripke models. In contrast to the notion of $p$-morphism due to Segerberg [34], which is defined by referring to the relational structure of models, our notion of homomorphism is defined without any explicit reference to the relational structure of models. Roughly speaking, we define an $(\Omega$-) homomorphism as a mapping which preserves the semantics in $U(\Omega)$ of a model. Thus for each $\Omega$, we obtain a distinct category $\mathscr{K}(\Omega)$. In case $\Omega$ is equal to Wff, our notion of homomorphism contains the notion of $p$-morphism.

In Chapter 5, we study the modal calculus S5 as an application of the results obtained in Chapter 4. A complete classification of S5 models under a certain equivalence relation on models will be given. Our method gives another proof of normal form theorems by Itoh [12] and 
the result of Bass [1] which determines the Lindenbaum algebra of S5 with finite generators.

The final chapter, Chapter 6 , is devoted to the study of two wellknown puzzles, the puzzle of wise men and the puzzle of unfaithful wives. It was McCarthy [21] who first attacked these puzzles in a formal manner. The second puzzle, however, remained almost untouched. The difficulties which arise in the formal presentation of the puzzle are twofold. Firstly, the puzzle involves the self-referential statements. Secondly, the totality of one's knowledge is difficult to characterize. We will present a solution which we think successfully gets over these difficulties. The notion of knowledge set and knowledge base to be defined in this chapter will play an important role in characterizing the totality of one's knowledge. A model-theoretic solution of the puzzle of wise men will also be given there.

\section{Chapter 且}

\section{The Formal Systems}

\subsection{Basic Language}

The basic language $\mathbb{L}$ is a triple $\left(\mathbb{P r}, \boldsymbol{S p}, \mathbb{N}^{+}\right)$, where

$$
\begin{aligned}
& \mathbb{P r}=P_{1}, P_{2}, \ldots ; \\
& S_{P}=S_{0}, S_{1}, \ldots ; \\
& \mathbb{N}^{+}=\overline{1}, \overline{2}, \ldots
\end{aligned}
$$

are denumerable sequences of distinct symbols. $\mathbb{N}^{+}$is the set of numerals denoting the corresponding positive integers. But, for simplicity, we will identify $\overline{\mathrm{n}}$ with the integer $n . S_{0} \in S_{P}$ will also be denoted by $O$ and will be called "FOOL."

\subsection{Languages}

A language $L$ is a triple $(\operatorname{Pr}, S p, T)$ where

$$
\operatorname{Pr} \subseteq \boldsymbol{P r}
$$




$$
\begin{gathered}
S p \subseteq \mathbf{S p} \\
T \subseteq \mathbf{N}^{+} .
\end{gathered}
$$

Elements in $\operatorname{Pr}, S p$ and $T$ denote propositional variables, persons and time, respectively. Our arguments henceforth will, unless stated otherwise, always be relative to a language $L$. So the reader may choose any language he likes and read the following by fixing his favorite language. For example, if he is only interested in the classical propositional calculus, he should take $L=(\boldsymbol{P r}, \emptyset, \emptyset)$. When an explicit mention of the language $L$ to be considered is necessary, we will express it by explicitly writing $L$ somewhere as a suffix etc.

\subsection{Well Formed Formulas}

The set of well formed formulas is defined to be the least set Wff such that:

(W1) $\perp \in$ Wff;

(W2) $\quad \operatorname{Pr} \subseteq \mathrm{Wff}$;

(W3) $\alpha, \beta \in$ Wff implies $\supset \alpha \beta \in$ Wff;

(W4) $S \in S p, t \in T, \alpha \in$ Wff implies $S t \alpha \in$ Wff.

The symbols $\perp$ and $\supset$ denote "false" and "implication", respectively.

We will make use of the following abbreviations:

$$
\begin{array}{ll}
\alpha \supset \beta=\supset \alpha \beta & \text { read " } \alpha \text { implies } \beta \text { " } \\
\neg \alpha=\alpha \supset \perp & \text { read "not } \alpha \text { " } \\
T=\neg \perp & \text { read "true" } \\
\alpha \vee \beta=\neg \alpha \supset \beta & \text { read " } \alpha \text { or } \beta \text { " } \\
\alpha \wedge \beta=\neg(\alpha \supset \neg \beta) & \text { read " } \alpha \text { and } \beta \text { " } \\
{[S t] \alpha=S t \alpha} & \text { read "S knows } \alpha \text { at time } t \text { " } \\
<S t>\alpha=\neg[S t] \neg \alpha & \text { read " } \alpha \text { is possible for } S \text { at time } t \text { " } \\
\{S t\} \alpha=[S t] \alpha \vee[S t] \neg \alpha & \text { read "S } S \text { knows whether } \alpha \text { at time } t \text { " }
\end{array}
$$

Remark. If $L$ is the simplest language $(\varnothing, \varnothing, \emptyset)$, the conditions (W2) and (W4) in the definition of Wff become vacuous, so that we have $\mathrm{Wff}=\{\perp, \perp \supset \perp, \perp \supset(\perp \supset \perp),(\perp \supset \perp) \supset \perp, \ldots\}$. We will not repeat this 
sort of remarks in the sequel. However, the reader should always be alert and notice that the definitions or proofs may become simpler for a particular choice of $L$. We also remark that the cardinality of Wff is $\omega$ irrespective of $L$.

For any $\alpha \in \mathrm{Wff}$, we define $\operatorname{Sub}(\alpha) \subseteq \mathrm{Wff}$ inductively as follows:

(S1) $\alpha \in \operatorname{Pr} \cup\{\perp\} \Rightarrow \operatorname{Sub}(\alpha)=\{\alpha\}$;

(S2) $\alpha=\beta \supset \gamma \Rightarrow \operatorname{Sub}(\alpha)=\{\alpha\} \cup \operatorname{Sub}(\beta) \cup \operatorname{Sub}(\gamma)$;

(S3) $\alpha=[S t] \beta \Rightarrow \operatorname{Sub}(\alpha)=\{\alpha\} \cup \operatorname{Sub}(\beta)$.

We say $\beta$ is a subformula of $\alpha$ if $\beta \in \operatorname{Sub}(\alpha)$.

\subsection{Hilbert-type Systems}

We now define three modal systems KT3, KT4 and KT5 of knowledge due to McCarthy [22]. We begin with the definition of KT3.

The axiom schemata for KT3 are:

(A1) $\neg \neg \alpha \supset \alpha$

(A2) $\alpha \supset(\beta \supset \alpha)$

(A3) $(\alpha \supset(\beta \supset \gamma)) \supset((\alpha \supset \beta) \supset(\alpha \supset \gamma))$

(A4) $[S t] \alpha \supset \alpha$

(A5) $[O t] \alpha \supset[O t][S t] \alpha$

(A6) $[S t](\alpha \supset \beta) \supset([S u] \alpha \supset[S u] \beta)$, where $t \leq u^{1)}$

In (A1)-(A6), $\alpha, \beta, \gamma$ denote arbitrary wffs, $S$ denotes arbitrary element in $S p$, and $t, u$ denote arbitrary elements in $T$.

The notion of a proof in KT3 is defined by:

Definition 1.1. Let $\alpha \in$ Wff. A finite sequence of wffs $\alpha_{1}, \ldots, \alpha_{n}$ ( $n \geq 1)$ is a proof of $\alpha$ in KT3 if $\alpha_{n}=\alpha$ and for each $i$ one of the following three conditions holds:

(i) $\alpha_{i}$ is an instance of (A1)-(A6)

(ii) there exist $j, k<i$ such that $\alpha_{k}=\alpha_{j} \supset \alpha_{i}$ (In this case, we say $\alpha_{i}$ is obtained from $\alpha_{j}$ and $\alpha_{j} \supset \alpha_{i}$ by modus ponens.)

1) $\leq$ denotes the usual ordering of natural numbers. 
(iii) there exists $j<i$ such that $\alpha_{i}=[S t] \alpha_{j}$ for some $S \in S p$ and $t \in T$ (In this case, we say $[S t] \alpha_{j}$ is obtained from $\alpha_{j}$ by ([St]-) necessitation.)

We write $\vdash \alpha$ if there exists a proof of $\alpha$. When we wish to emphasize that it is a proof in KT3, we write $\vdash \alpha$ (in KT3). Furthermore, for any $\Gamma \subseteq$ Wff we write $\Gamma \vdash \alpha$ if $\vdash \beta_{1} \supset\left(\beta_{2} \supset\left(\cdots \supset\left(\beta_{m} \supset \alpha\right) \cdots\right)\right)$ for some $\beta_{1}, \ldots$, $\beta_{m} \in \Gamma$.

It is easy to show the following

Lemma 1.2. Let KT3* be the logical system obtained from KT3 by replacing (A6) by the following two axiom schemata:

(*) $[S t] \alpha \supset[S u] \alpha$, where $t \leq u$

$(* *) \quad[S t] \alpha \wedge[S t](\alpha \supset \beta) \supset[S t] \beta$

Then KT3 and $\mathrm{KT}^{*}$ are equivalent. I.e., for any $\alpha \in \mathrm{Wff}$,

$$
\vdash \alpha(\text { in } \mathrm{KT} 3) \text { iff } \vdash \alpha\left(\text { in } \mathrm{KT} 3^{*}\right) \text {, }
$$

where the notion of a proof in $\mathrm{KT}^{*}$ is defined similarly as in Definition 1.1.

Now, KT4 is defined to be the system obtained from KT3 by adding the following

(A7) $[S t] \alpha \supset[S t][S t] \alpha$

This axiom will be referred to as the positive introspective axiom.

KT5 is obtained by adjoining the following

(A8) $\neg[S t] \alpha \supset[S t] \neg[S t] \alpha$

This axiom will be called the negative introspective axiom.

Remarks.

(1) Axioms (A1)-(A3) give an axiomatization of classical propositional calculus. (See, e.g., Lyndon [19].) Axioms (A4)-(A6) may be intuitively understood as follows.

(A4): What is known is true. 
(A5): What FOOL knows at time $t$, FOOL knows at time $t$ that everyone knows it at time $t$.

(A6): The meaning of (A6) is better explained in terms of (*) and (**) in Lemma 1.2.

(*): What is known remains to be known.

(**): Everybody can do modus ponens.

(2) If $S p$ contains $O$, the condition (iii) of Definition 1.1 may be restricted to: Infer $[O t] \alpha$ from $\alpha$.

(3) The relation of the systems $\mathrm{KT} i$ to the other modal systems may be illustrated as below. We do not include Hintikka's knowledge system [11] in the following figure. However, we note that it is a special case of K4 with the language so restricted as not to contain $O$ in $S p$. For any set $S,|S|$ will denote its cardinality.

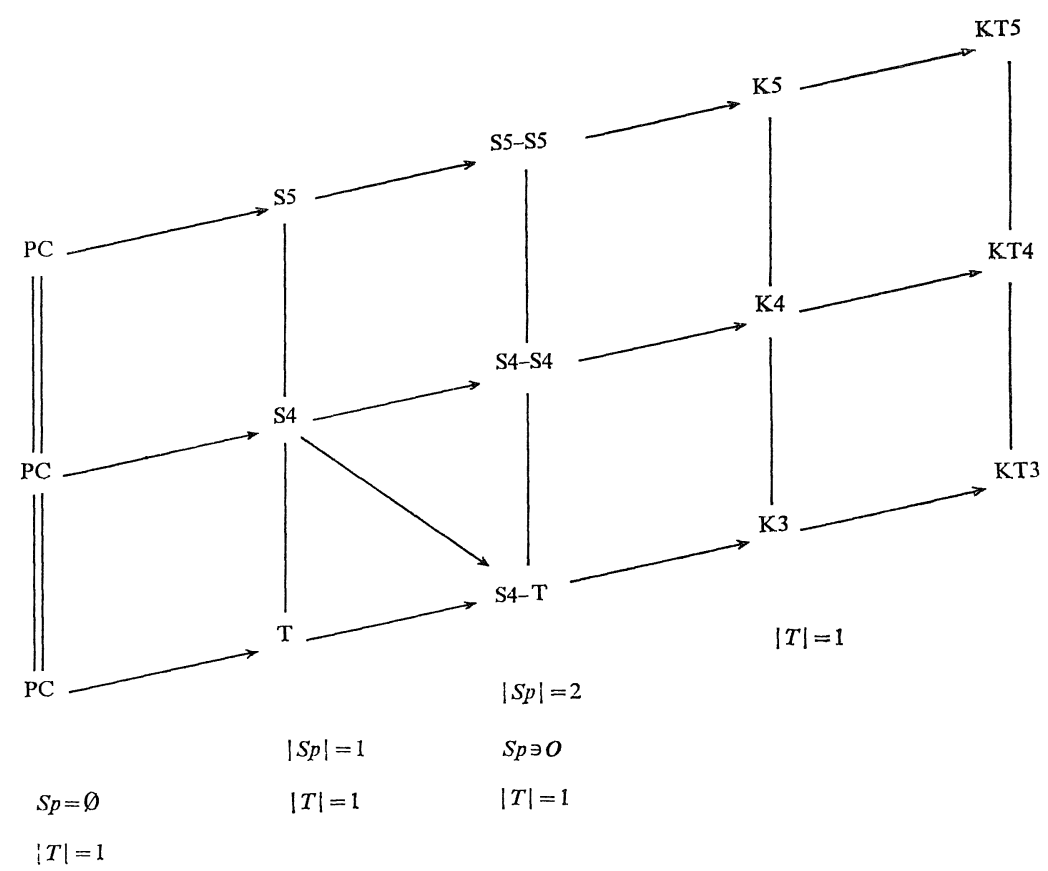

Fig. 1.1. Relation of KTi to other modal logics

In the above diagram, K3, K4 and K5 are the systems in McCarthy [21], Sato [30], and PC denotes the classical propositional calculus. The restrictions imposed on the language to obtain a desired logical system is shown below the name of the system. Furthermore, an arrow 
$A \rightarrow B$ indicates that $A$ is a subsystem of $B$. For example, the modal system $\mathrm{S} 4$ is obtained from $\mathrm{KT} 4$ by restricting $S p$ and $T$ to be singleton sets. The systems on the same vertical line are arranged according to their deductive power. Thus, for example, anything provable in S4 is provable in S5.

(4) Hayashi [8] has pointed out that $\mathrm{KT} 3+(\mathrm{A} 8)$ is already equivalent to $\mathrm{KT} 5(=\mathrm{KT} 3+(\mathrm{A} 7)+(\mathrm{A} 8))$.

\subsection{Gentzen-type Systems}

We now define Gentzen-type systems $\operatorname{GT} i(i=3,4,5)^{2)}$ which are equivalent to $\mathrm{KT} i$. By a sequent we will mean an element in the set $2^{\text {wff }} \times 2^{\text {wff }}$. Namely, it is a pair of (possibly infinite) sets of wffs. Note that our notion of a sequent differs from the original one due to Gentzen [4] at least in the following points. Gentzen defines a sequent as a finite figure of the form $\alpha_{1}, \ldots, \alpha_{m} \rightarrow \beta_{1}, \ldots, \beta_{n}$ while we define a sequent more abstractly and admits infinite sets of wffs.

In order to match with Gentzen's notation, we will denote a sequent by $\Gamma \rightarrow \Delta$ rather than by $(\Gamma, \Delta)$, where $\Gamma, \Delta \subseteq \mathrm{Wff}$. Like this, subsets of Wff will be denoted by Greek capitals. Furthermore, we will employ the abbreviations such as:

$$
\begin{aligned}
& \Gamma \rightarrow \Delta, \quad \Pi=\Gamma \rightarrow \Delta \cup \Pi, \\
& \alpha, \Gamma, \beta \rightarrow=\{\alpha\} \cup \Gamma \cup\{\beta\} \rightarrow \varnothing .
\end{aligned}
$$

Thus, for example, $\alpha, \beta \rightarrow \gamma, \delta, \gamma, \beta, \alpha \rightarrow \delta, \delta, \gamma$ and $\alpha, \alpha, \beta, \beta \rightarrow \gamma, \delta$ denote the same sequent $(\{\alpha, \beta\},\{\gamma, \delta\})$.

We will also use the following notation:

(1) $\Gamma_{0} \rightarrow \Delta_{0} \subseteq \Gamma \rightarrow \Delta$ iff $\Gamma_{0} \subseteq \Gamma$ and $\Delta_{0} \subseteq \Delta$. (In this case, we say $\Gamma_{0} \rightarrow \Delta_{0}$ is a restriction of $\Gamma \rightarrow \Delta$, or $\Gamma \rightarrow \Delta$ is an extension of $\Gamma_{0} \rightarrow \Delta_{0}$.)

(2) $\Gamma_{0} \Subset \Gamma$ iff $\Gamma_{0} \subseteq \Gamma$ and $\Gamma_{0}$ is finite.

(3) $\Gamma_{0} \rightarrow \Delta_{0} \Subset \Gamma \rightarrow \Delta$ iff $\Gamma_{0} \Subset \Gamma$ and $\Delta_{0} \Subset \Delta$.

2) Our definitions of GTi are motivated by Ohnishi-Matsumoto [24]. 
Now, we give the definition of GT3.

Axioms: $\quad \alpha \rightarrow \alpha$

$\perp \rightarrow$

$$
\begin{aligned}
& \text { Rules: } \frac{\Gamma \rightarrow \Delta}{\Pi, \Gamma \rightarrow \Delta, \Sigma} \quad \text { (extension) } \\
& \frac{\frac{\Gamma \rightarrow \Delta, \alpha \quad \alpha, \Pi \rightarrow \Sigma}{\Gamma, \Pi \rightarrow \Delta, \Sigma} \quad \text { (cut) }}{\frac{\Gamma \rightarrow \Delta, \alpha \quad \beta, \Pi \rightarrow \Sigma}{\alpha \supset \beta, \Gamma, \Pi \rightarrow \Delta, \Sigma} \quad(\supset \rightarrow)} \\
& \frac{\alpha, \Gamma \rightarrow \Delta, \beta}{\Gamma \rightarrow \Delta, \alpha \supset \beta} \quad(\rightarrow \supset) \\
& \frac{\alpha, \Gamma \rightarrow \Delta}{[S t] \alpha, \Gamma \rightarrow \Delta} \quad([S t] \rightarrow) \\
& \frac{\Gamma,[O u] \Pi \rightarrow \alpha}{[S u] \Gamma,[O u] \Pi \rightarrow[S t] \alpha} \quad(\rightarrow u,[S t])_{3}, \text { where } u \leq t
\end{aligned}
$$

In the above, the rules $([S t] \rightarrow)$ and $(\rightarrow u,[S t])_{3}$ are rule schemata, where $S$ is an arbitrary element in $S p$ and $t, u$ are arbitrary elements in $T$. One may apply the rule $(\rightarrow u,[S t])_{3}$ only when $u \leq t$. Also in the above for any $\Gamma \subseteq \mathrm{Wff}, S \in S p$ and $t \in T,[S t] \Gamma$ denotes the set $\{[S t] \alpha \mid$ $\alpha \in \Gamma\}$. The notion of a proof in GT3 is defined similarly as in Gentzen's LK [4]. Note, however, that we allow the sequent $\perp \rightarrow$ as a beginning sequent. We write $\vdash \Gamma \rightarrow \Delta$ (in GT3) if it is provable in GT3.

The following inference rules are easily seen to be admissible in GT3:

$$
\begin{aligned}
& \left.\frac{\Gamma \rightarrow \Delta}{\alpha, \Gamma \rightarrow \Delta} \quad \text { (thinning } \rightarrow\right) \\
& \frac{\Gamma \rightarrow \Delta}{\Gamma \rightarrow \Delta, \alpha} \quad(\rightarrow \text { thinning })
\end{aligned}
$$




$$
\begin{aligned}
& \left.\frac{\alpha, \alpha, \Gamma \rightarrow \Delta}{\alpha, \Gamma \rightarrow \Delta} \quad \text { (contraction } \rightarrow\right) \\
& \frac{\Gamma \rightarrow \Delta, \alpha, \alpha}{\Gamma \rightarrow \Delta, \alpha} \quad(\rightarrow \text { contraction }) \\
& \frac{\Gamma, \alpha, \beta, \Pi \rightarrow \Delta}{\Gamma, \beta, \alpha, \Pi \rightarrow \Delta} \quad \text { (interchange } \rightarrow \text { ) } \\
& \frac{\Gamma \rightarrow \Delta, \alpha, \beta, \Sigma}{\Gamma \rightarrow \Delta, \beta, \alpha, \Sigma} \quad \text { ( } \rightarrow \text { interchange) } \\
& \frac{\Gamma \rightarrow \Delta, \alpha}{\neg \alpha, \Gamma \rightarrow \Delta} \quad(\neg \rightarrow) \\
& \frac{\alpha, \Gamma \rightarrow \Delta}{\Gamma \rightarrow \Delta, \neg \alpha} \quad(\rightarrow \neg) \\
& \frac{\alpha, \Gamma \rightarrow \Delta \quad \beta, \Gamma \rightarrow \Delta}{\alpha \vee \beta, \Gamma \rightarrow \Delta} \quad(\vee \rightarrow) \\
& \frac{\Gamma \rightarrow \Delta, \alpha}{\Gamma \rightarrow \Delta, \alpha \vee \beta} \quad \frac{\Gamma \rightarrow \Delta, \beta}{\Gamma \rightarrow \Delta, \alpha \vee \beta} \quad(\rightarrow \vee) \\
& \frac{\alpha, \Gamma \rightarrow \Delta}{\alpha \wedge \beta, \Gamma \rightarrow \Delta} \quad \frac{\beta, \Gamma \rightarrow \Delta}{\alpha \wedge \beta, \Gamma \rightarrow \Delta} \quad(\wedge \rightarrow) \\
& \frac{\Gamma \rightarrow \Delta, \alpha \quad \Gamma \rightarrow \Delta, \beta}{\Gamma \rightarrow \Delta, \alpha \wedge \beta}(\rightarrow \wedge)
\end{aligned}
$$

For example, the following proof figure shows that $(\vee \rightarrow)$ is admissible in GT3:

$$
\frac{\frac{\alpha, \Gamma \rightarrow \Delta}{\alpha, \Gamma \rightarrow \Delta, \perp} \quad \text { (extension) }}{\frac{\Gamma \rightarrow \Delta, \alpha \supset \perp}{(\alpha \supset \perp) \supset \beta, \Gamma \rightarrow \Delta}(\rightarrow \supset) \quad \beta, \Gamma \rightarrow \Delta} \quad(\supset \rightarrow)
$$


This means that, in spite of the difference in the definition of a sequent, every proof figure in (propositional) LK may itself be considered as one in GT3.

Now, GT4 is obtained from GT3 by replacing the rule $(\rightarrow u,[S t])_{3}$ by the following:

$$
\frac{[\mathrm{Su}] \Gamma,[\mathrm{Ou}] \Pi \rightarrow \alpha}{[\mathrm{Su}] \Gamma,[\mathrm{Ou}] \Pi \rightarrow[\mathrm{St}] \alpha} \quad(\rightarrow u,[S t])_{4}, \text { where } u \leq t
$$

GT5 is obtained from GT4 by changing the rule $(\rightarrow u,[S t])_{4}$ to:

$$
\frac{[\mathrm{Su}] \Gamma,[\mathrm{Ou}] \Pi \rightarrow[\mathrm{Ou}] \Sigma,[\mathrm{Su}] \Delta, \alpha}{[\mathrm{Su}] \Gamma,[\mathrm{Ou}] \Pi \rightarrow[\mathrm{Ou}] \Sigma,[\mathrm{Su}] \Delta,[\mathrm{St}] \alpha}(\rightarrow u,[S t])_{5},
$$

where, $u \leq t$

\subsection{Some Metatheorems}

Let us call a sequent $\Gamma \rightarrow \Delta$ finite if both $\Gamma$ and $\Delta$ are finite. Then the following lemma is easily obtained.

Lemma 1.3. If a finite sequent $\Gamma \rightarrow \Delta$ is provable (in GTi) then each sequent occurring in any proof of $\Gamma \rightarrow \Delta$ is finite.

Theorem 1.4. If $\vdash \Gamma \rightarrow \Delta$ (in $\mathrm{KT} i)$ then there exist some $\Gamma_{0} \Subset \Gamma$ and $\Delta_{0} \Subset \Delta$ such that $\vdash \Gamma_{0} \rightarrow \Delta_{0}$ (in $\left.\mathrm{KT} i\right)$.

Proof. By induction on the number $n$ of sequents occurring in the proof of $\Gamma \rightarrow \Delta$.

( $n=1)$ : Since $\Gamma \rightarrow \Delta$ is a beginning sequent, $\Gamma \rightarrow \Delta$ itself is finite.

$(n>1)$ : We consider the case that the last (i.e., downmost) inference is $(\supset \rightarrow)$. The proof then is of the form:

$$
\begin{aligned}
& \begin{array}{ll}
\because \because \bullet^{\circ} & \ddots \\
\Pi \rightarrow \Sigma, \alpha & \beta, \Phi \rightarrow \Psi
\end{array} \\
& \frac{\Pi \rightarrow \Sigma, \alpha \quad \beta, \Phi \rightarrow \Psi}{\alpha \supset \beta, \Pi, \Phi \rightarrow \Sigma, \Psi} \quad(\supset \rightarrow)
\end{aligned}
$$


By induction hypothesis, we have finite $\Pi_{0}, \Sigma_{0}, \Phi_{0}, \Psi_{0}$ such that

$$
\begin{array}{ll}
\ddots \ddots \vdots & \\
\frac{\Pi_{0} \rightarrow \Sigma_{0}}{\Pi \rightarrow \Sigma, \alpha} & \text { (extension) and } \\
\ddots \ddots \bullet^{\circ} & \\
\frac{\Phi_{0} \rightarrow \Psi_{0}}{\beta, \Phi \rightarrow \Psi} & \text { (extension) }
\end{array}
$$

Then we construct the following proof figure.

$$
\begin{array}{cc}
\because \because \vdots \bullet^{\bullet} & \ddots \vdots \vdots \bullet^{\circ} \\
\frac{\Pi_{0} \rightarrow \Sigma_{0}}{\Pi_{0} \rightarrow \Sigma_{0}-\alpha, \alpha} & \frac{\Phi_{0} \rightarrow \Psi_{0}}{\beta, \Phi_{0}-\beta \rightarrow \Psi_{0}} \\
\hline \frac{\alpha \supset \beta, \Pi_{0}, \Phi_{0}-\beta \rightarrow \Sigma_{0}-\alpha, \Psi_{0}}{\alpha \supset \beta, \Pi, \Phi \rightarrow \Sigma, \Psi}
\end{array}
$$

We see that $\alpha \supset \beta, \Pi_{0}, \Phi_{0}-\beta \rightarrow \Sigma_{0}-\alpha, \Psi_{0}$ serves as the desired sequent. Other cases may be dealt with similarly.

Theorem 1.5. For any $\alpha \in \mathrm{Wff}, \vdash \alpha$ (in KTi) if and only if $\vdash \rightarrow \alpha$ (in GTi).

Proof. We only prove the case $i=5$.

Proof of only if part: Left to the reader.

Proof of if part: We prove that if a finite sequent $\Gamma \rightarrow \Delta$ is provable in GT5 then $T \wedge \alpha_{1} \wedge \cdots \wedge \alpha_{m} \supset \beta_{1} \vee \cdots \vee \beta_{n} \vee \perp$ is provable in KT5, where $\alpha_{1}, \ldots, \alpha_{m}\left(\beta_{1}, \ldots, \beta_{n}\right)$ is any enumeration of $\Gamma(\Delta$, resp. $)$ with possible repetitions. First note that $\left(T \wedge \alpha_{1} \wedge \cdots \wedge \alpha_{m} \supset \beta_{1} \vee \cdots \vee \beta_{n} \vee \perp\right) \supset\left(T \wedge \alpha_{1}^{\prime} \wedge \cdots \wedge\right.$ $\left.\alpha_{p}^{\prime} \supset \beta_{1}^{\prime} \vee \cdots \vee \beta_{q}^{\prime} \vee \perp\right)$ is provable in KT5 if $\left\{\alpha_{1}, \ldots, \alpha_{m}\right\}=\left\{\alpha_{1}^{\prime}, \ldots, \alpha_{p}^{\prime}\right\}$ and $\left\{\beta_{1}, \ldots, \beta_{n}\right\}=\left\{\beta_{1}^{\prime}, \ldots, \beta_{q}^{\prime}\right\}$. The proof is carried out by induction on the construction of the proof. We only deal with the rules $([S t] \rightarrow)$ and $(\rightarrow u,[S t])_{5}$. Suppose $[S t] \alpha, \alpha_{1}, \ldots, \alpha_{m} \rightarrow \beta_{1}, \ldots, \beta_{n}$ is obtained from $\alpha, \alpha_{1}, \ldots, \alpha_{m} \rightarrow \beta_{1}, \ldots, \beta_{n}$ by an application of $([S t] \rightarrow)$. Then by induction hypothesis, $\vdash\left(T \wedge \alpha \wedge \alpha_{1} \wedge \cdots \wedge \alpha_{m}\right) \supset\left(\beta_{1} \vee \cdots \vee \beta_{n} \vee \perp\right)$ (in KT5). Since 
$\vdash[S t] \alpha \supset \alpha$, we have $\vdash\left(T \wedge[S t] \alpha \wedge \alpha_{1} \wedge \cdots \wedge \alpha_{m}\right) \supset\left(T \wedge \alpha \wedge \alpha_{1} \wedge \cdots \wedge \alpha_{m}\right)$. Hence, $\quad \vdash\left(T \wedge[S t] \alpha \wedge \alpha_{1} \wedge \cdots \wedge \alpha_{m}\right) \supset\left(\beta_{1} \vee \cdots \vee \beta_{n} \vee \perp\right)$. Next, suppose $[S t] \alpha_{1}, \ldots,[S t] \alpha_{m},[O t] \gamma_{1}, \ldots,[O t] \gamma_{p} \rightarrow[O t] \delta_{1}, \ldots,[O t] \delta_{q},[S t] \beta_{1}, \ldots,[S t] \beta_{n},[S u] \alpha$ is obtained from $[S t] \alpha_{1}, \ldots,[S t] \alpha_{m},[O t] \gamma_{1}, \ldots,[O t] \gamma_{p} \rightarrow[O t] \delta_{1}, \ldots,[O t] \delta_{q}$, $[S t] \beta_{1}, \ldots,[S t] \beta_{n}, \alpha$ by an application of $(\rightarrow u,[S t])_{5}$. By induction hypothesis,

$$
\begin{gathered}
\vdash\left(\top \wedge[S t] \alpha_{1} \wedge \cdots \wedge[S t] \alpha_{m} \wedge[O t] \gamma_{1} \wedge \cdots \wedge[O t] \gamma_{p}\right) \supset \\
\quad\left([O t] \delta_{1} \vee \cdots \vee[O t] \delta_{q} \vee[S t] \beta_{1} \vee \cdots \vee[S t] \beta_{n} \vee \perp\right)
\end{gathered}
$$

Noting that

$$
\vdash[S t](\alpha \supset \beta) \supset([S t] \alpha \supset[S t] \beta)
$$

and

$$
\vdash[S t] \sigma_{1} \wedge \cdots \wedge[S t] \sigma_{k} \supset[S t]\left(\sigma_{1} \wedge \cdots \wedge \sigma_{k}\right)
$$

we have from (1), by necessitation and above,

$$
\begin{aligned}
\vdash \top & \wedge[S t][S t] \alpha_{1} \wedge \cdots \wedge[S t][S t] \alpha_{m} \wedge[S t][O t] \gamma_{1} \wedge \cdots \wedge \\
& {[S t][O t] \gamma_{p} \wedge[S t] \neg[O t] \delta_{1} \wedge \cdots \wedge[S t] \neg[O t] \delta_{q} \wedge } \\
& {[S t] \neg[S t] \beta_{1} \wedge \cdots \wedge[S t] \neg[S t] \beta_{n} \supset[S t] \alpha . }
\end{aligned}
$$

Since

$$
\begin{aligned}
& \vdash[S t] \alpha_{i} \supset[S t][S t] \alpha_{i}, \\
& \vdash[O t] \gamma_{i} \supset[S t][O t] \gamma_{i}, \\
& \vdash \neg[O t] \delta_{i} \supset[S t] \neg[O t] \delta_{i}
\end{aligned}
$$

and

$$
\vdash \neg[S t] \beta_{i} \supset[S t] \neg[S t] \beta_{i}
$$

we have

$$
\vdash \top \wedge[S t] \alpha_{1} \wedge \cdots \wedge[S t] \alpha_{m} \wedge[O t] \gamma_{1} \wedge \cdots \wedge[O t] \gamma_{p} \supset
$$




$$
[O t] \delta_{1} \vee \cdots \vee[O t] \delta_{q} \vee[S t] \beta_{1} \vee \cdots \vee[S t] \beta_{m} \vee[S t] \alpha \vee \perp
$$

which was to be proved.

Corollary 1.6. Let $\Gamma \subseteq \mathrm{Wff}$ and $\alpha \in \mathrm{Wff}$. Then $\Gamma \vdash \alpha$ (in KTi) if and only if $\vdash \Gamma \rightarrow \alpha($ in GTi).

Proof. Only if part: By definition, $\Gamma \vdash \alpha$ implies the existence of some $\beta_{1}, \ldots, \beta_{n} \in \Gamma$ such that $\vdash \beta_{1} \supset\left(\beta_{2} \supset \cdots\left(\beta_{n} \supset \alpha\right) \cdots\right)$. Hence $\vdash \beta_{1}, \ldots, \beta_{n}$ $\rightarrow \alpha$. By (extension) we have $\vdash \Gamma \rightarrow \alpha$.

If part: By Lemma 1.4, there exist some $\beta_{1}, \ldots, \beta_{n}$ such that $\vdash \beta_{1}, \ldots, \beta_{n}$ $\rightarrow \alpha$. Hence $\vdash \rightarrow \beta_{1} \supset\left(\beta_{2} \supset \cdots\left(\beta_{n} \supset \alpha\right) \cdots\right)$. By Theorem 1.5, $\vdash \beta_{1} \supset\left(\beta_{2} \cdots\right.$ $\left.\supset\left(\beta_{n} \supset \alpha\right) \cdots\right)$. This means $\Gamma \vdash \alpha$.

For any $\Gamma \subseteq \mathrm{Wff}$, we let $\neg \Gamma=\{\neg \alpha \mid \alpha \in \Gamma\}$. The following lemma is easy to ascertain.

\section{Lemma 1.7.}

$$
\begin{array}{cc}
\vdash \Gamma \rightarrow \Delta & \text { (in GTi) } \\
\text { iff } \vdash \rightarrow \Delta, \neg \Gamma & \text { (in GTi) } \\
\text { iff } \vdash \neg \Delta, \Gamma \rightarrow & \text { (in GTi). }
\end{array}
$$

\section{Chapter 2}

\section{Topology on $2^{\text {wff }}$}

Scott [33] has introduced $P \omega$ as a model for type-free lamda calculus. It is also designed as a universal domain of computation. In this chapter we introduce a topology on $2^{\text {wff }}$ which is homeomorphic to $P \omega$ topology. We then show that several syntactical properties of our logical systems may be conveniently expressed in terms of topological languages. The result in this chapter tells us the naturalness of considering infinite sequents. This chapter is independent of the remaining chapters. 


\subsection{Definition of Topology}

We now define a topology on $2^{\mathrm{wff}}$. For any finite $\Gamma \subseteq \mathrm{Wff}$, we put $U_{\Gamma}=\left\{\Delta \in 2^{\mathrm{wff}} \mid \Gamma \subseteq \Delta\right\} . \quad\left\{U_{\Gamma} \mid \Gamma\right.$ : finite $\}$ forms a basis of open sets. I. e., $X \subseteq 2^{\text {wff }}$ is, by definition, open if and only if it may be written as a union of some $U_{\Gamma}$ 's. Since Wff is a denumerable set it is clear that under this topology $2^{\text {wff }}$ is homeomorphic to Scott's $P \omega$. Following Scott, we write $T$ for Wff and $\perp$ for the empty set $\emptyset$, since these are top and bottom elements of the Boolean lattice $2^{\text {wff }}$ (under the inclusionship ( $\subseteq$ ) ordering). We define several functions on $2^{\text {wff }}$ as follows.

$$
\text { not: } 2^{\text {wff }} \longrightarrow 2^{\text {wff }}
$$

is defined by:

$$
\operatorname{not}(\Gamma)=\neg \Gamma
$$

$$
\text { isinconsistent }_{i}{ }^{3)}: 2^{\text {wff }} \longrightarrow 2^{\text {wff }}
$$

is defined by:

$$
\text { isinconsistent }_{i}(\Gamma)= \begin{cases}\top & (\text { if } \Gamma \vdash \perp \quad(\text { in } \mathrm{KT} i)) \\ \perp & \text { (otherwise) }\end{cases}
$$

where $i=3,4,5$.

$$
\text { istheorem }{ }_{i}: 2^{\mathrm{wff}} \longrightarrow 2^{\mathrm{wff}}
$$

is defined by:

$$
\text { istheorem }_{i}(\Gamma)= \begin{cases}\top & \text { (if } \vdash \alpha_{1} \vee \cdots \vee \alpha_{n} \text { (in } \mathrm{KTT} i \text { ) for some }\left\{\alpha_{1}, \ldots\right. \\ \perp & (\text { otherwise) }\end{cases}
$$

$$
\mathrm{DC}_{i}: 2^{\mathrm{Wff}} \longrightarrow 2^{\mathrm{wff}} \quad \text { (deductive closure) }
$$

is defined by:

$$
\operatorname{DC}_{i}(\Gamma)=\bar{\Gamma}=\{\alpha \mid \Gamma \vdash \alpha \quad(\text { in } \mathrm{KT} i)\}
$$

3) We will abbreviate this to isincons, . 
is defined by:

$$
\text { isprovable }_{i}(\Gamma \rightarrow \Delta)= \begin{cases}T & \text { (if GT } i \vdash \Gamma \rightarrow \Delta) \\ \perp & \text { (otherwise) }\end{cases}
$$

(6)

$$
\text { left: } 2^{\text {Wff }} \times 2^{\text {Wff }} \longrightarrow 2^{\text {wff }}
$$

is defined by:

$$
\operatorname{left}(\Gamma \rightarrow \Delta)=\neg \Delta \cup \Gamma .
$$

(7)

$$
\text { right: } 2^{\text {wff }} \times 2^{\text {wff }} \longrightarrow 2^{\text {wff }}
$$

is defined by:

$$
\operatorname{right}(\Gamma \rightarrow \Delta)=\Delta \cup \neg \Gamma .
$$

\subsection{Topological Characterization of Syntactical Properties}

$2^{\text {wff }}$, with the above topology, is a continuous lattice in the sense of Scott [32], and so is $2^{\mathrm{wff}} \times 2^{\mathrm{wff}}$ with product topology. Then the functions defined in 2.1 are all continuous functions. More precisely, we have the following:

Theorem 2.1. The following diagrams are commutative in the category of continuous lattices with continuous maps.

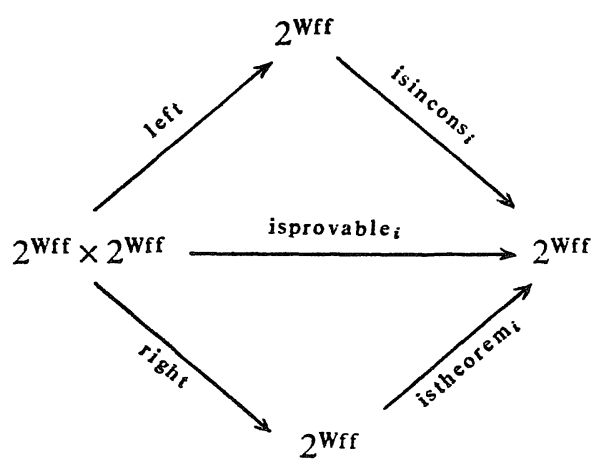



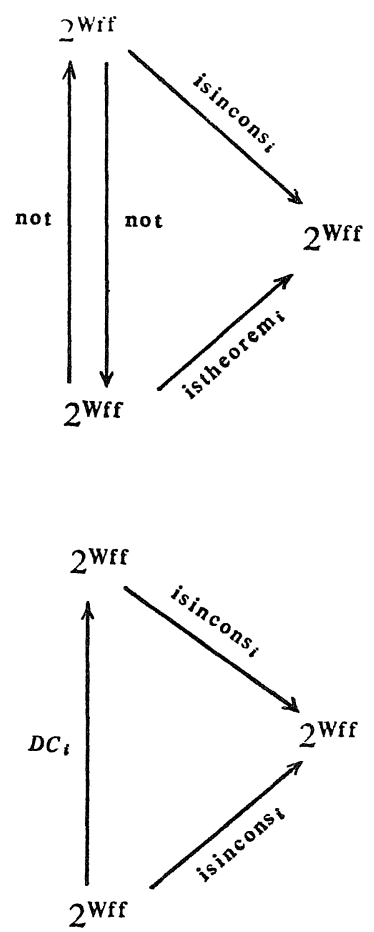

Proof. Commutativity follows from results in 1.6. Continuity is also immediate. For example,

$$
\begin{aligned}
\operatorname{isprovable}_{i}(\Gamma \rightarrow \Delta) & =\bigcup\left\{\operatorname{isprovable}_{i}\left(\Gamma_{0} \rightarrow \Delta\right) \mid \Gamma_{0} \Subset \Gamma\right\} \\
& =\bigcup\left\{\operatorname{isprovable}_{i}\left(\Gamma \rightarrow \Delta_{0}\right) \mid \Delta_{0} \Subset \Delta\right\}
\end{aligned}
$$

by Lemma 1.4. Then by definition in Scott [33], we see isprovable $e_{i}$ is continuous.

The following result is also straightforward. For the definition of retracts and the least fixed point operator $Y$, we refer to Scott [33].

\section{Theorem 2.2.}

(1) istheorem ${ }_{\imath}$, isinconsistent $i$, and $\mathrm{DC}_{i}$ are retracts.

(2) $Y\left(\mathrm{DC}_{i}\right)$ is equal to the set of theorems in $\mathrm{KT} i$.

Remark. Theorem 1.4 is equivalent to the continuity of isprovable ${ }_{i}$. 


\section{Chapter 3}

\section{Kripke-type Semantics}

\subsection{Definition of Kripke-type Models}

Let $W$ be any nonvoid set (of possible worlds). A model $M$ on $W$ is a triple

$$
<W ; r, v>
$$

where

$$
r: S p \times T \longrightarrow 2^{W \times W}
$$

and

$$
v: \operatorname{Pr} \cup\{\perp\} \longrightarrow 2^{W}
$$

Given any model $M$, we define a relation $\vDash \subseteq W \times$ Wff as follows:

(E1) If $\alpha \in \operatorname{Pr} \cup\{\perp\}$ then $w \vDash \alpha$ iff $w \in v(\alpha)$

(E2) If $\alpha=\beta \supset \gamma$ then $w \vDash \alpha$ iff not $w \vDash \beta$ or $w \vDash \gamma$

(E3) If $\alpha=[S t] \beta$ then $w \vDash \alpha$ iff for all $w^{\prime} \in W$ such that $\left(w, w^{\prime}\right) \in r(S$, $t), w^{\prime} \vDash \alpha$

We will write " $w \models \alpha$ (in $M$ )" if we wish to make $M$ explicit. An informal meaning of (E3) is that $[S t] \alpha$ is true in $w$ if and only if $\alpha$ is true in any world accessible to $S$ at time $t$ from $w$. A formula $\alpha$ is said to be valid in $\mathbf{M}$, denoted by $M \models \alpha$, if $w \models \alpha$ for all $w \in M$. (By $w \in M$, we of course mean $w \in W$.) We will write $w \stackrel{s t}{\longrightarrow} w^{\prime}$ instead of $\left(w, w^{\prime}\right) \in r(S, t)$ when $r$ is understood. Furthermore, we will employ the following notations:

$$
\begin{aligned}
& w \vDash \Gamma \text { (read " } w \text { realizes } \Gamma \text { ") iff } w \models \alpha \text { for all } \alpha \in \Gamma \\
& w=\alpha \text { iff not } w \models \alpha \\
& w=\mid \Gamma \text { iff } w=\mid \alpha \text { for all } \alpha \in \Gamma \\
& w=\mid \Gamma \rightarrow \Delta \text { (read " } w \text { realizes } \Gamma \rightarrow \Delta \text { ") iff } w \models \Gamma \text { and } w=\Delta \\
& w \vDash \Gamma \rightarrow \Delta \text { iff not } w=\mid \Gamma \rightarrow \Delta
\end{aligned}
$$


$M \models \Gamma \rightarrow \Delta$ iff $w \models \Gamma \rightarrow \Delta$ for all $w \in M$

A model $M$ is a KT3-model if

(M1) $r(\perp)=\varnothing$

(M2) $r(O, t) \supseteq r(S, t)$ for any $S \in S p$ and $t \in T$

(M3) $r(S, u) \supseteq r(S, t)$ for any $S \in S p$ and $u, t \in T$ such that $u \leq t$

(M4) $r(S, t)$ is a reflexive relation for any $S \in S p$ and $t \in T$

(M5) $r(O, t)$ is a transitive relation for any $t \in T$

A model $M$ is a KT4-model if it satisfies (M1)-(M3) and

(M6) $r(S, t)$ is a reflexive and transitive relation for any $S \in S p$ and $t \in T$

A model $M$ is a KT5-model if it satisfies (M1)-(M3) and

(M7) $r(S, t)$ is an equivalence relation for any $S \in S p$ and $t \in T$

\subsection{Soundness of $\mathrm{KT}$ Ti-models}

We now wish to show that each formula provable in $\mathrm{KT} i$ is valid in any $\mathrm{KT} i$-model. First we prepare some terminology. We say $\Gamma \rightarrow \Delta$ is $i$-provable (i-consistent, resp.) if it is provable (unprovable, resp.) in GTi. We say $\Gamma \rightarrow \Delta$ is i-realizable if there exists some KTi-model $M$ and $w \in M$ such that $w=\mid \Gamma \rightarrow \Delta . \quad \Gamma \rightarrow \Delta$ is said to be $i$-valid if it is not $i$-realizable.

Theorem 3.1 (Soundness Theorem). Any i-provable sequent is i-valid.

Proof. The proof is by induction on the construction of a proof of the given sequent. That any beginning sequent is $i$-valid is immediate from the definition. As for the inference rules, we only treat $(\rightarrow u$, $[S t])_{5}$ of GT5, since other cases are either similar or easier. So, consider:

$$
\frac{[\mathrm{Su}] \Gamma,[\mathrm{Ou}] \Pi \rightarrow[\mathrm{O} u] \Sigma,[\mathrm{Su}] \Delta, \alpha}{[\mathrm{Su}] \Gamma,[\mathrm{Ou}] \Pi \rightarrow[\mathrm{Ou}] \Sigma,[\mathrm{Su}] \Delta,[\mathrm{St}] \alpha,}
$$


where $u \leq t$.

By induction hypothesis, the upper sequent is 5-valid. Suppose, for the sake of contradiction, that the lower sequent is not 5-valid. Then there exist some KT5-model $M$ and $w \in M$ such that

$$
w=[S u] \Gamma,[O u] \Pi \rightarrow[O u] \Sigma,[S u] \Delta,[S t] \alpha .
$$

This implies $w=1[S t] \alpha$. Hence, for some $w^{\prime}$ such that $w \stackrel{S t}{\longrightarrow} w^{\prime}$,

$$
w^{\prime}=\alpha
$$

holds. Since $u \leq t$, we have

$$
w \stackrel{S u}{\longrightarrow} w^{\prime}
$$

by (M3). Then, we have

$$
w \stackrel{O_{u}}{\longrightarrow} w^{\prime}
$$

by (M2). Let $\beta \in \Gamma$ and take any $w^{\prime \prime}$ such that $w^{\prime} \stackrel{S u}{\longrightarrow} w^{\prime \prime}$. Since $r(S, u)$ is transitive by (M7), we have $w \stackrel{S_{u}}{\longrightarrow} w^{\prime \prime}$. Since $w \vDash[S u] \beta$, we have $w^{\prime \prime} \vDash \beta$. This means $w^{\prime} \vDash[S u] \beta$ by (E3). Hence

$$
w^{\prime} \vDash[S u] \Gamma .
$$

Next, take any $\beta$ in $\Delta$. Then, since $w=[S u] \beta$ there exists some $w^{\prime \prime \prime}$ such that

$$
w \stackrel{S u}{\longrightarrow} w^{\prime \prime \prime} .
$$

Since $r(S, u)$ is an equivalence relation we have $w^{\prime} \stackrel{S u}{\longrightarrow} w^{\prime \prime \prime}$ from (2) and (5). Hence, $w^{\prime}=[S u] \beta$ by (E3), so that

$$
w^{\prime}=[S u] \Delta .
$$

From (3) we obtain, similarly as above,

$$
\begin{aligned}
& w^{\prime} \vDash[O u] \Pi, \\
& w^{\prime}=[O u] \Sigma .
\end{aligned}
$$

(1), (4), (6), (7) and (8) means 


$$
w^{\prime}=[S u] \Gamma,[O u] \Pi \rightarrow[O u] \Sigma,[S u] \Delta, \alpha .
$$

This is a contradiction.

Corollary 3.2. If $\vdash \alpha$ (in KTi) then $M \vDash \alpha$ for any KTi-model $M$.

Corollary 3.3 (Consistency of $\mathrm{KT} i$ and GTi). The empty sequent $\rightarrow$ is not provable in GTi.

\subsection{Completeness of KTi-models}

We begin by a syntactical result, which is a kind of Lindenbaum's Lemma.

Lemma 3.4. Let be that $\uparrow \Gamma \rightarrow \Delta$ (in GTi) and $\Phi \supseteq \Gamma \cup \Delta$. Then there exist $\tilde{\Gamma}, \tilde{\Delta}$ such that

(i) $\quad \sim \tilde{\Gamma} \rightarrow \tilde{\Delta} \quad($ in $\mathrm{GT} i)$

(ii) $\tilde{\Gamma} \rightarrow \tilde{\Delta} \supseteq \Gamma \rightarrow \Delta$

(iii) $\tilde{\Gamma} \cup \tilde{\Delta}=\Phi$

Proof. Let $\alpha: \mathbf{N}^{+} \rightarrow \Phi$ be a surjection. We write $\alpha_{i}$ for $\alpha(i)$. We define $\Gamma_{n} \rightarrow \Delta_{n}(n \geq 0)$ as follows:

$$
\begin{aligned}
& \Gamma_{0} \rightarrow \Delta_{0}=\Gamma \rightarrow \Delta \\
& \Gamma_{n+1} \rightarrow \Delta_{n+1}= \begin{cases}\Gamma_{n} \rightarrow \Delta_{n}, \alpha_{n+1} & \left(\text { if }-\Gamma_{n} \rightarrow \Delta_{n}, \alpha_{n+1}\right) \\
\alpha_{n+1}, \Gamma_{n} \rightarrow \Delta_{n} & \text { (otherwise) }\end{cases}
\end{aligned}
$$

We show by induction that $x \Gamma_{n} \rightarrow \Delta_{n}(n \geq 0)$. The case $n=0$ is verified by the assumption of the lemma. Consider the case $n=m+1$, and suppose $\vdash \Gamma_{m+1} \rightarrow \Delta_{m+1}$. Then, by the definition of $\Gamma_{m+1} \rightarrow \Delta_{m+1}$, we have $\vdash \Gamma_{m} \rightarrow \Delta_{m}, \alpha_{m+1}$ and $\vdash \alpha_{m+1}, \Gamma_{m} \rightarrow \Delta_{m}$. From these we obtain $\vdash \Gamma_{m} \rightarrow \Delta_{m}$ by (cut), which contradicts the induction hypothesis.

Now we put $\tilde{\Gamma} \rightarrow \tilde{\Delta}=\bigcup_{n=0}^{\infty} \Gamma_{n} \rightarrow \bigcup_{n=0}^{\infty} \Delta_{n}$. Then we have $\tilde{\Gamma} \rightarrow \tilde{\Delta} \supseteq \Gamma \rightarrow \Delta$ and $\tilde{\Gamma} \cup \tilde{\Delta}=\Phi$. What remains to be shown is that $\tilde{\Gamma} \rightarrow \tilde{\Delta}$ is $i$-consistent. Suppose the contrary. Then by Lemma 1.4 , we have $\Gamma^{\prime} \rightarrow \Delta^{\prime} \Subset \tilde{\Gamma} \rightarrow \tilde{\Delta}$ such that $\vdash \Gamma^{\prime} \rightarrow \Delta^{\prime}$. Now, let $N=\max \left\{n(\beta) \mid \beta \in \Gamma^{\prime} \cup \Delta^{\prime}\right\}$, where $n(\beta)$ 
$=\min \left\{i \mid \beta=\alpha_{i}\right\}$. Then we have $\Gamma^{\prime} \cup \Delta^{\prime} \subseteq \Gamma_{N} \cup \Delta_{N}$. We prove $\Gamma^{\prime} \subseteq \Gamma_{N}$. Suppose $\alpha_{i} \in \Gamma^{\prime}$ and $\alpha_{i} \notin \Gamma_{N}$. Then we have $\alpha_{i} \in \tilde{\Gamma}$ and $\alpha_{i} \in \Delta_{N} \subseteq \tilde{\Delta}$. But $\tilde{\Gamma} \cap \tilde{\Delta}=\varnothing$. This proves $\Gamma^{\prime} \subseteq \Gamma_{N}$. Similarly, $\Delta^{\prime} \subseteq \Delta_{N}$. Since $\vdash \Gamma^{\prime} \rightarrow \Delta^{\prime}$, we have $\vdash \Gamma_{N} \rightarrow \Delta_{N}$, which is a contradiction.

A set $\Omega$ of wffs is said to be closed under subformulas if $\perp \in \Omega$ and $\operatorname{Sub}(\alpha) \subseteq \Omega$ for all $\alpha \in \Omega$. Now take any such $\Omega$ and fix it. We say a sequent $\Gamma \rightarrow \Delta$ is $\Omega$, $i$-complete if $\Gamma \rightarrow \Delta$ is $i$-consistent and $\Gamma \cup \Delta=\Omega$. We denote by $C_{i}(\Omega)$ the set of all $\Omega$, $i$-complete sequents. I.e.,

$$
C_{i}(\Omega)=\{\Gamma \rightarrow \Delta \mid \Gamma \cup \Delta=\Omega, \Gamma \rightarrow \Delta \text { is } i \text {-consistent }\}
$$

We observe that $\Gamma \cap \Delta=\emptyset$ since $\Gamma \rightarrow \Delta$ is $i$-consistent. For any $\Gamma \subseteq \mathrm{Wff}$, $S \in S p$ and $t \in T$, we put $\Gamma_{S t}=\{\alpha \mid[S t] \alpha \in \Gamma\}$. We now define the universal model $U(\Omega)=\langle U ; R, V\rangle$ over $\Omega$ as follows. (Since our definition will depend on the logical system $\mathrm{KT} i$, we will call $U(\Omega)$ the $\Omega$, i-universal model when necessary, and will denote it as $U_{i}(\Omega)$.)

(1) $U=C_{i}(\Omega)$

(2) $V(\alpha)=\{\Gamma \rightarrow \Delta \in U \mid \alpha \in \Gamma\}$, where $\alpha \in \operatorname{Pr} \cup\{\perp\}$

(3) Let $w=\Gamma \rightarrow \Delta \in U, w^{\prime}=\Gamma^{\prime} \rightarrow \Delta^{\prime} \in U$.

$(i=3): \quad\left(w, w^{\prime}\right) \in R(S, t)$ iff $\Gamma_{S u} \subseteq \Gamma^{\prime}$ and $\Gamma_{O u} \subseteq \Gamma_{o_{u}}^{\prime}$ for any $u \leq t$.

$(i=4): \quad\left(w, w^{\prime}\right) \in R(S, t)$ iff $\Gamma_{S u} \subseteq \Gamma_{S u}^{\prime}$ and $\Gamma_{O u} \subseteq \Gamma_{o u}^{\prime}$ for any $u \leq t$.

$(i=5): \quad\left(w, w^{\prime}\right) \in R(S, t)$ iff $\Gamma_{S u}=\Gamma_{S u}^{\prime}$ and $\Gamma_{o u}=\Gamma_{o u}^{\prime}$ for any $u \leq t$.

Lemma 3.5. $U_{i}(\Omega)$ is a $\mathrm{KT} i$-model.

Proof. First, since $\perp \in \Omega$ and $\uparrow \rightarrow \perp$ (Corollary 3.3), Lemma 3.4 assures us that $U=C_{i}(\Omega) \neq \emptyset$.

$(i=3)$ :

(M1) Suppose $w=\Gamma \rightarrow \Delta \in V(\perp)$. Then $\perp \in \Gamma$. Since $\vdash \perp \rightarrow$, we have $\vdash \Gamma \rightarrow \Delta$, which is a contradiction. Hence $V(\perp)=\emptyset$.

(M2), (M3) are immediate from the definition of $R$.

(M4) Let $w=\Gamma \rightarrow \Delta \in U$. Suppose $u \leq t$ and take any $\alpha \in \Gamma_{S u}$. Since $[S u] \alpha \in \Gamma$ and $\Omega$ is closed under subformulas, we have $\alpha \in \Gamma \cup \Delta$. Suppose $\alpha \in \Delta$. Then, since $\vdash[S u] \alpha \rightarrow \alpha$, we have $\vdash \Gamma \rightarrow \Delta$, which is a contradiction. Hence $\alpha \in \Gamma$. This proves $\Gamma_{S u} \subseteq \Gamma$. Since $\Gamma_{O u} \subseteq \Gamma_{o u}$, we see $R(S, t)$ 
is reflexive.

(M5) Let $\left(\Gamma \rightarrow \Delta, \Gamma^{\prime} \rightarrow \Delta^{\prime}\right),\left(\Gamma^{\prime} \rightarrow \Delta^{\prime}, \Gamma^{\prime \prime} \rightarrow \Delta^{\prime \prime}\right) \in R(O, t)$. Suppose $u \leq t$. Then since $\Gamma_{O u} \subseteq \Gamma_{O u}^{\prime} \subseteq \Gamma_{O_{u}}^{\prime \prime}$, we have $\Gamma_{O_{u}} \subseteq \Gamma_{O_{u}}^{\prime \prime}$. We can prove $\Gamma_{o_{u}}^{\prime \prime} \subseteq \Gamma^{\prime \prime}$ as in the proof of (M4), whence $\Gamma_{O u} \subseteq \Gamma^{\prime \prime}$. Thus we see $R(O, t)$ is transitive. The cases $(i=4)$ and $(i=5)$ are now easily seen.

The following theorem will play a key role in the subsequent studies.

Theorem 3.6 (Fundamental Theorem of Universal Model). For any $\alpha \in \Omega$ and $w=\Gamma \rightarrow \Delta \in U(\Omega), w \models \alpha$ (in $U(\Omega)$ ) if $\alpha \in \Gamma$ and $w=\alpha$ (in $U(\Omega))$ if $\alpha \in \Delta$.

Proof. By induction on the construction of formulas.

(1) $\alpha \in \operatorname{Pr} \cup\{\perp\}$ : Immediate from the definition of $R$.

(2) $\alpha=\beta \supset \gamma$ : Suppose $\alpha \in \Gamma$. We must show that $w \neq \beta$ or $w \vDash \gamma$. Suppose, by way of contradiction, that $w \vDash \beta$ and $w=\gamma$. Then, by induction hypothesis, we have $\beta \in \Gamma$ and $\gamma \in \Delta$. Since $\vdash \beta, \beta \supset \gamma \rightarrow \gamma$ (in GT $i$ ), we have $\vdash \Gamma \rightarrow \Delta$ (in GTi), a contradiction. Suppose now $\alpha \in \Delta$. We can prove $w \vDash \beta$ and $w=\mid \gamma$, similarly.

(3) $\alpha=[S t] \beta$ : Suppose $\alpha \in \Gamma$ and take any $w^{\prime}=\Gamma^{\prime} \rightarrow \Delta^{\prime}$ such that $w \stackrel{s t}{\longrightarrow}$ $w^{\prime}$. We show $\beta \in \Gamma^{\prime}$. First, we consider the case $i=3$. Since $\beta \in \Gamma_{S t} \subseteq \Gamma^{\prime}$ we have $\beta \in \Gamma^{\prime}$. Next, we treat the case $i=4,5$. We have $\Gamma_{S t} \subseteq \Gamma_{S t}^{\prime} \subseteq \Gamma^{\prime}$ (see the proof of (M4) in Lemma 3.5). Hence $\beta \in \Gamma^{\prime}$. Thus we see $w \vDash[S t] \beta=\alpha$.

Now suppose $\alpha \in \Delta$.

$(i=3)$ : The sequent $\{[S u] \gamma \in \Gamma \mid u \leq t\},\{[O u] \gamma \in \Gamma \mid u \leq t\} \rightarrow[S t] \beta$ is 3consistent, since it is a restriction of $\Gamma \rightarrow \Delta$. By $(\rightarrow u,[S t])_{3}$, we see $\{\gamma \mid[S u] \gamma \in \Gamma, u \leq t\},\{[O u] \gamma \in \Gamma \mid u \leq t\} \rightarrow \beta$ is also 3 -consistent. Since $\Omega$ is closed under subformulas, we can extend this sequent to an $\Omega, 3$-complete sequent $w^{\prime}=\Gamma^{\prime} \rightarrow \Delta^{\prime}$, by Lemma 3.4. Then for any $u \leq t$, we have $\Gamma_{S u} \subseteq \Gamma^{\prime}$ and $\Gamma_{O u} \subseteq \Gamma_{O u}^{\prime}$. Therefore, we have $w^{\prime} \stackrel{s t}{\longrightarrow} w^{\prime}$. Since $\beta \in \Delta^{\prime}$, by induction hypothesis, we have $w^{\prime}=\{$. Hence $w=\mid[S u] \beta=\alpha$.

$(i=4)$ : Similar to the case $(i=3)$.

$(i=5)$ : Since $\{[S u] \gamma \in \Gamma \mid u \leq t\},\{[O u] \gamma \in \Gamma \mid u \leq t\} \rightarrow\{[O u] \gamma \in \Delta \mid u \leq t\}$, $\{[S u] \gamma \in \Delta \mid u \leq t\},[S t] \beta$ is 5 -consistent as a restriction of $\Gamma \rightarrow \Delta$, we see $\{[\mathrm{Su}] \gamma \in \Gamma \mid u \leq t\},\{[\mathrm{Ou}] \gamma \in \Gamma \mid u \leq t\} \rightarrow\{[\mathrm{Ou}] \gamma \in \Delta \mid u \leq t\},\{[\mathrm{Su}] \gamma \in \Delta \mid u \leq t\}, \beta \quad$ is 
also 5-consistent. Take an $\Omega$, 5-complete extension $w^{\prime}=\Gamma^{\prime} \rightarrow \Delta^{\prime}$ of this sequent. Clearly, for any $u \leq t$, we have $\Gamma_{S u} \subseteq \Gamma_{S u}^{\prime}, \Delta_{S u} \subseteq \Delta_{S u}^{\prime}, \Gamma_{o u} \subseteq \Gamma_{o u}^{\prime}$ and $\Delta_{O u} \subseteq \Delta_{O u}^{\prime}$. We have $\Gamma_{S u}=\Gamma_{S u}^{\prime}$ because $\Gamma_{S u} \subseteq \Gamma_{S u}^{\prime}=\Omega_{S u}-\Delta_{S u}^{\prime} \subseteq \Omega_{S u}-$ $\Delta_{S u}=\Gamma_{S u}$. Similarly, we have $\Gamma_{O u}=\Gamma_{O u}^{\prime}$. By virtue of the definition of $R$, we have $w \stackrel{S t}{\longrightarrow} w^{\prime}$. Since $\beta \in \Delta^{\prime}$, we have by induction hypothesis $w^{\prime}=1 \beta$, which proves $w=[S t] \beta=\alpha$.

From this theorem we at once have the following results.

Theorem 3.7 (Generalized Completeness Theorem). Any i-consistent sequent is i-realizable.

Proof. Let an $i$-consistent sequent $\Gamma \rightarrow \Delta$ be given. We put $\Omega$ $=\{\perp\} \cup \cup\{\operatorname{Sub}(\alpha) \mid \alpha \in \Gamma \cup \Delta\}$. We construct the $\Omega$, $i$-universal model $U_{i}(\Omega)$. Then by Lemma 3.4 and Theorem 3.6, there exists $w \in U$ such that $w=\mid \Gamma \rightarrow \Delta$.

Corollary 3.8. (Compactness Theorem). Let $\Gamma \subseteq \mathrm{Wff}$. Then, $\Gamma$ is i-realizable if and only if any $\Gamma_{0} \Subset \Gamma$ is i-realizable.

Theorem 3.9. (Completeness and Decidability Theorem). For any $\alpha \in \mathrm{Wff}, \alpha$ is a theorem of $\mathrm{KT} i$ if and only if $\alpha$ is valid in all $\mathrm{KT} i$ models whose cardinality $\leq 2^{n}$, where $n$ is the cardinality of the finite set $\operatorname{Sub}(\alpha) \cup\{\perp\}$.

Proof. Let $\Omega=\operatorname{Sub}(\alpha) \cup\{\perp\}$. Then the result easily follows from Lemma 3.4 and Theorem 3.6.

Remark. Our definition of universal models differs from that of canonical models due to Lemmon-Scott [18], in the following points. Firstly, we define models relative to $\Omega$, while canonical models are defined only for $\Omega=$ Wff. So that we need not use filtration method due to Segerberg [34] to secure decidability of the systems. Secondly, relational structures are defined differently. The naturalness of universal models will become clear in the next chapter. 


\subsection{Cut-free System for S5}

In this and next $\S$, we give our second proof of completeness. It is based on cut-free formulations of the systems, and in this section we first formulate a cut-free system GS5 which is equivalent to GT5 with the language restricted to $|S p|=|T|=1$. Hence GS5 is a cut-free system for the modal calculus S5. In GS5, a sequent is defined to be an element of the set $2^{\text {wff }} \times 2^{\text {wff }} \times 2^{\text {wff }} \times 2^{\text {wff }}$. Thus a sequent is of the form $(\Gamma, \Pi, \Sigma, \Delta)$. However we denote this as $\Gamma ; \Pi \rightarrow \Sigma ; \Delta$. Further we will denote $\Gamma ; \rightarrow ; \Delta(=(\Gamma, \emptyset, \emptyset, \Delta))$ simply as $\Gamma \rightarrow \Delta$. A sequent of this form will be called proper. Other sequents will be called improper. The idea of considering this kind of sequents is due to Sonobe [36]. Since our language is subject to the condition $|S p|=|T|=1$, we will denote $[S t] \alpha$ as $\square \alpha$. GS5 is defined as follows:

Axioms: $\alpha \rightarrow \alpha$

$\perp \rightarrow$

$$
\begin{array}{ll}
\text { Rules: } & \frac{\Gamma \rightarrow \Delta}{\Gamma^{\prime}, \Gamma \rightarrow \Delta, \Delta^{\prime}} \quad \text { (extension: out) } \\
& \frac{\Gamma ; \Pi \rightarrow \Sigma \quad \text { } \quad \text { (extension: in) }}{\Gamma ; \Pi^{\prime}, \Pi \rightarrow \Sigma, \Sigma^{\prime} ; \Delta} \quad \text { (cut) } \\
& \frac{\Gamma \rightarrow \Delta, \alpha \quad \alpha, \Pi \rightarrow \Sigma}{\Gamma, \Pi \rightarrow \Delta, \Sigma} \quad(\rightarrow \text { exit) } \\
& \left.\frac{\Gamma ; \rightarrow \alpha ; \Delta}{\Gamma ; \rightarrow \square \alpha, \Delta} \quad \text { (enter } \rightarrow\right) \\
& \left.\frac{\Gamma, \square \alpha ; \Pi \rightarrow \Sigma ; \Delta}{\Gamma ; \square \alpha, \Pi \rightarrow \Sigma ; \Delta} \quad \text { ( }\right) \quad \text { enter) }
\end{array}
$$




$$
\begin{aligned}
& \frac{\Gamma \rightarrow \Delta, \alpha, \beta \quad \beta, \Phi \rightarrow \Psi, \alpha \quad \alpha, \beta, \Xi \rightarrow \Lambda}{\alpha \supset \beta, \Gamma, \Phi, \Xi \rightarrow \Delta, \Psi, \Lambda} \quad(\supset \rightarrow: \text { out }) \\
& \frac{\alpha, \Gamma \rightarrow \Delta, \beta}{\Gamma \rightarrow \Delta, \alpha \supset \beta} \quad(\rightarrow \supset: \text { out }) \\
& \frac{\Gamma ; \Pi \rightarrow \Sigma, \alpha, \beta ; \Delta \quad \Gamma ; \beta, \Phi \rightarrow \Psi, \alpha ; \Delta \quad \Gamma ; \alpha, \beta, \Xi \rightarrow \Lambda ; \Delta}{\Gamma ; \alpha \supset \beta, \Pi, \Phi, \Xi \rightarrow \Sigma, \Psi, \Lambda ; \Delta} \\
& (\supset \rightarrow: \text { in }) \\
& \frac{\Gamma ; \alpha, \Pi \rightarrow \Sigma, \beta ; \Delta}{\Gamma ; \Pi \rightarrow \Sigma, \alpha \supset \beta ; \Delta} \quad(\rightarrow \supset: \text { in }) \\
& \frac{\alpha, \Gamma \rightarrow \Delta}{\square \alpha, \Gamma \rightarrow \Delta} \quad(\square \rightarrow: \text { out }) \\
& \frac{\square \Gamma \rightarrow \square \Delta, \alpha}{\square \Gamma \rightarrow \square \Delta, \square \alpha} \quad(\rightarrow \square: \text { out })
\end{aligned}
$$

The following lemma shows the equivalence of GS5 with GT5 (over the language restricted as above).

Lemma 3.10. Let $\Phi \rightarrow \Psi$ be a proper sequent. Then $\vdash \Phi \rightarrow \Psi$ (in GT5) if and only if $\vdash \Phi \rightarrow \Psi$ (in GS5).

Proof. Only if part: We have only to prove that the rule $(\supset \rightarrow)$ in GT5 is admissible in GS5. To see this we construct the following proof figure:

$$
\frac{\frac{\Gamma \rightarrow \Delta, \alpha}{\Gamma \rightarrow \Delta, \alpha, \beta} \frac{\beta, \Pi \rightarrow \Sigma}{\beta, \Pi \rightarrow \Sigma, \alpha} \frac{\beta, \Pi \rightarrow \Sigma}{\alpha, \beta, \Pi \rightarrow \Sigma}}{\alpha \supset \beta, \Gamma, \Pi \rightarrow \Delta, \Sigma} \quad(\supset \rightarrow: \text { out })
$$

If part: Suppose that $\vdash \Phi \rightarrow \Psi$ (in GS5). We note that Lemmas 1.3 and 1.4 hold also for GS5. Then, by Lemma 1.4, there exists $\Phi_{0} \rightarrow \Psi_{0}$ $\Subset \Phi \rightarrow \Psi$ such that $\vdash \Phi_{0} \rightarrow \Psi_{0}$ (in GS5). Let $\boldsymbol{F}$ be a proof figure of $\Phi_{0}$ $\rightarrow \Psi_{0}$. Then by Lemma 1.3 , any sequent occurring in $F$ is finite, where $\Gamma ; \Pi \rightarrow \Sigma ; \Delta$ is finite if so are $\Gamma, \Pi, \Sigma, \Delta$. We convert $\boldsymbol{F}$ to a proof 
figure in GT5 whose end-sequent is $\Phi_{0} \rightarrow \Psi_{0}$. Let $\Gamma ; \Pi \rightarrow \Sigma ; \Delta$ be any improper sequent occurring in $\boldsymbol{F}$. We replace this sequent by the proper sequent $\Gamma \rightarrow \Delta, \square \alpha$, where $\alpha=\left(\top \wedge \pi_{1} \wedge \cdots \wedge \pi_{m}\right) \supset\left(\sigma_{1} \vee \cdots \vee \sigma_{n} \vee \perp\right)\left(\Pi=\left\{\pi_{1}\right.\right.$, $\left.\left.\ldots, \pi_{m}\right\}, \Sigma=\left\{\sigma_{1}, \ldots, \sigma_{n}\right\}\right)$. We do this replacement for all improper sequents in $\boldsymbol{F}$. By this replacement, for example, an application of the rule

$$
(\text { enter } \rightarrow) \quad \frac{\Gamma, \square \alpha ; \Pi \rightarrow \Sigma ; \Delta}{\Gamma ; \square \alpha, \Pi \rightarrow \Sigma ; \Delta}
$$

will become

$$
\frac{\Gamma, \square \alpha \rightarrow \Delta, \square(\pi \supset \sigma)}{\Gamma \rightarrow \Delta, \square(\square \alpha \wedge \pi \supset \sigma),}
$$

where $\pi=\top \wedge \pi_{1} \wedge \cdots \wedge \pi_{m}\left(\Pi=\left\{\pi_{1}, \ldots, \pi_{m}\right\}\right)$ and $\sigma=\sigma_{1} \vee \cdots \vee \sigma_{n} \vee \perp\left(\Sigma=\left\{\sigma_{1}\right.\right.$,

\begin{tabular}{|c|c|c|c|}
\hline & & $\pi \rightarrow \pi$ & $\sigma \rightarrow \sigma$ \\
\hline & $\square \alpha \rightarrow \square \alpha$ & & $\supset \sigma \rightarrow \sigma$ \\
\hline & $\square \alpha \rightarrow \square \alpha, \sigma$ & $\square \alpha \wedge \tau$ & $\supset \sigma \rightarrow \sigma$ \\
\hline & $\square \alpha \wedge \pi \rightarrow \square \alpha, \sigma$ & $\pi \supset \sigma$ & $x \wedge \pi \supset \sigma$ \\
\hline & $\rightarrow \square \alpha, \square \alpha \wedge \pi \supset \sigma$ & $\square(\pi \supset \sigma)-$ & $x \wedge \pi \supset \sigma$ \\
\hline$\Gamma, \square \alpha \rightarrow \Delta, \square(\pi \supset \sigma)$ & $\rightarrow \square \alpha, \square(\square \alpha \wedge \pi \supset \sigma)$ & $\square(\pi \supset \sigma)-$ & $(\square \alpha \wedge \pi \supset \sigma)$ \\
\hline$\Gamma \rightarrow \Delta, \square \alpha \supset \square(\pi \supset \sigma)$ & $\square \alpha \supset \square(\pi \supset c$ & )$\rightarrow \square(\square \alpha \wedge$ & $\sigma)$ \\
\hline
\end{tabular}
$\left.\left.\ldots, \sigma_{n}\right\}\right)$. We change (\#) to the following:

We must also consider the rules other than (enter $\rightarrow$ ). But they can be treated similarly. Therefore we can obtain a proof of $\Phi_{0} \rightarrow \Psi_{0}$ in GT5. From this we obtain a proof of $\Phi \rightarrow \Psi$ in GT5 by (extension).

We say a sequent is strictly provable (in GS5) if it is provable in GS5 without using (cut). A sequent is weakly consistent if it is not strictly provable. By Lemma 3.10 and Theorem 3.1, we have 
Theorem 3.11. If a proper sequent is provable (in GS5) then it is 5-valid.

We now construct a KT5-model $M=\langle W ; r, v\rangle$ which realizes any proper weakly consistent sequent. For any $\alpha \in \mathrm{Wff}$ we put $\operatorname{Sub}_{\square}(\alpha)$ $=\{\square \beta \mid \square \beta \in \operatorname{Sub}(\alpha)\}$. For any finite sequent $\Gamma \rightarrow \Delta$, we say $\Gamma \rightarrow \Delta$ is saturated if:

(i) $\Gamma \rightarrow \Delta$ is weakly consistent

(ii) $\beta \supset \gamma \in \Gamma \cup \Delta$ implies $\{\beta, \gamma\} \subseteq \Gamma \cup \Delta$

(iii) $\square \beta \in \Gamma$ implies $\beta \in \Gamma$

(iv) $\square \beta \in \Delta$ implies $\operatorname{Sub}_{\square}(\beta) \subseteq \Gamma \cup \Delta$

Lemma 3.12. Let a finite sequent $\Gamma \rightarrow \Delta$ be weakly consistent. Then there exists $\tilde{\Gamma} \rightarrow \tilde{\Delta}$ such that $\Gamma \rightarrow \Delta \subseteq \tilde{\Gamma} \rightarrow \tilde{\Delta}$ and $\tilde{\Gamma} \rightarrow \tilde{\Delta}$ is saturated.

Proof. Let $\Omega=\cup\{\operatorname{Sub}(\alpha) \mid \alpha \in \Gamma \cup \Delta\}$. This is a finite set. Let $\mathbb{C}$ $=\{\Pi \rightarrow \Sigma \mid \Pi \rightarrow \Sigma$ is weakly consistent and $\Pi \cup \Sigma \subseteq \Omega\} . \quad C$ is also finite. We construct a sequence $\left\{\Gamma_{n} \rightarrow \Delta_{n}\right\}_{n \geq 0}$ in $C$ as follows. We put $\Gamma_{0} \rightarrow \Delta_{0}$ $=\Gamma \rightarrow \Delta$. By assumption, we have $\Gamma_{0} \rightarrow \Delta_{0} \in C$. Suppose that $\Gamma_{n} \rightarrow \Delta_{n}$ $C$ has been defined. If $\Gamma_{n} \rightarrow \Delta_{n}$ is saturated, we put $\Gamma_{n+1} \rightarrow \Delta_{n+1}=\Gamma_{n} \rightarrow \Delta_{n}$. Suppose otherwise. Then one of (ii)-(iv) in the above definition of a sequent being saturated fails.

(1) Suppose there exists some $\beta \supset \gamma \in \Gamma_{n} \cup \Delta_{n}$ such that $\{\beta, \gamma\} \nsubseteq \Gamma_{n} \cup \Delta_{n}$. Suppose $\beta \supset \gamma \in \Gamma_{n}$. Then by ( $\supset \rightarrow$ : out) we have that one of $\Gamma_{n} \rightarrow \Delta_{n}$, $\beta, \gamma, \gamma, \Gamma_{n} \rightarrow \Delta_{n}, \beta$ or $\beta, \gamma, \Gamma_{n} \rightarrow \Delta_{n}$ is weakly consistent. We define $\Gamma_{n+1}$ $\rightarrow \Delta_{n+1}$ as the first weakly consistent sequent among these three sequents. In case $\beta \supset \hat{\gamma} \in \Delta_{n}$, we put $\Gamma_{n+1} \rightarrow \Delta_{n+1}=\beta, \Gamma_{n} \rightarrow \Delta_{n}, \gamma$.

(2) Suppose that there exists some $\square \beta \in \Gamma_{n}$ such that $\beta \notin \Gamma_{n}$. We put $\Gamma_{n+1} \rightarrow \Delta_{n+1}=\beta, \Gamma_{n} \rightarrow \Delta_{n}$. By $(\square \rightarrow$ : out $)$, we have $\Gamma_{n+1} \rightarrow \Delta_{n+1} \in \mathbb{C}$.

(3) Suppose that there exists some $\square \beta \in \Delta_{n}$ such that $\operatorname{Sub}_{\square}(\beta) \nsubseteq \Gamma_{n} \cup \Delta_{n}$. Let $\square \gamma$ be an element of the set $\operatorname{Sub}_{\square}(\beta)-\left(\Gamma_{n} \cup \Delta_{n}\right)$ with maximal degree, where the degree of a formula is defined to be the number of logical connectives (i.e., $\supset$ and $\square$ ) occurring in it. Let $\square \delta$ be an element of $\Gamma_{n} \cup \Delta_{n}$ such that $\square \gamma \in \operatorname{Sub}(\delta)$ and with minimal degree. The existence of such $\square \delta$ is guaranteed by the fact that $\square \gamma \in \operatorname{Sub}(\beta)$ and $\square \beta \in \Delta_{n}$. Then we have two cases. 
$\square \delta \in \Gamma_{n}$ : Since $\Gamma_{n} \rightarrow \Delta_{n}=\square \delta, \Gamma_{n} \rightarrow \Delta_{n}$ is weakly consistent, so is $\delta, \Gamma_{n} \rightarrow \Delta_{n}$ by $(\square \rightarrow$ : out). Then using ( $\supset \rightarrow$ : out), ( $\rightarrow \supset$ : out) and (extension: out), we see, by reductio ad absurdum, that either $\square \gamma, \Gamma_{n} \rightarrow \Delta_{n}$ or $\Gamma_{n} \rightarrow \Delta_{n}$, $\square \gamma$ is weakly consistent. So, we define $\Gamma_{n+1} \rightarrow \Delta_{n+1}$ as the first weakly consistent sequent of the two.

$\square \delta \in \Delta_{n}$ : Since $\Gamma_{n} \rightarrow \Delta_{n}=\Gamma_{n} \rightarrow \Delta_{n}, \square \delta$ is weakly consistent, so is $\Gamma_{n} ; \rightarrow \delta$; $\Delta_{n}$ by ( $\rightarrow$ exit). Then by ( $\supset \rightarrow:$ in), ( $\rightarrow \supset:$ in) and (extension: in), we see either $\Gamma_{n} ; \square \gamma \rightarrow ; \Delta_{n}$ or $\Gamma_{n} ; \rightarrow \square \gamma ; \Delta_{n}$ is weakly consistent. Since the argument goes similarly, we suppose the first case. Then by (enter $\rightarrow$ ), $\Gamma_{n}, \square \gamma \rightarrow \Delta_{n}$ is weakly consistent. In this case we put $\Gamma_{n+1} \rightarrow \Delta_{n+1}=\Gamma_{n}$, $\square \gamma \rightarrow \Delta_{n}$.

In any of the above three cases, we have $\Gamma_{n+1} \rightarrow \Delta_{n+1} \in \mathbb{C}$ and $\left|\Gamma_{n} \cup \Delta_{n}\right|<\left|\Gamma_{n+1} \cup \Delta_{n+1}\right|$. Therefore, since $C$ is finite, we obtain a saturated $\Gamma_{n} \rightarrow \Delta_{n}$ for some $n$. Putting $\tilde{\Gamma} \rightarrow \tilde{\Delta}=\Gamma_{n} \rightarrow \Delta_{n}$ we have the desired result.

We now define a model $M=\langle W ; r, v>$. Let $W=\{\Gamma \rightarrow \Delta \mid \Gamma \rightarrow \Delta$ is saturated $\}$. $W$ is nonempty since $\rightarrow \perp \in W$. Let $w=\Gamma \rightarrow \Delta, w^{\prime}=\Gamma^{\prime}$ $\rightarrow \Delta^{\prime} \in W$. We define $\left(w, w^{\prime}\right) \in r$ iff $\Gamma_{\square}=\Gamma_{\square}^{\prime}$. (Since $|S p \times T|=1$, we may consider $r: S p \times T \rightarrow 2^{W \times W}$ as an element of $2^{W \times W} . \Gamma_{\square}$ denotes the set $\{\alpha \mid \square \alpha \in \Gamma\}$.) $\quad v: \operatorname{Pr} \cup\{\perp\} \rightarrow 2^{W}$ is defined by that $w=\Gamma \rightarrow \Delta \in v(\alpha)$ iff $\alpha \in \Gamma$. The following lemma is proved similarly as Lemma 3.5 .

\section{Lemma 3.13. $M$ is a KT5-model.}

Just like $U(\Omega), M$ has the following important property:

Theorem 3.14. Let $w=\Gamma \rightarrow \Delta \in M$ and $\alpha \in \Gamma \cup \Delta$. Then $w \vDash \alpha$ (in $M$ ) if $\alpha \in \Gamma$ and $w=\alpha$ if $\alpha \in \Delta$.

Proof. By induction on the construction of formulas. We only consider the case that $\alpha=\square \beta \in \Delta$, since other cases may be handled similarly as in the proof of Theorem 3.6. Now, $\Gamma_{0} \rightarrow \Delta_{0}=\{\square \gamma \mid \square \gamma \in \Gamma\}$ $\rightarrow\{\square \delta \mid \square \delta \in \Delta\}, \square \beta$ is weakly consistent since it is a restriction of $\Gamma \rightarrow \Delta$. By ( $\rightarrow \square$ : out), we see $\Gamma_{1} \rightarrow \Delta_{1}=\{\square \gamma \mid \square \gamma \in \Gamma\} \rightarrow\{\square \delta \mid \square \delta \in \Delta\}, \beta$ is also weakly consistent. By Lemma 3.12, we can extend this sequent to a saturated sequent $w^{\prime}=\Gamma^{\prime} \rightarrow \Delta^{\prime} \in W$. By this construction, it is clear that 
$\Gamma_{\square} \subseteq \Gamma_{\square}^{\prime}$. Suppose $\sigma \in \Gamma_{\square}^{\prime}-\Gamma_{\square}$. Then by inspecting the construction method in Lemma 3.12, we see that $\square \sigma \in \operatorname{Sub}_{\square}\left(\gamma_{1}\right)$ for some $\gamma_{1} \in \Gamma_{1} \cup \Delta_{1}$. Hence, $\square \sigma \in \operatorname{Sub}_{\square}\left(\gamma_{0}\right)$ for some $\gamma_{0} \in \Gamma_{0} \cup \Delta_{0} \subseteq \Gamma \cup \Delta$. (If $\gamma_{1}=\beta$ then let $\gamma_{0}=\square \beta \in \Delta_{0}$, otherwise let $\gamma_{0}=\gamma_{1}$.) Since $\Gamma \rightarrow \Delta$ is saturated, we have $\square \sigma \in \Gamma \cup \Delta$. Since $\sigma \notin \Gamma_{\square}$ we have $\square \sigma \in \Delta$. Hence we have $\square \sigma \in \Gamma^{\prime} \cap \Delta^{\prime}$. This contradicts the consistency of $\Gamma^{\prime} \rightarrow \Delta^{\prime}$. Thus we see $\Gamma_{\square}=\Gamma_{\square}^{\prime}$, so that $\left(w, w^{\prime}\right) \in r$. Now since $\beta \in \Delta^{\prime}$, we have $w^{\prime}=\beta$ by induction hypothesis. Hence we have $w=\exists \square$.

It is now easy to establish:

Theorem 3.15 (Cut-elimination Theorem). If a proper sequent is provable in GS5 then it is strictly provable in GS5.

Proof. By Lemma 1.4 it suffices to consider only finite sequents. We prove the contraposition. Suppose that a finite sequent $\Gamma \rightarrow \Delta$ is not strictly provable. $\Gamma \rightarrow \Delta$ has a saturated extension $\tilde{\Gamma} \rightarrow \tilde{\Delta}$ by Lemma 3.12 . Then $\tilde{\Gamma} \rightarrow \tilde{\Delta}$ is 5 -realizable by Theorem 3.14. Then $\tilde{\Gamma} \rightarrow \tilde{\Delta}$ is not provable by Theorem 3.11. Hence $\Gamma \rightarrow \Delta$ is not provable.

\subsection{Cut-elimination Theorem for GT3 and GT4}

In this section we consider only KT3 and KT4, so that when we refer to $\mathrm{KT} i$ or $\mathrm{GT} i, i$ is always 3 or 4 . If a sequent $\Gamma \rightarrow \Delta$ is provable in GT $i$ without cut, we say $\Gamma \rightarrow \Delta$ is strictly provable. We wish to establish this:

Theorem 3.16 (Cut-elimination Theorem). If a sequent is provable (in GTi) then it is strictly provable.

We prove this by an argument similar to that in 3.3. Let $\Omega \subseteq \mathrm{Wff}$ be closed under subformulas. Let us call a sequent $\Gamma \rightarrow \Delta \Omega$, i-maximal if it is maximal in the set $\{\Pi \rightarrow \Sigma \mid \Pi \rightarrow \Sigma$ is $i$-weakly consistent and $\Pi \cup \Sigma$ $\subseteq \Omega\}$, where a sequent is $i$-weakly consistent if it is not strictly provable in GTi. We can show that if a sequent is $i$-weakly consistent and $\Gamma \cup \Delta \subseteq \Omega$ then it has a maximal extension $\tilde{\Gamma} \rightarrow \tilde{\Delta} \in W_{i}(\Omega)=\{\Pi \rightarrow \Delta \mid \Pi \rightarrow \Sigma$ is $\Omega, i$-maximal\}, by means of Zorn's Lemma and Theorem 1.4. Now, 
we define a model $M_{i}(\Omega)=\left\langle W_{i}(\Omega) ; r, v\right\rangle$, where $r$ and $v$ are defined just as in the definition of $U_{i}(\Omega)$. That $M_{i}(\Omega)$ is a KTi-model is proved similarly as in Lemma 3.5. We now have the following lemma.

Lemma 3.17. Let $w=\Gamma \rightarrow \Delta \in M_{i}(\Omega)$ and $\alpha \in \Gamma \cup \Delta$. Then $w \models \alpha$ (in $M_{i}(\Omega)$ ) if $\alpha \in \Gamma$ and $w=\mid \alpha\left(\right.$ in $\left.M_{i}(\Omega)\right)$ if $\alpha \in \Delta$.

Proof. By induction on the construction of formulas. The base step of $\alpha \in \operatorname{Pr} \cup\{\perp\}$ is trivial.

$\alpha=\beta \supset \gamma$ : Suppose $\alpha \in \Gamma$. Then $\Gamma \rightarrow \Delta, \beta$ or $\gamma, \Gamma \rightarrow \Delta$ is $i$-weakly consistent. By the maximality of $\Gamma \rightarrow \Delta$, we have $\Gamma \rightarrow \Delta, \beta=\Gamma \rightarrow \Delta$ or $\gamma, \Gamma \rightarrow \Delta$ $=\Gamma \rightarrow \Delta$. In any case, we have $w \models \alpha$ by induction hypothesis and definition of $\vDash$. The case $\alpha \in \Delta$ is similar.

$\alpha=[S t] \beta:$ If $\alpha \in \Gamma$, then the result follows similarly as in Theorem 3.6. Suppose $\alpha \in \Delta$.

$(i=3): \quad\{[S u] \gamma \in \Gamma \mid u \leq t\},\{[O u] \gamma \in \Gamma \mid u \leq t\} \rightarrow[S t] \beta$ is $i$-weakly consistent as a restriction of $\Gamma \rightarrow \Delta$. Hence $\{\gamma \mid[S u] \gamma \in \Gamma, u \leq t\},\{[O u] \gamma \in \Gamma \mid u \leq t\}$ $\rightarrow \beta$ is also $i$-weakly consistent. Extend this sequent to $w^{\prime}=\Gamma^{\prime} \rightarrow \Delta^{\prime}$ in $M_{i}(\Omega)$. It is clear that $w \stackrel{S t}{\longrightarrow} w^{\prime}$. Since $\beta \in \Delta^{\prime}$ we have $w^{\prime}=\beta$ by induction hypothesis. Hence $w=\mid \alpha$.

$(i=4)$ : Similar to the case $(i=3)$.

Now we can complete the proof of Theorem 3.16. Suppose $\Gamma \rightarrow \Delta$ is $i$-weakly consistent. Let $\Omega=\{\perp\} \cup \cup\{\operatorname{Sub}(\alpha) \mid \alpha \in \Gamma \cup \Delta\}$. Let $\tilde{\Gamma} \rightarrow \tilde{\Delta}$ $\in M_{i}(\Omega)$ be an extension of $\Gamma \rightarrow \Delta$. Then by Lemma 3.17, $M_{i}(\Omega)=I \Gamma \rightarrow \Delta$. Hence by the Soundness Theorem $3.1, \Gamma \rightarrow \Delta$ is not provable.

\section{Remarks.}

(1) Our method does not work for GT5, because, except for the obvious fact that GT5 is not cut-free, ${ }^{4)}$ if we construct a model $M_{5}(\Omega)$ it does not always give $w^{\prime}$ such that $w \stackrel{S t}{\longrightarrow} w^{\prime}$ and $w^{\prime}=\beta$ for $w$ such that $w$ $=[S t] \beta$. However, as a partial result, we gave a cut-free system for S5 in 3.4 .

(2) By Theorem 3.16, we observe that $M_{i}(\Omega)$ is identical with $U_{i}(\Omega)$ (for $i=3,4)$.

4) For example, the sequent $\rightarrow p,[S t] \neg[S t] p$ (where $p \in P r$ ) is not provable without cut. 
The following theorem will have some significance in Chapter 6 .

Theorem 3.18 (Disjunction property of KT3 and KT4) ${ }^{5}$. Suppose $\vdash\left[S^{1} t_{1}\right] \alpha_{1} \vee \cdots \vee\left[S^{n} t_{n}\right] \alpha_{n}$ (in $\left.\mathrm{KT} i\right)(n \geq 1)$. Then for some $j(1 \leq j \leq n)$ we have $\vdash\left[S^{j} t_{j}\right] \alpha_{j}$ (in $\left.\mathrm{KT} i\right)$, where $i=3$ or 4 .

Proof. Consider a cut-free proof of $\rightarrow\left[S^{1} t_{1}\right] \alpha_{1}, \ldots,\left[S^{n} t_{n}\right] \alpha_{n}$. Let $N=\left|\left\{\left[S^{1} t_{1}\right] \alpha_{1}, \ldots,\left[S^{n} t_{n}\right] \alpha_{n}\right\}\right|$. If $N=1$ then we see that $\vdash \rightarrow\left[S^{1} t_{1}\right] \alpha_{1}$. Let $N>1$. Then the last inference rule must be (extension). Furthermore we may assume without losing generality that the cardinality $|\Delta|$ of the upper sequent $\rightarrow \Delta$ of the last inference is less than $N$. Hence the result follows by induction hypothesis.

In this and the last §, we have seen that GS5, GT3 and GT4 are cut-free. Using this fact, we obtain our second proof of the decidability of these systems as follows.

Theorem 3.19. KT3, KT4 and S5 are decidable.

Proof. Since the proof goes similarly, we only prove the theorem for S5. We first note that any proof figure may be represented as a pair $(\boldsymbol{P}, f)$, where $\boldsymbol{P}=\left(P, \leq_{P}\right)$ is a tree partially ordered by $\leq_{\boldsymbol{P}}$ and $f$ is a function $f: P \rightarrow 2^{\mathrm{wff}} \times 2^{\mathrm{wff}} \times 2^{\mathrm{wff}} \times 2^{\mathrm{wff}}$. More precisely, 1) $P$ is an abstract set such that $|P|$ is equal to the number of sequents occurring in the proof figure, 2) for any node $p \in P, f(p)$ denotes the sequent attached to $p$, and 3) $p \leq_{P} q$ iff $p=q$ or $f(q)$ is above (in the sense of Gentzen $[4,5]) f(p)$ in the proof figure. Suppose a formula $\alpha \in \mathrm{Wff}$ is given. Let $\Omega=\operatorname{Sub}(\alpha)$ and $|\Omega|=n$. Suppose $\alpha$ is provable. Then it has a cut-free proof $(\boldsymbol{P}, f)$. Then we have

$$
\text { Image }(f) \subseteq 2^{\Omega} \times 2^{\Omega} \times 2^{\Omega} \times 2^{\Omega} .
$$

(Subformula property of a cut-free proof!) Furthermore, we may assume without losing generality that $f(p) \neq f(q)$ if $p<_{P} q$. (For, otherwise, we can obtain a smaller proof figure with the same end-sequent $\rightarrow \alpha$.) Thus

5) Using the completeness of KT3, 4-models, Hayashi [9] obtained a model theoretic proof of this theorem by a method due to Kripke [15]. 
we see that any linearly ordered subset $Q$ of $P$ has cardinality less than or equal to $2^{2^{n} \cdot 2^{n} \cdot 2^{n} \cdot 2^{n}}=m$. Since the number of the upper sequents of each inference rule is at most 3 , it follows that

$$
|P| \leq 3^{m}
$$

By (1) and (2), we can construct an algorithm which determines the provability of $\alpha$.

\section{Chapter 4}

\section{Categories of Kripke Models ${ }^{6}$}

\subsection{Definition of $\mathscr{K}_{i}(\Omega)$}

Let $\Omega$ be closed under subformulas. Let us take any $i(3 \leq i \leq 5)$ and fix it. We define the category $\mathscr{K}_{i}(\Omega)$ of KTi-models over $\Omega$ as follows:

(1) Objects $(\mathscr{M})$ are KTi-models.

(2) Let $M, N \in \mathscr{M}$, then $\operatorname{Hom}(M, N)=[M \rightarrow N]$ consists of homomorphisms (from $M$ to $N$ ) as defined below.

(3) Composition of homomorphisms is defined by the usual function composition, i.e., $(f \circ g)(x)$ is defined by $f(g(x))$.

For any $M \in \mathscr{M}$, we define its characteristic function

$$
\chi_{M}: M \longrightarrow U(\Omega)
$$

by $\chi_{M}(w)=\Gamma \rightarrow \Delta$, where $\Gamma=\{\alpha \in \Omega \mid w \models \alpha\}$ and $\Delta=\{\alpha \in \Omega|w=| \alpha\}$. It is clear that $\Gamma \rightarrow \Delta$ is $\Omega$-complete and hence $\chi_{M}$ is well-defined. $(U(\Omega)$ means $U_{i}(\Omega)$ and $\Omega$-complete means $\Omega$, i-complete.) A mapping

$$
h: M \longrightarrow N
$$

is a homomorphism (from $M$ to $N$ ) if the diagram below commutes:

6) Elementary terminology of category theory in this chapter mostly follows Mitchell [23]. 


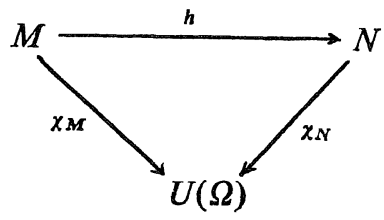

Informally speaking, for $w \in M, \chi_{M}(w)$ denotes the scene (restricted to $\Omega$ ) as seen from $w$. Thus a homomorphism is a mapping which preserves scenes. It is an easy task to verify that $\mathscr{K}_{i}(\Omega)$ defined above is indeed a category. As an example, consider the simplest case of $\Omega=\{\perp\}$. Then any mapping $f: M \rightarrow N$ is a homomorphism.

\subsection{Properties of $\mathscr{K}^{i}(\Omega)$}

First of all, by the Fundamental Theorem of Universal Model, we see that $\chi_{U(\Omega)}: U(\Omega) \rightarrow U(\Omega)$ is the identity mapping $1_{U(\Omega)}$. Hence, for any $M \in \mathscr{M}$, by the following commutative diagram we observe that $\chi_{M}$ itself is a homomorphism.

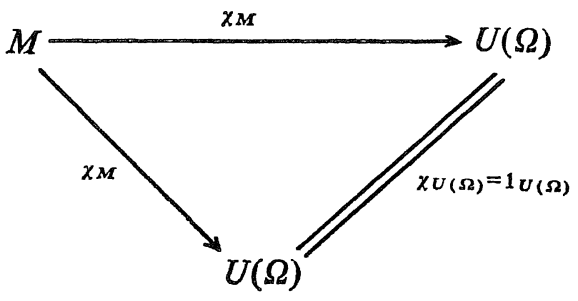

On the other hand, let $h \in[M \rightarrow U(\Omega)]$. Then since the diagram below commutes, we have $h=\chi_{M}$.

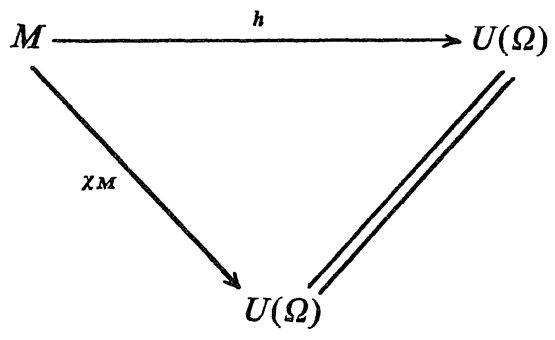

Thus we obtain: 
Theorem 4.1. $U(\Omega)$ is a terminal object ${ }^{7)}$ of $\mathscr{K}(\Omega)$.

We now list up several basic properties of $\mathscr{K}(\Omega)$.

Lemma 4.2. If $f \in[M \rightarrow N]$ is a monomorphism then $f$ is an injection.

Proof. We prove the contraposition. Let $x, y \in M$ be such that $x \neq y$ and $f(x)=f(y)$. Define $g: M \rightarrow N$ by:

$$
g(z)=\left\{\begin{array}{cc}
x & \text { if } \quad z=y \\
y & \text { if } \quad z=x \\
z & \text { otherwise }
\end{array}\right.
$$

Then we have:

$$
\chi_{M}(g(z))= \begin{cases}\chi_{M}(x)=\chi_{N}(f(x))=\chi_{N}(f(y))=\chi_{M}(y) & \text { if } z=y \\ \chi_{M}(y)=\chi_{N}(f(y))=\chi_{N}(f(x))=\chi_{M}(x) & \text { if } z=x \\ \chi_{M}(z) & \text { otherwise }\end{cases}
$$

Hence, $g \in[M \rightarrow N]$. Now, clearly $f \circ g=f \circ 1_{M}$, but $g \neq 1_{M}$. This means $f$ is not a monomorphism.

Lemma 4.3. If $f \in[M \rightarrow N]$ is an epimorphism then $f$ is a surjection.

Proof. We prove the contraposition. Let $N=\langle W ; r, v\rangle$. Let $x \in N$ be such that $x \notin \operatorname{Image}(f)$. Take $y$ such that $y \notin N$. We define a model $\tilde{N}=\langle\tilde{W} ; \tilde{r}, \tilde{v}>$ such that $\tilde{W}=W \cup\{y\}$ as follows: Let $g: \tilde{W}$ $\rightarrow W$ be defined by:

$$
g(z)= \begin{cases}x & \text { if } z=y \\ z & \text { otherwise }\end{cases}
$$

We define $\tilde{r}$ by $\left(w, w^{\prime}\right) \in \tilde{r}(S, t)$ iff $\left(g(w), g\left(w^{\prime}\right)\right) \in r(S, t)$. We define $\tilde{v}$ by

7) Mitchell [23] uses the term null object instead of terminal object. 
$w \in \tilde{v}(p)$ iff $g(w) \in v(p)$. It is easy to verify that $\tilde{N}$ is a KTi-model. We can prove, by induction, that for any $w \in W$ and $\alpha \in \mathrm{Wff}$,

$$
w \vDash \alpha \text { (in } \tilde{N}) \text { iff } g(w) \vDash \alpha(\text { in } N) \text {. }
$$

I.e., $g \in[\tilde{N} \rightarrow N]$. Let $h: N \rightarrow \tilde{N}$ be the inclusion map, and let $h^{\prime}: N \rightarrow \tilde{N}$ be defined by:

$$
h^{\prime}(z)= \begin{cases}y & \text { if } z=x \\ z & \text { otherwise }\end{cases}
$$

We have $g \circ h=g \circ h^{\prime}=1_{N}$.

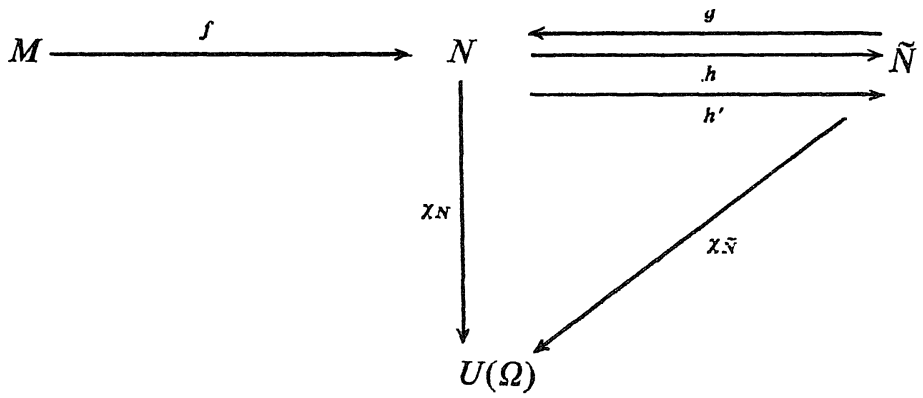

Then we have

$$
\chi_{\bar{N}}(h(z))=\chi_{N}(g(h(z)))=\chi_{N}(z),
$$

so that $h \in[N \rightarrow \tilde{N}]$. Similarly, $h^{\prime} \in[N \rightarrow \tilde{N}]$. Now, clearly, $h \circ f=h^{\prime} \circ f$ but $h \neq h^{\prime}$. This means $h$ is not an epimorphism.

Remark. The reader familiar with the notion of $p$-morphism might have noticed that the homomorphism $g$ in the above proof is a $p$ morphism. By the $p$-morphism theorem [34], every $p$-morphism is a homomorphism (for any $\Omega$ ), but the converse is not valid. In this sense our notion of homomorphism is more general than that of $p$-morphism. Note also that we defined homomorphisms without referring to the relational structure of models.

Lemma 4.4. If $f \in[M \rightarrow N]$ is an epimorphism, $f$ is a retraction.

Proof. By Lemma 4.2, $f$ is onto. Let $g: N \rightarrow M$ be any mapping 
such that $f \circ g=1_{N}$. Let $x \in N$. Then $\chi_{M}(g(x))=\left(\chi_{N^{\circ}} \circ\right)(g(x))=\chi_{N}(f \circ g(x))$ $=\chi_{N}(x)$, i.e., $X_{M} \circ g=\chi_{N}$. Hence $g \in[N \rightarrow M]$. This means $f$ is a retraction.

We cite the following easy lemma from Mitchell [23].

Lemma 4.5. If $f \in[M \rightarrow N]$ is a retraction and also a monomorphism, then it is an isomorphism.

By Lemmas 4.4 and 4.5 , we have

Theorem 4.6. $\mathscr{K}(\Omega)$ is balanced, i.e., every homomorphism which is both a monomorphism and an epimorphism is also an isomorphism.

Lemma 4.7. Let $M \in \mathscr{M}$. Then the following conditions are equivalent:

(i) $\chi_{M}$ is a monomorphism

(ii) For any $N \in \mathscr{M},|[N \rightarrow M]| \leq 1$

(iii) $\operatorname{End}(M)=\left\{1_{M}\right\}$

(iv) $\operatorname{Aut}(M)=\left\{1_{M}\right\}$

where $\operatorname{End}(M)$ denotes the endomorphism semigroup of $M$ and $\operatorname{Aut}(M)$ denotes the automorphism group of $M$.

Proof. The implications (i) $\Rightarrow($ ii) $\Rightarrow($ iii) $\Rightarrow$ (iv) are trivial. To show (iv) $\Rightarrow$ (i), we prove the contraposition. Suppose $\chi_{M}$ is not a monomorphism. Then there exist $N \in \mathscr{M}$ and $f, g \in[N \rightarrow M]$ such that $f \neq g$ and $\chi_{M^{\circ}} f=\chi_{M^{\circ}} g$. Take $x \in N$ such that $f(x) \neq g(x)$. We put $u=f(x), v=g(x)$. We define $h: M \rightarrow M$ by:

$$
h(z)= \begin{cases}v & \text { if } \quad z=u \\ u & \text { if } z=v \\ z & \text { otherwise }\end{cases}
$$

It is easy to see that $h \in \operatorname{Aut}(M)$, so that $|\operatorname{Aut}(M)|>1$.

A model $M \in \mathscr{M}$ is said to be reduced if $\chi_{M}$ is a monomorphism. 


\subsection{Structure of $\mathscr{K}_{i}(\Omega)$}

Theorem 4.8. Let $M=\langle W ; r, v\rangle$ be any model in $\mathscr{M}$, and suppose $(x, y) \in r(S, t)$. Then $\left(\chi_{M}(x), \chi_{M}(y)\right) \in R(S, t)$.

Proof. $(i=3)$ : Let $\chi_{M}(x)=\Gamma \rightarrow \Delta$ and $\chi_{M}(y)=\Gamma^{\prime} \rightarrow \Delta^{\prime}$. Suppose, by way of contradiction, that $\left(\chi_{M}(x), \chi_{M}(y)\right) \notin R(S, t)$. Then, by thedefini tion of $R$, for some $u \leq t$, we have $\Gamma_{S u} \ddagger \Gamma^{\prime}$ or $\Gamma_{o u} \ddagger \Gamma_{o_{u}}^{\prime}$. Suppose $\Gamma_{S u} \notin \Gamma^{\prime}$. Then there exists an $\alpha$ such that $[S u] \alpha \in \Gamma$ and $\alpha \notin \Gamma^{\prime}$. Then by the Fundamental Theorem of Universal Model, we have $\chi_{M}(x) \vDash$ $[S u] \alpha$ and $\chi_{M}(y)=1 \alpha$. Hence, by the definition of $\chi_{M}$, we have $x \vDash$ $[S u] \alpha$ and $y=1 \alpha$. Since $(x, y) \in r(S, t) \subseteq r(S, u)$, this is a contradiction. Next, suppose $\Gamma_{o u} \ddagger \Gamma_{o u}^{\prime}$. Then, similarly as above, for some $\alpha$ we have $x \vDash[O u] \alpha$ and $y=[O u] \alpha$. Since $(x, y) \in r(O, u)$ and $r(O, u)$ is transitive, we have a contradiction.

The cases $(i=4)$ and $(i=5)$ may be treated likewise.

Let $M, N \in \mathscr{M}$. We write $M \equiv N(\bmod \Omega)$ if Image $\left(\chi_{M}\right)=\operatorname{Image}\left(\chi_{N}\right)$. (We should write $\chi_{M}^{\Omega}$ (or $\chi_{N}^{\Omega}$ ) in place of $\chi_{M}$ (or $\chi_{N}$ ) if we wish to emphasize the dependence of $\chi$ on $\Omega$.) We say $M$ is equivalent (modulo $\Omega$ ) to $N$ if $M \equiv N(\bmod \Omega)$. Among the models equivalent to $M$, we will be interested in finding the simplest one. Let $M=\langle W ; r, v>\in \mathscr{M}$. We define its relational closure $\bar{M}=\langle W ; \bar{r}, v\rangle$ by letting $\left(w, w^{\prime}\right) \in \bar{r}(S, t)$ iff $\left(\chi_{M}(w), \chi_{M}\left(w^{\prime}\right)\right) \in R(S, t)$. By the above theorem we see $r \subseteq \bar{r}$ (, i.e., $r(S, t) \subseteq \bar{r}(S, t)$ for any $S, t$.) We can prove by induction that $1_{W}: M \rightarrow \bar{M}$ is an isomorphism. Thus, $\bar{r}$ is the largest among the relations $r^{\prime}$ on $W$ such that $\left\langle W ; r^{\prime}, v\right\rangle$ is equivalent to $M$. We say $M \in \mathscr{M}$ is relationally closed if $M=\bar{M}$. Now, let $M=\langle W ; r, v\rangle$ be relationally closed. An equivalence $\sim$ on $W$ is called a congruence if $w \sim w^{\prime}$ implies $\chi_{M}(w)$ $=\chi_{M}\left(w^{\prime}\right)$. In this case, we can naturally define its quotient model $M / \sim$ $=\langle\tilde{W} ; \tilde{r}, \tilde{v}>$ by:

(1) $\widetilde{W}=W / \sim=\{[w] \mid w \in W\}$

(2) $\left([w],\left[w^{\prime}\right]\right) \in \tilde{r}(S, t)$ iff $\left(w, w^{\prime}\right) \in r(S, t)$

(3) Let $p \in \operatorname{Pr} \cup\{\perp\}$. If $p \in \Omega$ then $[w] \in \tilde{v}(p)$ iff $w \in v(p)$, otherwise 
$\tilde{v}(p)$ is arbitrary

where $[w]$ denotes the equivalence class containing $w$. It is easy to see that $M / \sim$ is well-defined (up to the arbitrariness of $\tilde{v}(p)$ for $p \notin \Omega$ ) and $M \equiv M / \sim$. (The canonical map [ ]:M $M / \sim$ is a $p$-morphism if $\Omega$ $=\mathrm{Wff}$, and it is a homomorphism in any case.)

Suppose $M, N$ are relationally closed, and let $f \in[M \rightarrow N]$ be an epimorphism. Then, $\sim \subseteq M \times M$ defined by $w \sim w^{\prime}$ iff $f(w)=f\left(w^{\prime}\right)$ is a congruence, and we see $M / \sim$ is isomorphic to $N$. We write this as $M / f$ $\simeq N$.

Let $M \in \mathscr{M}$. By definition of $\chi_{M}, \chi_{M}\left(=\chi_{\bar{M}}\right)$ induces the largest congruence among the congruences on $M$. Hence we have:

Theorem 4.9. For any $M \in \mathscr{M}$, there uniquely (up to isomorphism) exists a reduced $N \in \mathscr{M}$ such that $M \equiv N$. Namely, $N$ is given by $N \simeq \bar{M} / \chi_{M}$.

Schematically, we have the following diagram:

$$
M \stackrel{1 W}{\longrightarrow} \bar{M} \stackrel{\chi_{M}}{\longrightarrow} \bar{M} / \chi_{M} \stackrel{\substack{\text { inclusion } \\ \text { map }}}{\longrightarrow} U(\Omega)
$$

Our argument in this chapter has been relative to $\Omega$. We end this chapter by giving a definition which does not depend on $\Omega$. Let $M$ $=\langle W ; r, v\rangle$ and $M^{\prime}=\left\langle W^{\prime} ; r^{\prime}, v^{\prime}\right\rangle$ be two KTi-models. We say $M$ and $M^{\prime}$ are strongly isomorphic if there is a bijection $f: M \rightarrow M^{\prime}$ which preserves the model structure, i.e., $f$ is a bijection such that

(1) For any $x, y \in W,(f(x), f(y)) \in r^{\prime}(S, t)$ iff $(x, y) \in r(S, t)$.

(2) For any $p \in \operatorname{Pr} \cup\{\perp\}$ and $w \in W, w \in v(p)$ iff $r(w) \in v^{\prime}(p)$.

\section{Chapter 5}

\section{S5 Model Theory}

In this chapter we give a complete classification of S5 models under the equivalence $\equiv(\bmod W f f)$. First, we need some general discussions. 


\subsection{Lindenbaum Algebra of KTi}

Let us define a relation $\leq^{*} \subseteq \mathrm{Wff} \times \mathrm{Wff}$ by $\alpha \leq^{*} \beta$ iff $\vdash \alpha \rightarrow \beta$ (in GTi). (As usual, we discuss by fixing a logical system KTi.) Furthermore, define $\sim \subseteq \mathrm{Wff} \times \mathrm{Wff}$ by $\alpha \sim \beta$ iff $\alpha \leq^{*} \beta$ and $\beta \leq^{*} \alpha$. $\leq^{*}$ is reflexive since $\vdash \alpha \rightarrow \alpha$. $\leq^{*}$ is transitive since $\vdash \alpha \rightarrow \beta$ and $\vdash \beta \rightarrow \gamma$ implies $\vdash \alpha \rightarrow \beta$. Hence $\sim$ is an equivalence relation. We may regard Wff as an algebra $<$ Wff; $\wedge, \vee, \neg, \supset,\{[S t] \mid S \in S p, t \in T\}>$. By the following lemma, we see that $\sim$ is a congruence on the algebra Wff. (For the definition of algebra and congruence, we refer to Grätzer [7].)

Lemma 5.1. Suppose $\alpha \sim \alpha^{\prime}$ and $\beta \sim \beta^{\prime}$. Then,

(i) $\alpha \wedge \beta \sim \alpha^{\prime} \wedge \beta^{\prime}$

(ii) $\alpha \vee \beta \sim \alpha^{\prime} \vee \beta^{\prime}$

(iii) $\neg \alpha \sim \neg \alpha^{\prime}$

(iv) $\alpha \supset \beta \sim \alpha^{\prime} \supset \beta^{\prime}$

(v) $[S t] \alpha \sim[S t] \alpha^{\prime} \quad($ for any $S \in S p, t \in T)$

Proof. Left to the reader.

By this lemma, one can define the quotient algebra $\boldsymbol{B}=<B ; \wedge, \vee$, $\neg, \supset,\{[S t] \mid S \in S p, t \in T\}>$, where $B=\mathrm{Wff} / \sim$. We will call this algebra the Lindenbaum algebra of $\mathrm{KT} i$. Let $\llbracket \rrbracket: \mathrm{Wff} \rightarrow \boldsymbol{B}$ denote the canonical homomorphism. We put $1=\llbracket T \rrbracket$ and $0=\llbracket \perp \rrbracket$.

Theorem 5.2. $<B ; \wedge, \vee, \neg, 0,1>$ is a Boolean algebra.

Proof. Left to the reader.

Let $\leq_{B} \subseteq B \times B$ denote the partial ordering induced by the Boolean structure of $B$, i.e., $a \leq_{B} b$ if and only if $a=a \wedge b$. Then we can easily verify that for any $\alpha, \beta \in \mathrm{Wff}, \alpha \leq^{*} \beta$ if and only if $\llbracket \alpha \rrbracket \leq_{B} \llbracket \beta \rrbracket$.

We will use the term theory as a synonym for a subset of Wff. Let $\Gamma$ be any theory. We say $\Gamma$ is consistent (or inconsistent) if so is the sequent $\Gamma \rightarrow$. If $\Gamma=\bar{\Gamma}=\mathrm{DC}(\Gamma)$, we say $\Gamma$ is (deductively) closed. Let $\boldsymbol{C}$ denote the set of all closed theories, i.e., 


$$
\mathbb{C}=\{\Gamma \subseteq \mathrm{Wff} \mid \Gamma=\bar{\Gamma}\}
$$

$\mathbb{C}$ is the set of fixed points of the retract DC: $2^{\text {wff }} \rightarrow 2^{\text {wff }}, C$ is partially ordered by the set inclusionship relation $\subseteq$. We define a mapping $\phi:$ Wff $\rightarrow \mathbb{C}$ by $\phi(\alpha)=\overline{\{\alpha\}}$. We say $\Gamma$ is finitely axiomatizable if $\bar{\Gamma}=\phi(\alpha)$ for some $\alpha \in \mathrm{Wff}$.

Lemma 5.3. $\llbracket \alpha \rrbracket \leq_{B} \llbracket \beta \rrbracket$ if and only if $\phi(\alpha) \supseteq \phi(\beta)$.

Proof. Only if part: By the assumption we have $\alpha \leq^{*} \beta$. Hence $\vdash \alpha \rightarrow \beta$. Take any $\pi \in \phi(\beta)=\overline{\{\beta\}}$. Then $\vdash \beta \rightarrow \pi$. Hence $\vdash \alpha \rightarrow \pi$, so that $\alpha \vdash \pi$. This means $\pi \in \phi(\alpha)$.

If part: Suppose $\phi(\alpha) \supseteq \phi(\beta)$. Since $\beta \in \phi(\beta) \subseteq \phi(\alpha)$, we have $\alpha \vdash \beta$, i.e., $\vdash \alpha \rightarrow \beta$. Hence $\llbracket \alpha \llbracket \leq_{B} \llbracket \beta \rrbracket$.

From this lemma we see that there uniquely exists an anti-order preserving injection $\iota: \mathbb{B} \rightarrow \boldsymbol{C}$ such that the diagram below commutes:

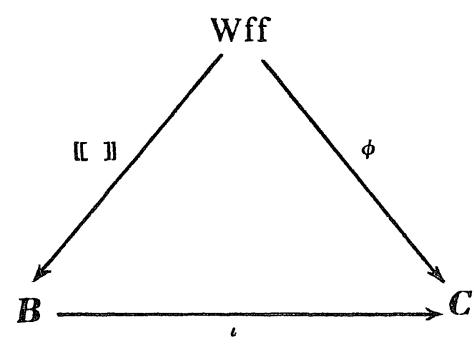

We note that $\iota$ is onto iff $\phi$ is onto. We give a sufficient condition for $\iota$ to be an anti-order isomorphism.

Lemma 5.4. If $\boldsymbol{B}$ satisfies the descending chain condition, then \& is an anti-order isomorphism.

Proof. Let $\Gamma$ be any element in $C$. Let $\alpha_{1}, \alpha_{2}, \ldots$ be an enumeration of $\Gamma$. Let $\beta_{n}=\alpha_{1} \wedge \cdots \wedge \alpha_{n}$. Let $\pi \in \phi\left(\beta_{n}\right)$. Then we have $\vdash \beta_{n} \rightarrow \pi$. Since $\vdash \Gamma \rightarrow \alpha_{i}(i=1,2, \ldots, n)$, we have $\vdash \Gamma \rightarrow \beta_{n}$. Hence $\vdash \Gamma \rightarrow \pi$. This means $\pi \in \bar{\Gamma}=\Gamma$. Therefore,

$$
\phi\left(\beta_{n}\right) \subseteq \Gamma
$$


Let $\pi \in \Gamma$. Then $\pi=\alpha_{n}$ for some $n$. Since $\vdash \beta_{n} \rightarrow \alpha_{n}$, we have $\pi=\alpha_{n}$ $\in \phi\left(\beta_{n}\right)$. Hence, together with (1), we have

$$
\Gamma=\bigcup_{n=1}^{\infty} \phi\left(\beta_{n}\right) .
$$

Since $\vdash \beta_{n+1} \rightarrow \beta_{n}$ for any $n$, we see $\llbracket \beta_{1} \rrbracket \geq_{B} \llbracket \beta_{2} \rrbracket \geq_{B} \cdots$. Since $\boldsymbol{B}$ satisfies descending chain condition, there exists an $m$ such that $\llbracket \beta_{m} \rrbracket \leq_{B} \llbracket \beta_{n} \rrbracket$ for any $n$. Then, by Lemma 5.3, we have $\phi\left(\beta_{m}\right) \supseteq \phi\left(\beta_{n}\right)$ for any $n$. Thus, by (1) and (2),

$$
\Gamma \supseteq \phi\left(\beta_{m}\right) \supseteq \bigcup_{n=1}^{\infty} \phi\left(\beta_{n}\right) \supseteq \Gamma
$$

This establishes the surjectivity of $\iota$. Thus we see that $\iota$ is an antiorder isomorphism.

\subsection{S5 Model Theory}

For any $n \geq 1$, we let the language $L_{n}=(\operatorname{Pr}(n), S p, T)$ be defined by:

(1) $\operatorname{Pr}(n)=\left\{p_{1}, p_{2}, \ldots, p_{n}\right\}$,

(2) $S p=\{O\}$,

(3) $T=\{1\}$.

Let us take any $L_{n}$ and fix it. In this section, we study KT5 over the language $L_{n}$, which is none other than the modal calculus S5 as we have seen in Fig. 1.1. Hence a KT5-model over $L_{n}$ will be called an S5model. Our aim is to determine the structure of the Universal Model $U=U(n)=U_{5}(\mathrm{Wff})$. We employ the more conventional notation $\square \alpha$ $(\diamond \alpha)$ in place of $[01] \alpha(<01>\alpha$, resp. $)$.

Let $\{ \pm\}^{n}$ denote the $n$-fold cartesian product of the doubleton set $\{+,-\}$. For any $\alpha \in \mathrm{Wff}$ and $\delta \in\{ \pm\}=\{+,-\}$, we put

$$
\alpha^{\delta}=\left\{\begin{array}{ccc}
\alpha & \text { if } & \delta=+ \\
\neg \alpha & \text { if } & \delta=-
\end{array}\right.
$$

We define a mapping

$$
\pi:\{ \pm\}^{n} \longrightarrow \text { Wff }
$$


by $\pi(\varepsilon)=p_{1}^{\varepsilon_{1}} \wedge \cdots \wedge p_{n}^{\varepsilon_{n}}$, where $\varepsilon=\varepsilon_{1} \cdots \varepsilon_{n}\left(\varepsilon_{i} \in\{ \pm\}\right)$. We put $\Pi=\operatorname{Image}(\pi)$. For any $E(\neq \emptyset) \subseteq\{ \pm\}^{n}$, we define an S5-model $M(E)=<W_{E} ; r_{E}, v_{E}>$ as follows:

(1) $W_{E}=E \times\{E\}$,

(2) $r_{E}(O, 1)=2^{W_{E} \times W_{E}}$,

(3) For any $(\varepsilon, E) \in W_{E},(\varepsilon, E) \in v\left(p_{i}\right)$ iff $\varepsilon_{i}=+$, where $\varepsilon=\varepsilon_{1} \cdots \varepsilon_{n}$, and $v(\perp)=\varnothing$.

Since $r_{E}(O, 1)$ is an equivalence relation, $M(E)$ is an 55 -model. We call this model the fragment model on $E$. We define its characteristic formula $\chi(E)$ by:

$$
\chi(E)=\wedge_{\varepsilon \in E} \diamond \pi(\varepsilon) \wedge \bigwedge_{\varepsilon \in\{ \pm\}^{n-E}} \neg \diamond \pi(\varepsilon) .^{8)}
$$

For any $(\varepsilon, E) \in M(E)$, we define its characteristic formula $\chi(\varepsilon, E)$ by:

$$
\chi(\varepsilon, E)=\pi(\varepsilon) \wedge \chi(E)
$$

Now, let $\left(M_{\lambda}\right)_{\lambda \in \Lambda}$ be an indexed family of S5-models, where $M_{\lambda}$ $\left.=<W_{\lambda} ; r_{\lambda}, v_{\lambda}\right\rangle$. We define their sum

$$
M=<W ; r, v>=\sum_{\lambda \in A} M_{\lambda}
$$

by:

(1) $W=\sum_{\lambda \in \Lambda} W_{\lambda}$ (disjoint union),

(2) $\left(w, w^{\prime}\right) \in r(O, 1)$ iff both $w$ and $w^{\prime}$ are in $W_{\lambda}$ for some $\lambda$ and $\left(w, w^{\prime}\right) \in r_{\lambda}(O, 1)$,

(3) $v(p)=\sum_{\lambda \in \Lambda} v_{\lambda}(p)$

An S5-model $M=\langle W ; r, v\rangle$ is said to be connected if $r(O, 1)$ $=2^{W \times W}$. It is easy to see that any S5-model $M$ may be expressed as a sum $\sum_{\lambda \in \Lambda} M_{\lambda}$ of their connected components $\left(M_{\lambda}\right)_{\lambda \in \Lambda}$.

Let $S$ be the sum of the family of all fragment models, i.e.,

$$
S=\sum_{\emptyset \neq E \subseteq\{ \pm\}^{n}} M(E) .
$$

8) For a finite set $A$ of wffs, we define $\underset{\alpha \in A}{\wedge} \alpha$ by $\alpha_{1} \wedge \cdots \wedge \alpha_{n}$, where $\alpha_{1}, \ldots, \alpha_{n}$ is any enumeration of $A$. 
We will show that $S$ is strongly isomorphic to $U$.

Lemma 5.5. Let an S5-model $M=\langle W ; r, v\rangle$ be connected and reduced (in the category $\mathscr{K}(\mathrm{Wff})$ ). Then $M$ is strongly isomorphic to some fragment model $M(E)$.

Proof. Let $E=\left\{\varepsilon \in\{ \pm\}^{n} \mid w \models \pi(\varepsilon)\right.$ (in $M$ ) for some $\left.w \in M\right\}$. Since for any $w \in W$ there uniquely exists an $\varepsilon \in E$ such that $w \models \pi(\varepsilon)$, we can define $\phi: W \rightarrow E$ by $\phi(w)=\varepsilon$. Suppose $\phi(w)=\phi\left(w^{\prime}\right)=\varepsilon$. We show by induction that for any $\alpha \in \mathrm{Wff}, w \vDash \alpha$ iff $w^{\prime} \vDash \alpha$. The case $\alpha \in \operatorname{Pr} \cup\{\perp\}$ is easily ascertained since $\phi(w)=\phi\left(w^{\prime}\right)$. The case $\alpha=\beta \supset \gamma$ is trivial by the definition of $\vDash$ and by induction hypothesis. Finally, we consider the case $\alpha=\square \beta$. Then, since $M$ is connected we see $w \models \square \beta$ iff $w^{\prime} \vDash \square \beta$. Hence, it follows that $\chi_{M}(w)=\chi_{M}\left(w^{\prime}\right)$. Since $M$ is reduced, we have $w=$ $w^{\prime}$, by Lemma 4.2. Thus we have proved that $\phi$ is a bijection. Since both $M$ and $M(E)$ are connected and $v_{E}(\phi(p))=v(p)$ for any $p \in \operatorname{Pr} \cup\{\perp\}$, we see that $M$ and $M(E)$ are strongly isomorphic.

Corollary 5.6. Let the assumptions be as in Lemma 5.5. Then the strong isomorphism $\phi: M \rightarrow M(E)$ is unique.

Proof. Since $M$ is reduced, we have $\operatorname{Aut}(M)=\left\{1_{M}\right\}$, by Lemma 4.7. Since a strong automorphism is an automorphism, we see that $\phi$ is unique.

Theorem 5.7. Let $M$ be connected and reduced. Suppose $w \vDash \chi(E)$ for some $w \in M$. Then $M$ is strongly isomorphic to $M(E)$.

Proof. By Lemma 5.5, we have only to prove: "If $E \neq E^{\prime}$ then $(\varepsilon, E)=\chi\left(E^{\prime}\right)$ for any $(\varepsilon, E) \in M(E)$." Suppose $E \neq E^{\prime}$ and $(\varepsilon, E) \vDash \chi\left(E^{\prime}\right)$ for some $(\varepsilon, E) \in M(E)$. Then we can take a $\delta$ such that $\delta \in E-E^{\prime}$ or $\delta \in E^{\prime}-E$. Suppose $\delta \in E-E^{\prime}$. Then $(\varepsilon, E) \vDash \diamond \pi(\delta)$. But, since $(\varepsilon, E)$ $\vDash \chi\left(E^{\prime}\right)$ and $\chi\left(E^{\prime}\right) \vdash \neg \diamond \pi(\delta)$, we have a contradiction. The case $\delta \in E^{\prime}-E$ may be treated similarly.

Now, let the Universal Model $U$ be expressed as the sum $\sum_{\lambda \in A} M_{\lambda}$ of its connected components. Then each $M_{\lambda}$ is reduced because $\chi_{U}=1_{U}$. 
By Lemma 5.5, $M_{\lambda}$ is strongly isomorphic to $M\left(E_{\lambda}\right)$ for a suitable $E_{\lambda}$. Let $\phi_{\lambda}: M_{\lambda} \rightarrow M\left(E_{\lambda}\right)$ be the unique strong isomorphism. Define $\phi: U$ $\rightarrow \sum_{\lambda \in \Lambda} M\left(E_{\lambda}\right)$ by $\phi(w)=\phi_{\lambda}(w)$ where $\lambda$ is the unique index such that $w \in M_{\lambda}$. Since $\phi$ is a strong isomorphism, we have the following commutative diagram:

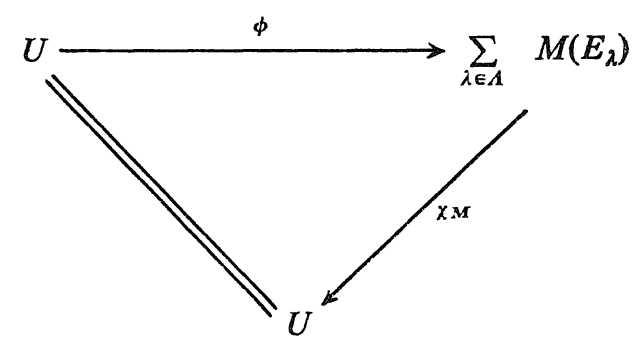

Hence, $\chi_{M}$ is also a strong isomorphism. Suppose $E_{\lambda}=E_{\mu}$ for some $\lambda$ $\neq \mu$. Then it is clear that $\operatorname{Aut}\left(\Sigma M\left(E_{\lambda}\right)\right) \supsetneqq\{1\}$. But, by Lemma 4.7, it is contrary to the fact that $\chi_{M}$ is a monomorphism. Thus we have:

$$
E_{\lambda} \neq E_{u} \quad \text { if } \quad \lambda \neq \mu \text {. }
$$

Now, take any $E(\neq \emptyset) \subseteq\{ \pm\}^{n}$. By Theorem 4.8, we see Image $\left(\chi_{M(E)}\right)$ is connected. Hence it is contained in some $M_{\lambda}$, i.e., $\operatorname{Image}\left(\chi_{M(E)}\right) \subseteq M_{\lambda}$. Take any $(\varepsilon, E) \in M(E)$. Then,

$$
(\varepsilon, E) \vDash \chi(E) \quad(\text { in } M(E)) .
$$

By the definition of $\chi_{M(E)}$,

$$
\chi_{M(E)}(\varepsilon, E) \vDash \chi(E) \quad(\text { in } U) .
$$

Hence,

$$
\chi_{M(E)}(\varepsilon, E) \vDash \chi(E) \quad\left(\text { in } M_{\lambda}\right)
$$

By applying $\phi$, we have

$$
\phi\left(\chi_{M(E)}(\varepsilon, E)\right) \vDash \chi(E) \quad\left(\text { in } M\left(E_{\lambda}\right)\right)
$$

Therefore by Theorem 5.7, we have $E=E_{\lambda}$. Thus we have proved the following

Theorem 5.8. $U$ is strongly isomorphic to $S$. 
Similarly, we have

Theorem 5.9. Let $M$ be reduced. Then $M$ is strongly isomorphic to $\sum_{E \in E} M(E)$ for some $E \subseteq 2^{\{ \pm\}^{n}}-\{\emptyset\}$.

Proof. Let $M=\sum_{\lambda \in \Lambda} M_{\lambda}$, where $M_{\lambda}(\lambda \in \Lambda)$ are reduced and connected. Since $M$ is reduced we have that $M_{\lambda}$ and $M_{\mu}$ are nonisomorphic if $\lambda \neq \mu$ by considering the automorphism group of $M$. Hence by Lemma 5.5 we have the desired result.

Corollary 5.10. An isomorphism $\phi: M \rightarrow N$ between reduced models $M$ and $N$ is an strong isomorphism.

On the other hand, it is clear that $\sum_{E \in E} M(E)$ is reduced for any $\boldsymbol{E} \subseteq 2^{\{ \pm\}^{n}}-\{\emptyset\}$. Hence we have

Corollary 5.11. There are $2^{2^{n-1}}$ nonisomorphic reduced S5-models.

Theorem 5.9 gives a complete classification of reduced models up to (strong) isomorphism. We will further proceed to define for any model $M$ its characteristic function $X(M)$.

Let $w=\Gamma \rightarrow \Delta \in U$. By the isomorphism $\phi: U \rightarrow S$ established in Theorem 5.9, we will identify $w$ with $\phi(w)$. Hence $w$ may be written as $w=\Gamma \rightarrow \Delta=(\varepsilon, E)$. We define a mapping

$$
X_{U}: U \longrightarrow \mathrm{Wff}
$$

by $X_{U}(w)=\chi(\varepsilon, E)$, where $w=(\varepsilon, E)$. Furthermore, for any model $M$, we define

$$
X_{M}: M \longrightarrow \mathrm{Wff}
$$

by $X_{M}(w)=X_{U}\left(\chi_{M}(w)\right)$, where $\chi_{M}$ is the characteristic function

$$
\chi_{M}: M \longrightarrow U
$$

Then the following theorem enables us to replace the semantical relation $\vDash$ by the syntactical one $\vdash$.

Theorem 5.12. Let $M$ be any S5-model. Then for any $w \in M$ 
and $\alpha \in \mathrm{Wff}$ we have:

$$
w \vDash \alpha(\text { in } M) \text { if and only if } X_{M}(w) \vdash \alpha .
$$

Proof. Since $w \vDash \alpha$ iff $\chi_{M}(w) \models \alpha$ (in $U$ ), and since $X_{M}=X_{U^{\circ}} \chi_{M}$, it suffices to prove the case $M=U$. So, let $w=\Gamma \rightarrow \Delta=(\varepsilon, E)$. We prove by induction on the construction of $\alpha$ that

(a) if $w \vDash \alpha$ then $X_{U}(w) \vdash \alpha$

and

(b) if $w=\mid \alpha$ then $X_{U}(w) \vdash \neg \alpha$.

$\alpha \in \operatorname{Pr} \cup\{\perp\}$ : The case $\alpha=\perp$ is trivial. So, suppose $\alpha=p_{i} \in \operatorname{Pr}$.

(a): Since $(\varepsilon, E) \vDash p_{i}$, we have $\varepsilon_{i}=+$. Hence $\pi(\varepsilon) \vdash p_{i}$, so that $X_{U}(w)$ $=\chi(\varepsilon, E)=\pi(\varepsilon) \wedge \chi(E) \vdash p_{i}(=\alpha)$. The proof of $(\mathrm{b})$ is similar.

$\alpha=\beta \supset \gamma$ :

(a): Since $w \vDash \beta \supset \gamma$, it follows that $w=\beta$ or $w \vDash \gamma$. Suppose $w=\beta$. Then by induction hypothesis, we have $X_{U}(w) \vdash \neg \beta$. Since $\neg \beta \vdash \beta \supset \gamma$, we have $X_{U}(w) \vdash \alpha$. The case $w \vDash \gamma$ may be treated similarly.

(b): Since $w \neq \beta \supset \gamma$, it follows that $w \vDash \beta$ and $w=\gamma$. By induction hypothesis, we have $X_{U}(w) \vdash \beta$ and $X_{U}(w) \vdash \neg \gamma$. Hence, $X_{U}(w) \vdash \beta \wedge \neg \gamma$. Since $\beta \wedge \neg \gamma \vdash \neg(\beta \supset \gamma)$, we have $X_{U}(w) \vdash \neg \alpha$.

$\alpha=\square \beta$ :

(a): Since $(\varepsilon, E) \vDash \square \beta$, we have for any $\delta \in E,(\delta, E) \vDash \beta$. By induction hypothesis, $\pi(\delta) \wedge \chi(E) \vdash \beta$ for any $\delta \in E$. Hence, we have:

$$
\vdash \underset{\delta \in E}{\vee} \pi(\delta), \chi(E) \rightarrow \beta
$$

Now, since $\vdash \rightarrow \underset{\delta \in\{ \pm\}^{n}}{\vee} \pi(\delta)$ and $\vdash \chi(E) \rightarrow \neg \pi(\delta)$ for any $\delta \notin E$, we have

$$
\vdash \chi(E) \rightarrow \underset{\delta \in E}{\vee} \pi(\delta)
$$

Hence, from (1) and (2) we obtain

$$
\vdash \chi(E) \rightarrow \beta
$$

From this, by $(\rightarrow \neg)$ and $(\rightarrow \square)$, we have $\chi(E) \vdash \square \beta$ as desired. 
(b) Since $(\varepsilon, E)=\square \beta$, we have, for some $\delta \in E,(\delta, E) \neq \beta$. By induction hypothesis, we have

$$
\vdash \pi(\delta), \chi(E) \rightarrow \neg \beta
$$

Let $\chi(E)=\diamond \pi\left(\varepsilon_{1}\right) \wedge \cdots \wedge \diamond \pi\left(\varepsilon_{i}\right) \wedge \neg \diamond \pi\left(\varepsilon_{i+1}\right) \wedge \cdots \wedge \neg \diamond \pi\left(\varepsilon_{j}\right)$. Then from (4) we can construct the following proof figure, which proves (b).

\begin{tabular}{|c|c|}
\hline$\beta, \pi(\delta), \square \neg \pi\left(\varepsilon_{i+1}\right), \ldots, \square \neg \pi\left(\varepsilon_{j}\right) \rightarrow \square \neg \pi\left(\varepsilon_{1}\right), \ldots, \square \neg \pi\left(\varepsilon_{i}\right)$ & \multirow{5}{*}{$\begin{array}{l}(\square \rightarrow) \\
(\rightarrow \neg) \\
-\quad(\rightarrow \square) \\
-\quad \text { (extension) }\end{array}$} \\
\hline$\square \beta, \pi(\delta), \square \neg \pi\left(\varepsilon_{i+1}\right), \ldots, \square \neg \pi\left(\varepsilon_{j}\right) \rightarrow \square \neg \pi\left(\varepsilon_{1}\right), \ldots, \square \neg \pi\left(\varepsilon_{i}\right)$ & \\
\hline$\square \beta, \square \neg \pi\left(\varepsilon_{i+1}\right), \ldots, \square \neg \pi\left(\varepsilon_{j}\right) \rightarrow \square \neg \pi\left(\varepsilon_{1}\right), \ldots, \square \neg \pi\left(\varepsilon_{i}\right), \neg \pi(\delta)$ & \\
\hline$\square \beta, \square \neg \pi\left(\varepsilon_{i+1}\right), \ldots, \square \neg \pi\left(\varepsilon_{j}\right) \rightarrow \square \neg \pi\left(\varepsilon_{1}\right), \ldots, \square \neg \pi\left(\varepsilon_{i}\right), \square \neg \pi(\delta)$ & \\
\hline$\square \beta, \square \neg \pi\left(\varepsilon_{i+1}\right), \ldots, \square \neg \pi\left(\varepsilon_{j}\right) \rightarrow \square \neg \pi\left(\varepsilon_{1}\right), \ldots, \square \neg \pi\left(\varepsilon_{i}\right)$ & \\
\hline$\chi(E) \rightarrow \neg \square \beta$ & \\
\hline$\pi(\delta), \chi(E) \rightarrow \neg \square \beta$ & \\
\hline
\end{tabular}

In the above proof a double line ( $=$ ) means that several trivial applications of rules are omitted.

Now it is clear that (b) implies that if $w=\alpha$ then $X_{U}(w)<\alpha$. This completes the proof of the theorem.

Corollary 5.13. Let $\tilde{X}_{U}: U \rightarrow \boldsymbol{B}$ be defined by $\tilde{X}_{U}(w)=\llbracket X_{U}(w) \rrbracket$. Then $\tilde{X}_{U}$ is injective.

Proof. Take any $w=(\varepsilon, E)$ and $w^{\prime}=\left(\varepsilon^{\prime}, E^{\prime}\right)$ in $U$. Suppose $\tilde{X}_{U}(w)$ $=\tilde{X}_{U}\left(w^{\prime}\right)$. Then, by Theorem 5.12, $(\varepsilon, E) \vDash \pi\left(\varepsilon^{\prime}\right) \wedge \chi\left(E^{\prime}\right)$. Hence, clearly, $\varepsilon=\varepsilon^{\prime}$. By Theorem 5.7, we have $E=E^{\prime}$. Therefore $w=w^{\prime}$, which means $\tilde{X}_{U}$ is injective.

In the above proof we have also proved

Corollary 5.14. Let $w, w^{\prime} \in U$. Then

(1) $w \vDash X_{U}\left(w^{\prime}\right)$ if and only if $w=w^{\prime}$.

(2) $X_{U}(w) \vdash X_{U}\left(w^{\prime}\right)$ if and only if $w=w^{\prime}$ 
We extend $X_{U}: U \rightarrow$ Wff to

$$
X_{U}: 2^{U} \longrightarrow \mathrm{Wff}
$$

as follows. Let $P \subseteq W_{U}$. Then $X_{U}(P)$ is defined by:

$$
X_{U}(P)=\underset{w \in P}{\vee} X_{U}(w) .
$$

We note that newly defined $X_{U}$ may be regarded as an extension of the old one by identifying $w$ with $\{w\}$. Now, for any $\alpha \in$ Wff we can define its normal form norm $(\alpha)$ by

$$
\operatorname{norm}(\alpha)=X_{U}\left(P_{\alpha}\right)
$$

where $P_{\alpha}=\{w \in U \mid w \vDash \alpha$ (in $\left.U)\right\}$.

Theorem 5.15. For any $\alpha \in \mathrm{Wff}$, $\operatorname{norm}(\alpha) \sim \alpha$.

Proof. Let $w \in P_{\alpha}$. Then by Theorem 5.12, $\vdash X_{U}(w) \rightarrow \alpha$. Hence we have $\vdash \underset{w \in P}{\vee} X_{U}(w) \rightarrow \alpha$, i.e., $\vdash \operatorname{norm}(\alpha) \rightarrow \alpha$. We prove $\vdash \alpha \rightarrow \operatorname{norm}(\alpha)$ by means of the Completeness Theorem. Consider any S5-model $M$ and $w \in M$ such that $w \vDash \alpha$ (in $M$ ). Let $w^{\prime}=\chi_{M}(w)$. Then $w^{\prime} \vDash \alpha$ (in $U$ ), i.e., $w^{\prime} \in P_{\alpha}$. Since $w^{\prime} \vDash X_{U}\left(w^{\prime}\right)$, we have $w^{\prime}=\chi_{M}(w) \vDash \operatorname{norm}(\alpha)$. Hence, by the definition of $\chi_{M}, w \models \operatorname{norm}(\alpha)$. By the Completeness Theorem, we have $\vdash \alpha \rightarrow \operatorname{norm}(\alpha)$. Thus, we have proved $\operatorname{norm}(\alpha) \sim \alpha$.

We are now ready to study the mapping

$$
h: 2^{U} \longrightarrow \mathbb{B}
$$

defined by $h(P)=\llbracket X_{U}(P) \rrbracket$. First, we define

$$
\square: 2^{U} \longrightarrow 2^{U}
$$

by $\square P=\left\{w \in U \mid\left(w, w^{\prime}\right) \in r(O, 1) \Rightarrow w^{\prime} \in P\right\}$. Then $2^{U}$ may be considered as an algebra $2^{U}=\left\langle 2^{W_{U}} ; \cap, U, \square>\right.$. Furthermore, we consider $\boldsymbol{B}$ as an algebra $\boldsymbol{B}=<B ; \wedge, \vee, \square>$.

Theorem 5.16. $h: 2^{U} \rightarrow \boldsymbol{B}$ is an isomorphism.

Proof. Take any $\llbracket \alpha \rrbracket \in B$ and let $P_{\alpha}=\{w \in U \mid w \models \alpha\}$. Then by Theo- 
rem 5.15, we have $h\left(P_{\alpha}\right)=\llbracket \operatorname{norm}(\alpha) \rrbracket=\llbracket \alpha \rrbracket$. Hence $h$ is surjective. Next, take any $P, Q \subseteq U$ and suppose $P \neq Q$. We can take $w$ such that $w \in$ $P-Q$ or $w \in Q-P$. Suppose $w \in P-Q$. Then clearly,

$$
X_{U}(w) \vdash X_{U}(P)
$$

Suppose $X_{U}(w) \vdash X_{U}(Q)$. Then by Theorem 5.12, we have $w \models X_{U}(Q)$. Hence for some $w^{\prime} \in Q$ we have $w \vDash X_{U}\left(w^{\prime}\right)$. Then by Corollary 5.14, we see $w=w^{\prime}$. This is a contradiction since $w \notin Q$ and $w^{\prime} \in Q$. Thus, we see

$$
X_{U}(w) \propto X_{U}(Q)
$$

By (1) and (2), we have $X_{U}(P) \approx X_{U}(Q)$, i.e.,

$$
\llbracket X_{U}(P) \rrbracket \neq \llbracket X_{U}(Q) \rrbracket
$$

Thus, we see $h$ is injective.

Now, let $P, Q \in 2^{U}$.

(i) Since $X_{U}(P \cap Q) \vdash X_{U}(P)$ and $X_{U}(P \cap Q) \vdash X_{U}(Q)$, we have

$$
\vdash X_{U}(P \cap Q) \rightarrow X_{U}(P) \wedge X_{U}(Q)
$$

On the other hand, suppose $w \vDash X_{U}(P) \wedge X_{U}(Q)$, where $w \in U$. Then, by a method similar as above, we can prove $w \in P \cap Q$. Hence $w \vDash X_{U}(P \cap Q)$. Thus we see

$$
\vdash X_{U}(P) \wedge X_{U}(Q) \rightarrow X_{U}(P \cap Q)
$$

By (3) and (4), we have $h(P \cap Q)=h(P) \wedge h(Q)$.

(ii) That $h(P \cup Q)=h(P) \vee h(Q)$ is proved similarly.

(iii) First, take any $w \in U$ such that $w \models X_{U}(\square P)$. Then $w \in \square P$, so that for any $\left(w, w^{\prime}\right) \in r(O, 1)$ we have $w^{\prime} \in P$. Hence $w^{\prime} \vDash X_{U}(P)$. Thus, we have $w \vDash \square X_{U}(P)$. Therefore, we have

$$
\vdash X_{U}(\square P) \rightarrow \square X_{U}(P)
$$

Next, take any $w \in U$ such that $w \vDash \square X_{U}(P)$. Let $w^{\prime}$ be such that $\left(w, w^{\prime}\right)$ $\in r(O, 1)$. Then we have $w^{\prime} \vDash X_{U}(P)$. Hence $w^{\prime} \in P$. Then by the definition of $\square P$, we have $w \in \square P$. Hence $w \vDash X_{U}(\square P)$. Thus, we have 


$$
\vdash \square X_{U}(P) \rightarrow X_{U}(\square P)
$$

By (5) and (6), we have $h(\square P)=\square h(P)$.

Theorems 5.8 and 5.16 determines the structure of the Lindenbaum algebra of S5. Since the cardinality of $U(=S)$ is easily calculated as

$$
|U|=\sum_{i=1}^{2^{n}} i \cdot\left(\begin{array}{c}
2^{n} \\
i
\end{array}\right)=2^{n} \cdot 2^{2^{n}-1}
$$

the cardinality of $\boldsymbol{B}$ is given by

$$
|B|=2^{|U|}=2^{2^{n} \cdot 2^{2 n-1}} .
$$

As an example, we illustrate the structure of $U$ for $n=2$.
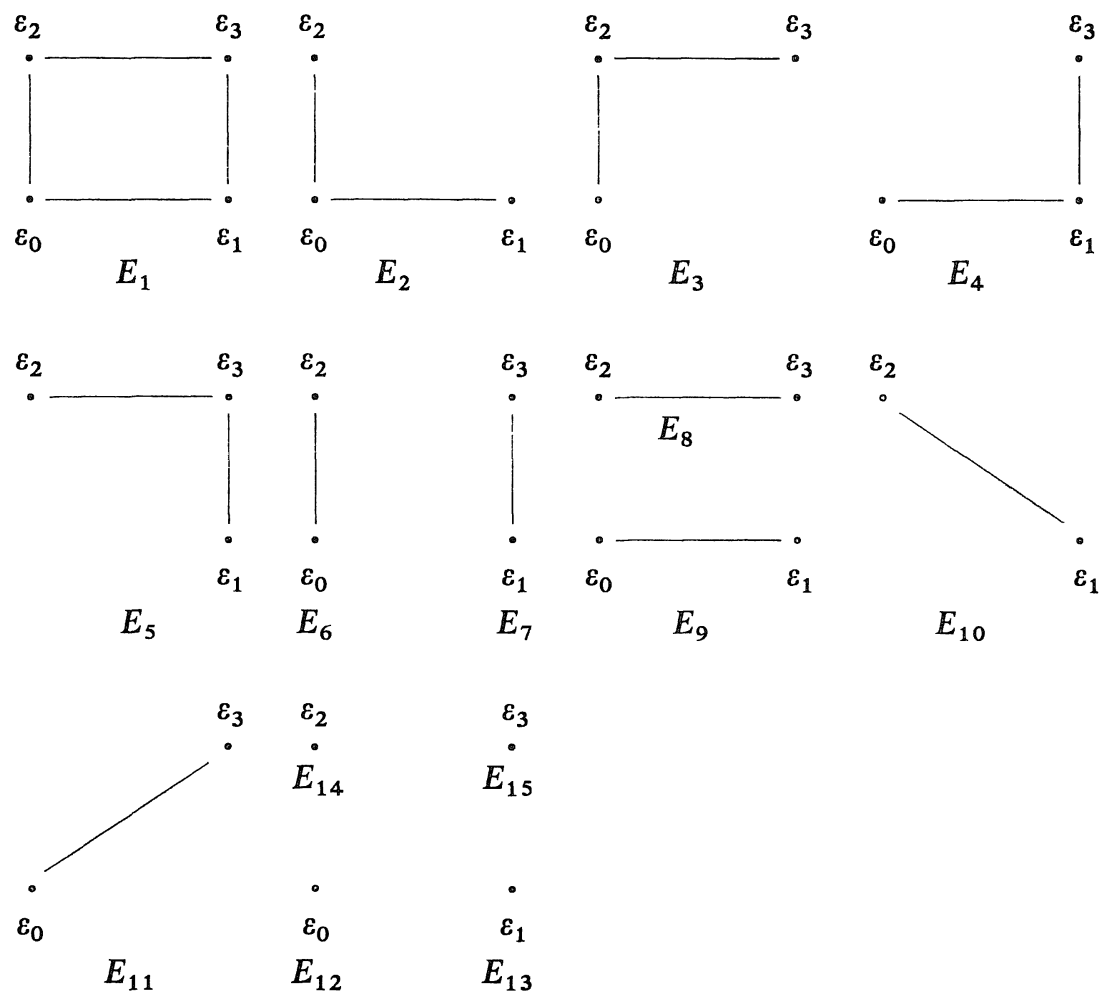

Fig. 5.1. Graphic representation of $U(2)^{9)}$

9) Define a relation $R_{0}$ by that $\left(\varepsilon_{i}, E_{k}\right) R_{0}\left(\varepsilon_{j}, E_{k}\right)$ iff the two points $\left(\varepsilon_{i}, E_{k}\right)$ and $\left(\varepsilon_{j}, E_{k}\right)$ are connected by a line in this figure. Then the reflexive and transitive closure of this relation gives the accessible relation of $U$. 
In the above figure, we have put $\varepsilon_{0}=\neg p_{1} \wedge \neg p_{2}, \varepsilon_{1}=p_{1} \wedge \neg p_{2}, \varepsilon_{2}=\neg p_{1}$ $\wedge p_{2}$ and $\varepsilon_{3}=p_{1} \wedge p_{2}$.

Finally, since $\boldsymbol{B}$ is finite, from Lemma 5.4, we have

Theorem 5.17. $\iota: B \rightarrow \mathbb{C}$ is an anti-order isomorphism.

Corollary 5.18. Every theory of S5 (over the language $L_{n}$ ) is finitely axiomatizable.

\section{Chapter 6}

\section{Applications}

In this chapter we study two puzzles, namely, the puzzle of three wise men and the puzzle of unfaithful wives, by applying the results we have obtained in the preceding chapters.

\subsection{The Wise Men Puzzle}

In this section, as an application of the Completeness Theorem, we give a model theoretic solution to the well-known puzzle of three wise men. We will work on the language $L=(P r, S p, T)$, where

$$
\begin{aligned}
& \operatorname{Pr}=\left\{p_{1}, p_{2}, p_{3}\right\}, \\
& S p=\left\{O, S_{1}, S_{2}, S_{3}\right\}, \\
& T=\{1\} .
\end{aligned}
$$

Since $T$ is a singleton set we will write, for example, $[S] \alpha$ in place of $[S 1] \alpha$. Now, the puzzle has been modified as follows by McCarthy [21, 22] so that it may be modelled in his knowledge system:

Let $S_{i}(i=1,2,3)$ denote the 3 wise men, and let $p_{i}$ be the sentence asserting that $S_{i}$ has a white spot on his forehead. The following are given as assumptions.

(A1) $p_{1} \wedge p_{2} \wedge p_{3} \cdots$ All spots are white.

(A2) $[0]\left(p_{1} \vee p_{2} \vee p_{3}\right)$-.. They all know that there is at least one white 
spot.

(A3) $[O]\left(\left\{S_{1}\right\} p_{2} \wedge\left\{S_{1}\right\} p_{3} \wedge\left\{S_{2}\right\} p_{1} \wedge\left\{S_{2}\right\} p_{3} \wedge\left\{S_{3}\right\} p_{1} \wedge\left\{S_{3}\right\} p_{2}\right)$-.- They all know that each can see the spots of the others.

(A4) $\left[S_{3}\right]\left[S_{2}\right] \neg\left[S_{1}\right] p_{1}-S_{3}$ knows that $S_{2}$ knows that $S_{1}$ doesn't know the color of his spot.

(A5) $\left[S_{3}\right] \neg\left[S_{2}\right] p_{2} \cdots S_{3}$ knows that $S_{2}$ doesn't know the color of his spot.

The problem is to deduce $\left[S_{3}\right] p_{3}$ ( $S_{3}$ knows that he has a white spot) from these assumptions.

Let $\alpha=(\mathrm{A} 1) \wedge(\mathrm{A} 2) \wedge(\mathrm{A} 3) \wedge(\mathrm{A} 4) \wedge(\mathrm{A} 5)$ and $\pi=\alpha \supset\left[S_{3}\right] p_{3}$. We will show that $\vdash \pi$ (in $\mathrm{K} 3$ ) by means of the completeness of K3-models. Namely, we show that $\pi$ is valid in all K3-models. So, by way of contradiction, suppose that there is a counter-model $M=\langle W ; r, v\rangle$ for $\pi$ such that $M=\pi$. This means that there is a world $w_{0} \in W$ such that

$$
w_{0} \vDash \alpha
$$

and

$$
w_{0}=\left[S_{3}\right] p_{3}
$$

(2) tells the existence of a world $w_{1}$ such that

$$
w_{0} \stackrel{S_{3}}{\longrightarrow} w_{1}
$$

and

$$
w_{1} \exists p_{3} \text {. }
$$

Since $w_{0} \vDash(\mathrm{A} 4) \wedge(\mathrm{A} 5)$, we have, by (3),

$$
w_{1} \vDash\left[S_{2}\right] \neg\left[S_{1}\right] p_{1}
$$

and

$$
w_{1}=\left[S_{2}\right] p_{2} \text {. }
$$

From (3) we have, by the definition of $r$,

$$
w_{0} \stackrel{o}{\longrightarrow} w_{1}
$$


Hence we have from (1)

$$
w_{1} \vDash\left\{S_{2}\right\} p_{3},
$$

that is, $w_{1} \vDash\left[S_{2}\right] p_{3}$ or $w_{1} \vDash\left[S_{2}\right] \neg p_{3}$. This, together with (4), implies

$$
w_{1} \vDash\left[S_{2}\right] \neg p_{3} .
$$

By (6) we see that there is a world $w_{2}$ such that

$$
w_{1} \stackrel{S_{2}}{\longrightarrow} w_{2}
$$

and

$$
w_{2}=p_{2} \text {. }
$$

From (5), (9) and (10) we have

$$
w_{2}=\left[S_{1}\right] p_{1}
$$

and

$$
w_{2}=p_{3} \text {. }
$$

By (10), since $r\left(S_{2}, 1\right) \subseteq r(O, 1)$, we have

$$
w_{1} \stackrel{o}{\longrightarrow} w_{2} \text {. }
$$

From (7) and (14), using the transitivity of $r(O, 1)$, we have

$$
w_{0} \stackrel{o}{\longrightarrow} w_{2} \text {. }
$$

Since $w_{0} \vDash(\mathrm{A} 3)$, we have

$$
w_{2} \vDash\left\{S_{1}\right\} p_{2} \wedge\left\{S_{1}\right\} p_{3} .
$$

From (11), (13) and (16) we have

$$
w_{2} \vDash\left[S_{1}\right] \neg p_{2}
$$

and

$$
w_{2} \vDash\left[S_{1}\right] \neg p_{3} \text {. }
$$

Now, (12) implies the existence of $w_{3} \in W$ such that 


$$
w_{2} \stackrel{s_{1}}{\longrightarrow} w_{3}
$$

and

$$
w_{3}=p_{1} \text {. }
$$

From (17), (18) and (19) we have

$$
w_{3}=1 p_{2}
$$

and

$$
w_{3}=p_{3} \text {. }
$$

We have

$$
w_{0} \stackrel{o}{\longrightarrow} w_{3}
$$

from (15) and (19). Then, since $w_{0} \vDash(\mathrm{A} 2)$, we have

$$
w_{3} \vDash p_{1} \vee p_{2} \vee p_{3}
$$

But, this is contradictory to (20)-(22). Thus, we have proved that $\pi$ is valid.

Note that we did not use the assumptions (A1) and $[O]\left(\left\{S_{2}\right\} p_{1}\right.$ $\left.\wedge\left\{S_{3}\right\} p_{1} \wedge\left\{S_{3}\right\} p_{2}\right)$. We illustrate the above inference in the following figure.

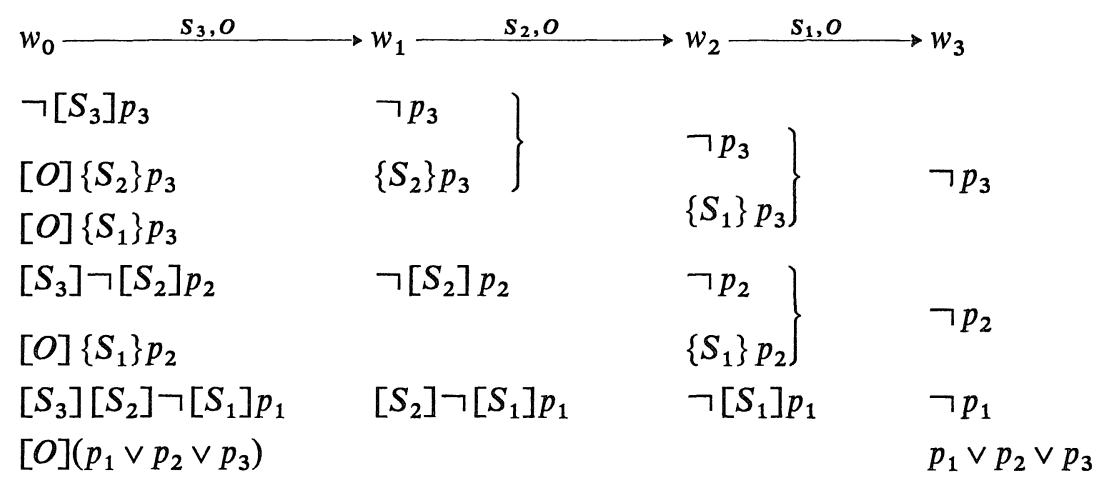

Fig. 6.1. Proof of the validity of $\pi$

For the sake of comparison, we give a formal proof of $\pi$ in GT3. It may be observed that these two proofs are essentially along the same 


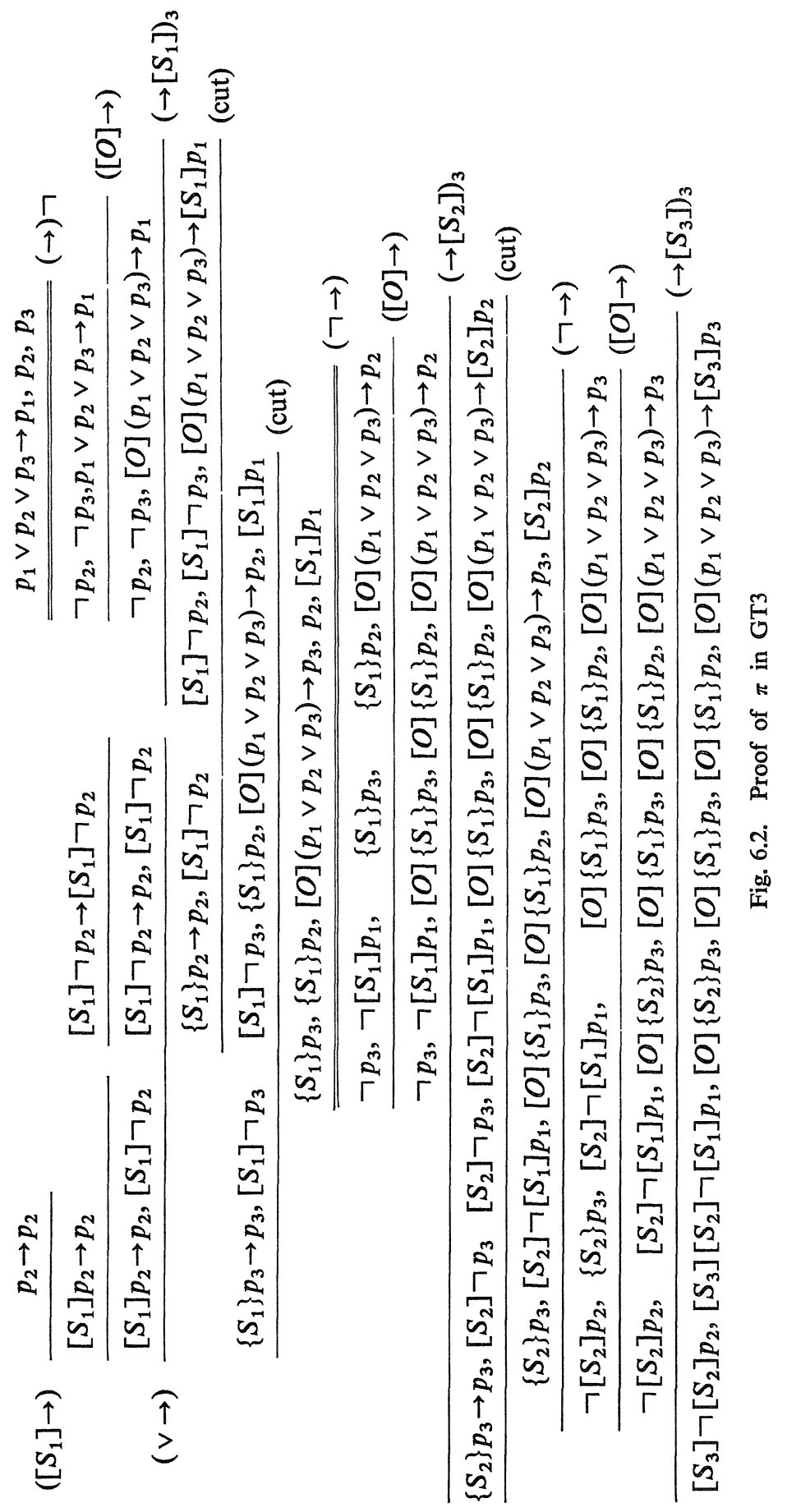


line.

\subsection{The Puzzle of Unfaithful Wives}

We begin by explaining the notions of knowledge base and knowledge set, which are fundamental for our formalization of the puzzle of unfaithful wives.

\subsubsection{Knowledge Set and Knowledge Base}

Let $L$ be any language. We consider in KT4 and KT5 over $L$. We will make the notion of the totality of one's knowledge explicit by the following definitions.

Definition 6.1. $K \subseteq \mathrm{Wff}$ is a knowledge set for $S t$ if $K$ satisfies the following conditions:

(KS1) $K$ is consistent.

(KS2) $K=[S t] \bar{K}$.

(KS3) If $K \vdash[S t] \alpha_{1} \vee \cdots \vee[S t] \alpha_{n}$ then $K \vdash \alpha_{i}$ for some $i(1 \leq i \leq n)$.

Definition 6.2. $B \subseteq \mathrm{Wff}$ is a knowledge base for $S t$ if $B$ satisfies the following conditions:

(KB1) $B$ is consistent.

(KB2) $B \subseteq[S t] \bar{B}$.

(KB3) If $B \vdash[S t] \alpha_{1} \vee \cdots \vee[S t] \alpha_{n}$ then $B \vdash \alpha_{i}$ for some $i(1 \leq i \leq n)$.

By (KS2) (or (KB2)) we see that any element in $K$ (or $B$, resp.) has the form $[S t] \alpha$. It is easy to see that if $B$ is a knowledge base for $S t$ then $[S t] \bar{B}$ is a knowledge set for St. We also note that the above definitions are relative to the logics KT4 and KT5.

Let $\Gamma \subseteq \mathrm{Wff}$ be consistent. We compare the following three conditions.

(1) If $\Gamma \vDash \alpha$ then $\Gamma \vdash \neg[S t] \alpha$.

(2) If $\Gamma \vdash[S t] \alpha_{1} \vee \cdots \vee[S t] \alpha_{n}$ then $\Gamma \vdash \alpha_{i}$ for some $i(1 \leq i \leq n)$.

(3) If $\Gamma \vdash\{$ St $\} \alpha$ then $\Gamma \vdash \alpha$ or $\Gamma \vdash \neg \alpha$. 
First, we consider in KT4.

Lemma 6.3. In KT4, we have (1) $\Rightarrow(2) \Rightarrow(3)$ but $(2) \nRightarrow(1)$.

Proof. (1) $\Rightarrow(2)$ : Suppose $\Gamma \vdash[S t] \alpha_{1} \vee \cdots \vee[S t] \alpha_{n}$ and $\Gamma \wedge \alpha_{i}$ for any $i$. Then by (1), we have $\Gamma \vdash \neg[S t] \alpha_{i}$ for any $i$. Then we can prove $\Gamma \vdash \perp$, which is contradictory to the consistency of $\Gamma$. (2) $\Rightarrow(3)$ : Trivial.

$(2) \not(1)$ : Since the disjunction property holds in KT4 (Theorem 3.12), the empty set $\emptyset$ is a knowledge base for any St. Let $\Gamma=\emptyset$. Then $\Gamma$ satisfies (2). Let $p \in P r .{ }^{10}$ ) Then neither $p$ nor $\neg[S t] p$ is provable in KT4. Hence, $\Gamma$ does not satisfy (1).

In KT5, we have the following

Lemma 6.4. In $\mathrm{KT} 5$, (1), (2), and (3) are equivalent.

Proof. $(1) \Rightarrow(2) \Rightarrow(3)$ are proved similarly as in Lemma 6.3.

$(3) \Rightarrow(1)$ : We prove the contraposition of (1) assuming (3). Suppose $\Gamma \times \neg[S t] \alpha$. Since $\vdash[S t][S t] \alpha \vee[S t] \neg[S t] \alpha$ in KT5, we have from (3), $\Gamma \vdash[S t] \alpha$. Hence $\Gamma \vdash \alpha$.

Note that $\emptyset$ is not a knowledge base in KT5. We now study the semantical characterization of knowledge sets. Let $M=\langle W ; r, v\rangle$ be any model (adequate for the logical system we have in mind). For any $w \in W$ and $(S, t) \in S p \times T$, we define $K_{w}(S t) \subseteq$ Wff by:

$$
K_{w}(S t)=\{[S t] \alpha \mid w \vDash[S t] \alpha\}
$$

Since, as we will see below, $K_{w}(S t)$ is a knowledge set for $S t$, we call it the knowledge set for St at $w$.

Lemma 6.5. $K_{w}(S t)$ is a knowledge set for $S t$.

Proof. We only prove (KS2). Let $[S t] \alpha \in K_{w}(S t)=K$. Then, we have $K \vdash \alpha$, i.e., $\alpha \in \bar{K}$. Hence $[S t] \alpha \in[S t] \bar{K}$. Let $[S t] \alpha \in[S t] \bar{K}$. Then

10) We need to assume that $\operatorname{Pr}$ is non-empty. In fact, if $\operatorname{Pr}=\varnothing$, we have Lemma 6.4 in place of this lemma, since in this case KT4 is equivalent to KT5. 
$\alpha \in \bar{K}$, i.e., $K \vdash \alpha$. Since any element in $K$ is of the form $[S t] \beta$, and the logical system is KT4 or KT5, we have $K \vdash[S t] \alpha$. Since $w \vDash K$, we have $w \vDash[S t] \alpha$, so that $[S t] \alpha \in K$.

Let $K$ be a knowledge set for St. We say $w \in M$ characterizes $K$ if $K=K_{w}(S t)$.

Theorem 6.6. Any knowledge set is characterizable.

Proof. Let $K$ be a knowledge set. Let $\Delta=\mathrm{Wff}-K_{S t}$. We show that the sequent $K \rightarrow[S t] \Delta$ is consistent. Suppose otherwise, so that $\vdash K \rightarrow[S t] \Delta$. Then for some finite set $\left\{\alpha_{1}, \ldots, \alpha_{n}\right\} \subseteq \Delta$ we have, $\vdash K \rightarrow$ $[S t] \alpha_{1}, \ldots,[S t] \alpha_{n}$. Here, we have $n \geq 1$ since $K$ is consistent by (KS1). Hence, by (KS3), there exists an $i(1 \leq i \leq n)$ such that $\vdash K \rightarrow \alpha_{i}$. By (KS2), we have $[S t] \alpha_{i} \in K$. This is a contradiction. Thus, $K \rightarrow[S t] \Delta$ is consistent. So, by the Generalized Completeness Theorem, we can take a model $M=\langle W ; r, v\rangle$ such that $w=K \rightarrow[S t] \Delta$, for some $w \in W$. Then, clearly, we have $K=K_{w}(S t)$.

\subsubsection{Informal Presentation of the Puzzle}

The puzzle of unfaithful wives is usually stated like this:

There was a country in which one million married couples inhabited. Among these one million wives, 40 wives were unfaithful. The situation was that each husband knew whether other men's wives are unfaithful but he did not know whether his wife is unfaithful. One day (call it the $1^{\text {st }}$ day), the King of the country publicized the following decree:

(i) There is at least one unfaithful wife.

(ii) Each husband knows whether other men's wives are unfaithful or not.

(iii) Every night (from tonight) each man must do his deduction, based on his knowledge so far, and try to prove whether his wife is unfaithful or not.

(iv) Each man, who has succeeded in proving that his wife is unfaithful, must chop off his wife's head next morning.

(v) Every morning each man must see whether somebody chops 
off his wife's head.

(vi) Each man's knowledge before this decree is publicized consists only of the knowledge about other men's wive's unfaithfulness.

The problem is "what will happen under this situation?" The answer is that on the $41^{\text {st }}$ day 40 unfaithful wives will be chopped off their heads. We will treat this puzzle in a formal manner.

\subsubsection{Formal Ttreatment of the Puzzle}

We will treat this puzzle by assuming that there are $k(\geq 1)$ married couples in the country. Then the language $L=(\operatorname{Pr}, S p, T)$ adequate for this puzzle will be:

$$
\begin{aligned}
& \operatorname{Pr}=\left\{p_{1}, \ldots, p_{k}\right\}, \\
& S p=\left\{O, S_{1}, \ldots, S_{k}\right\}, \\
& T=N^{+},
\end{aligned}
$$

where $S_{i}$ denotes $i^{\text {th }}$ husband, $p_{i}$ means that $S_{i}$ 's wife is unfaithful and $t \in T$ denotes $t^{t h}$ day. We employ KT5 over $L$ as our logical system. (Our argument henceforth can be carried out similarly in KT4 except for one point, where an essential use of Lemma 6.4 is necessary. This fact seems to suggest us that the negative introspective character of KT5 is essential for the solution of the puzzle.)

As in $\$ 5.2$, we define

$$
\pi:\{ \pm\}^{k} \longrightarrow \text { Wff }
$$

by $\pi\left(\varepsilon_{1} \cdots \varepsilon_{k}\right)=\bigwedge_{i=1}^{k} p_{i}^{\varepsilon_{i}}$. We put $\Pi=\operatorname{Image}(\pi)$ and $\Pi_{0}=\Pi-\left\{\bigwedge_{i=1}^{k} \bar{p}_{i}\right\}$, where $\bar{p}_{i}=\neg p_{i}$. We also use $\pi$ to denote arbitrary element in $\Pi$. Now, let $\Gamma$ denote the decree publicized by the King on the $1^{\text {st }}$ day, and $B_{\pi}\left(S_{i} n\right)$ $(i=1, \ldots, k)$ denote a knowledge base for $S_{i} n$ under the circumstance $\pi=\pi\left(\varepsilon_{1} \cdots \varepsilon_{k}\right) \in \Pi_{0}$. Let us put

$$
\left\ulcorner B_{\pi}\left(S_{i} n\right) \vdash \alpha\right\urcorner= \begin{cases}\top & \text { if } B_{\pi}\left(S_{i} n\right) \vdash \alpha \\ \perp & \text { otherwise }\end{cases}
$$


and

$$
\left\ulcorner B_{\pi}\left(S_{i} n\right) \uparrow \alpha\right\urcorner= \begin{cases}\top & \text { if } B_{\pi}\left(S_{i} n\right) \ngtr \alpha \\ \perp & \text { otherwise, }\end{cases}
$$

where $\alpha \in$ Wff. Then, as a formalization of the puzzle, we postulate the following identities:

$$
\begin{aligned}
& B_{\pi}\left(S_{i} 1\right)=\left[S_{i} 1\right] \Gamma \cup\left\{\left[S_{i} 1\right] p_{j}^{\varepsilon_{j}} \mid j \neq i, j=1, \ldots, k\right\} \quad \cdots E q(\pi, i, 1) \\
& B_{\pi}\left(S_{i} n+1\right) \\
& =\left[S_{i} n+1\right] B_{\pi}\left(S_{i} n\right) \cup\left\{\left[S_{i} n+1\right]\left[S_{j} n\right] p_{j} \mid B_{\pi}\left(S_{j} n\right) \vdash p_{j}, j=1, \ldots, k\right\} \\
& \quad \cup\left\{\left[S_{i} n+1\right] \neg\left[S_{j} n\right] p_{j} \mid B_{\pi}\left(S_{j} n\right) \uparrow p_{j}, j=1, \ldots, k\right\} \quad \cdots E q(\pi, i, n+1) \\
& \Gamma=\left\{[O 1] \bigvee_{i=1}^{k} p_{i}\right\} \cup\left\{[O 1]\left\{S_{i} 1\right\} p_{j} \mid j \neq i, i=1, \ldots, k, j=1, \ldots, k\right\} \\
& \cup\left\{[O 1]\left(\pi \supset\left(\left\ulcorner B_{\pi}\left(S_{i} n\right) \vdash p_{i}\right\urcorner \supset[O n+1]\left[S_{i} n\right] p_{i}\right)\right) \mid \pi \in \Pi_{0},\right. \\
& \quad i=1, \ldots, k, n \in T\} \\
& \cup\left\{[O 1]\left(\pi \supset\left(\left\ulcorner B_{\pi}\left(S_{i} n\right) \wedge p_{i}\right\urcorner \supset[O n+1] \neg\left[S_{i} n\right] p_{i}\right)\right) \mid \pi \in \Pi_{0},\right. \\
& \quad i=1, \ldots, k, n \in T\} \\
& \cup\left\{[O 1]\left(\left\ulcorner B_{\pi}\left(S_{i} n\right) \vdash \alpha\right\urcorner \supset[O 1]\left(\pi \supset\left[S_{i} n\right] \alpha\right)\right) \mid \pi \in \Pi_{0},\right. \\
& \quad i=1, \ldots, k, \alpha \in \mathrm{Wff}\}
\end{aligned}
$$

The informal meanings of the above equations are as follows:

$E q(\pi, i, 1)$ : Knowledge base for $S_{i} 1$ under $\pi$ consists of the knowledge about what the King says on the $1^{\text {st }}$ day and the knowledge about whether other men's wives are unfaithful.

$E q(\pi, i, n+1):$ If $S_{j}$ could prove $p_{j}$ in the $n^{t h}$ night, then $S_{i}$ knows on the $n+1^{s t}$ morning that $\left[S_{j} n\right] p_{j}$, since $S_{i}$ sees that $S_{j}$ chops off his wife's head in the $n+1^{\text {st }}$ morning. If $S_{j}$ could not prove $p_{j}$ in the $n^{t h}$ night, then $S_{i}$ knows in the $n+1^{s t}$ morning that $\neg\left[S_{j} n\right] p_{j}$, since $S_{i}$ sees that $S_{j}$ does not chop off his wife's head in the $n+1^{\text {st }}$ morning. 
$E q(*)$ : The meaning of the $1^{\text {st }}$ line of $E q(*)$ should be clear. The $2^{\text {nd }}$ and $3^{\text {rd }}$ lines mean that FOOL will know every morning whether anybody could prove the unfaithfulness of his wife in the previous night. The last line is an indirect definition of $B_{\pi}\left(S_{i} n\right)$.

Since the meta-notions such as knowledge base and provability $(\vdash)$ cannot be expressed directly in our language, we were forced to interpret the King's order into $\Gamma$ in a somewhat indirect fashion.

Now, if we read $E q(*)$ as the definition of $\Gamma$, then we find that the definition is circular, since in order that $\Gamma$ may be definable by $(*)$ it is necessary that $B_{\pi}\left(S_{i} n\right)$ are already defined, whereas $B_{\pi}\left(S_{i} n\right)$ are defined in terms of $\Gamma$ in $E q s(\pi, i, n)$. So, we will treat these equations as a system $\$=\left\{E q(\pi, i, n) \mid \pi \in \Pi_{0}, i=1, \ldots, k, n \in T\right\} \cup\{E q(*)\}$ of equations with the unknowns $\left\{B_{\pi}\left(S_{i} n\right) \mid \pi \in \Pi_{0}, i=1, \ldots, k, n \in T\right\}$ and $\Gamma$. We will solve $\$$ under the following conditions:

(\#) For any $\pi \in \Pi_{0}, \Gamma \cup\{\pi\}$ is consistent.

(\#\#) For any $\pi \in \Pi_{0}$ and $S_{i} n, B_{\pi}\left(S_{i} n\right)$ is a knowledge base for $S_{i} n$.

We think these conditions are natural in view of the intended meanings of $\Gamma$ and $B_{\pi}\left(S_{i} n\right)$.

For the sake of notational convenience, we consider $E=\{ \pm\}^{k}$ as a $k$-fold direct product of the vector space $\mathrm{GF}(2)=\{+(=1),-(=0)\}$ with addition $\oplus$. Thus, $\left\{e_{i}=-\cdots-+-\cdots-\mid i=1, \ldots, k\right\}$ forms a basis of $E$. We define a norm on $E$ by $\|\varepsilon\|=\left|\left\{i \mid \varepsilon_{i}=+\right\}\right|$, where $\varepsilon=\varepsilon_{1} \cdots \varepsilon_{k}{ }^{11}$ ) For any $\varepsilon=\varepsilon_{1} \cdots \varepsilon_{k} \in E$ and $i=1, \ldots, k$, we put

$$
\begin{aligned}
& \varepsilon(+i)=\varepsilon_{1} \cdots \varepsilon_{i-1}+\varepsilon_{i+1} \cdots \varepsilon_{k}, \\
& \varepsilon(-i)=\varepsilon_{1} \cdots \varepsilon_{i-1}-\varepsilon_{i+1} \cdots \varepsilon_{k},
\end{aligned}
$$

and for any $\pi=\pi(\varepsilon) \in \Pi$, we put

$$
\begin{aligned}
& \pi(+i)=\pi(\varepsilon(+i)), \\
& \pi(-i)=\pi(\varepsilon(-i))
\end{aligned}
$$

We also put $E_{0}=E-\{0\}=E-\{-\cdots-\}$.

11) For any $\varepsilon \in E$, we will employ the convention of denoting the $i^{\text {th }}$ coordinate of $\varepsilon$ by $\varepsilon_{i}$. 
Now, let us suppose that $<<B_{\pi}\left(S_{i} n\right) \mid \pi \in \Pi_{0}, i=1, \ldots, k, n \in T>, \Gamma>$ is a solution of $\$$ under the conditions (\#) and (\#\#). Then the following lemma holds.

Lemma 6.7. Let $\pi=\pi(\varepsilon) \in \Pi$ and $n \in T$. Then we have:

(i) If $n \geq\|\varepsilon(+i)\|$ then

$$
B_{\pi(+i)}\left(S_{i} n\right) \vdash p_{i}
$$

and

$$
B_{\pi(-\imath)}\left(S_{i} n\right) \vdash \bar{p}_{i} \quad\left(\text { if } \quad \pi(-i) \in \Pi_{0}\right) .
$$

(ii) If $n<\|\varepsilon(+i)\|$ then

$$
B_{\pi(+i)}\left(S_{i} n\right)=B_{\pi(-i)}\left(S_{i} n\right)
$$

and hence

$$
B_{\pi(+i)}\left(S_{i} n\right) \ngtr p_{i}
$$

and

$$
B_{\pi(-i)}\left(S_{i} n\right) \uparrow \bar{p}_{i}
$$

Proof. We first show that $B_{\pi(+i)}\left(S_{i} n\right)=B_{\pi(-i)}\left(S_{i} n\right)$ implies $B_{\pi(+i)}\left(S_{i} n\right)$ $\uparrow p_{i}$ and $B_{\pi(-i)}\left(S_{i} n\right)-\overline{p_{i}}$. Suppose $B_{\pi(+i)}\left(S_{i} n\right) \vdash p_{i}$. Then $B_{\pi(-i)}\left(S_{i} n\right) \vdash p_{i}$. Hence $[O 1]\left(\pi(-i) \supset\left(\top \supset[O n+1]\left[S_{i} n\right] p_{i}\right) \in \Gamma\right.$. So,

$$
\Gamma \vdash \pi(-i) \supset p_{i}
$$

On the other hand,

$$
\pi(-i) \vdash \overline{p_{i}}
$$

From (1) and (2), we have

$$
\pi(-i), \Gamma \vdash \perp
$$

This is contradictory to the condition $(\sharp)$. Therefore we have $B_{\pi(+i)}\left(S_{i} n\right)$ $\uparrow p_{i} . \quad B_{\pi(-i)}\left(S_{i} n\right) \times \bar{p}_{i}$ is proved similarly.

We now prove the lemma by induction on $n$. 
$n=1$ :

Proof of (i). Suppose $\|\varepsilon(+i)\|=1$. Then, since

$$
\begin{aligned}
& \vdash \overline{p_{1}}, \ldots, \overline{p_{i-1}}, \overline{p_{i+1}}, \ldots, \overline{p_{k}}, \bigvee_{i=1}^{k} p_{i} \rightarrow p_{i} . \\
& B_{\pi(+i)}\left(S_{i} 1\right) \vdash\left[S_{i} 1\right] \overline{p_{j}} \quad(j \neq i)
\end{aligned}
$$

and

$$
B_{\pi(+i)}\left(S_{i} 1\right) \vdash \bigvee_{i=1}^{k} p_{i}
$$

we have $B_{\pi(+i)}\left(S_{i} 1\right) \vdash p_{i}$. The rest of (i) is vacuously true, since $\pi(-i)$ $\in \Pi_{0}$.

Proof of (ii). Suppose $\|\varepsilon(+i)\|>1$. Then, $B_{\pi(+i)}\left(S_{i} 1\right)=B_{\pi(-i)}\left(S_{i} 1\right)$ follows directly from $E q(\pi(+i), i, 1)$ and $E q(\pi(-i), i, 1)$.

$n>1$ :

Proof of (i). First we show $B_{\pi(+i)}\left(S_{i} n\right) \vdash p_{i}$ from the assumption that $n=\|\varepsilon(+i)\|$. Since $n>1$, we can take $j \neq i$ such that $\varepsilon_{j}=+$. Then $\pi(+i)$ $=\pi(+i)(+j)$ and $\|\varepsilon(+i)(+j)\|=n>n-1$. By induction hypothesis, we therefore get $B_{\pi(+i)}\left(S_{j} n-1\right) \uparrow p_{j}$. Hence,

$$
\left[S_{i} n\right] \neg\left[S_{j} n-1\right] p_{j} \in B_{\pi(+i)}\left(S_{j} n\right) .
$$

On the other hand, since $\pi(-i)=\pi(-i)(+j)$ and $\|\varepsilon(-i)(+j)\|=n-1$, we have by induction hypothesis, $B_{\pi(-i)}\left(S_{j} n-1\right) \vdash p_{j}$. Hence, by $E q(*)$

$$
[01]\left(\pi(-i) \supset\left(T \supset[O n]\left[S_{j} n-1\right] p_{j}\right)\right) \in \Gamma \text {. }
$$

From (4), (5) and $E q(\pi(+i), i, n)$, we have $B_{\pi(+i)}\left(S_{i} n\right) \vdash \neg \pi(-i)$. Since $B_{\pi(+i)}\left(S_{i} 1\right) \vdash \pi(+i) \vee \pi(-i)$ and $B_{\pi(+i)}\left(S_{i} n\right) \supseteq\left[S_{i} n\right] \cdots\left[S_{i} 2\right] B_{\pi(+i)}\left(S_{i} 1\right)$, we have $B_{\pi(+i)}\left(S_{i} n\right) \vdash \pi(+i) \vee \pi(-i)$. Hence we have $B_{\pi(+i)}\left(S_{i} n\right) \vdash \pi(+i)$. Therefore, $B_{\pi(+i)}\left(S_{i} n\right) \vdash p_{i}$.

We next show that $B_{\pi(-i)}\left(S_{i} n\right) \vdash \bar{p}_{i}$ from the assumption that $n=$ $\|\varepsilon(+i)\|$. We can take $j \neq i$ such that $\varepsilon_{j}=+$. Then $\|\varepsilon(-i)(+j)\|=n-1$. By induction hypothesis, $B_{\pi(-i)}\left(S_{j} n-1\right) \vdash p_{j}$. Hence,

$$
\left[S_{i} n\right]\left[S_{j} n-1\right] p_{j} \in B_{\pi(-i)}\left(S_{i} n\right) .
$$

Since $\|\varepsilon(+i)(+j)\|=n$, we have by induction hypothesis, $B_{\pi(+i)} \uparrow p_{j}$. 
Hence,

$$
[O 1]\left(\pi(+i) \supset\left(\top \supset[O n] \neg\left[S_{j} n-1\right] p_{j}\right)\right) \in \Gamma
$$

From (6) and (7), by an argument similar as above, we conclude that $B_{\pi(-i)}\left(S_{i} n\right) \vdash \overline{p_{i}}$.

The case $n>\|\varepsilon(+i)\|$ is now easy, since we have

$$
B_{\pi}\left(S_{i} m+1\right) \supseteq\left[S_{i} m+1\right] B_{\pi}\left(S_{i} m\right),
$$

for any $m$.

Proof of (ii). We next consider the case $n<\|\varepsilon(+i)\|$. By induction hypothesis, $B_{\pi(+i)}\left(S_{i} n-1\right)=B_{\pi(-i)}\left(S_{i} n-1\right)$. Since $\|\varepsilon(+i)(+j)\| \geq\|\varepsilon(-i)(+j)\|$ $>n-1$ for any $j$, we have by induction hypothesis,

$$
B_{\pi(+i)(+j)}\left(S_{j} n-1\right)=B_{\pi(+i)(-j)}\left(S_{j} n-1\right)
$$

and

$$
B_{\pi(-i)(+j)}\left(S_{j} n-1\right)=B_{\pi(-i)(-j)}\left(S_{j} n-1\right) .
$$

Hence $B_{\pi(+i)}\left(S_{j} n-1\right) \uparrow p_{j}$ and $B_{\pi(-i)}\left(S_{j} n-1\right) \uparrow p_{j}$. Thus, we have $B_{\pi(+i)}\left(S_{i} n\right)$ $=B_{\pi(-i)}\left(S_{i} n\right)$ by $E q(\pi(+i), i, n)$ and $E q(\pi(-i), i, n)$.

Summarizing this lemma, we have:

Corollary 6.8. $B_{\pi(\varepsilon)}\left(S_{i} n\right) \vdash p_{i}$ if and only if $\varepsilon_{i}=+$ and $n \geq\|\varepsilon\|$.

We next prove the following lemma.

Lemma 6.9. For any $\pi=\pi(\varepsilon) \in \Pi_{0},\{\pi\} \cup \Gamma$ is complete. I.e., for any $\alpha \in \mathrm{Wff}$, either

$$
\vdash \pi, \Gamma \rightarrow \alpha
$$

or

$$
\vdash \alpha, \pi, \Gamma \rightarrow
$$

Proof. By induction on the construction of $\alpha$. First we note that, by condition (\#), it is impossible that both $\pi, \Gamma \rightarrow \alpha$ and $\alpha, \pi, \Gamma \rightarrow$ are provable. 
$\alpha \in \operatorname{Pr} \cup\{\perp\}:$

If $\alpha=p_{i}$ then we have $\pi \vdash p_{i}^{\varepsilon_{i}}$. Hence, clearly, $\vdash \pi, \Gamma \rightarrow \alpha$ or $\vdash \alpha, \pi$, $\Gamma \rightarrow$. If $\alpha=\perp$ then we have $\vdash \perp, \pi, \Gamma \rightarrow$.

$\alpha=\beta \supset \gamma$ :

Suppose $\vdash \pi, \Gamma \rightarrow \gamma$. Then we have $\vdash \pi, \Gamma \rightarrow \alpha$ by the following proof figure:

$$
\begin{gathered}
\ddots \because \vdots \\
\frac{\pi, \Gamma \rightarrow \gamma}{\beta, \pi, \Gamma \rightarrow \gamma} \\
\hline \pi, \Gamma \rightarrow \beta \supset \gamma
\end{gathered}
$$

Suppose $\vdash \beta, \pi, \Gamma \rightarrow$. Then we have $\vdash \pi, \Gamma \rightarrow \alpha$, similarly.

By induction hypothesis, we see that the remaining case is $\vdash \pi, \Gamma \rightarrow \beta$ and $\vdash \gamma, \pi, \Gamma \rightarrow$. Then, we have $\vdash \beta \supset \gamma, \pi, \Gamma \rightarrow$ by $(\supset \rightarrow)$.

$\alpha=\left[S_{i} n\right] \beta$ :

Suppose $\vdash \beta, \pi, \Gamma \rightarrow$. Then we can construct the following proof:

$$
\begin{array}{r}
\because \vdots \vdots \\
\beta, \pi, \Gamma \rightarrow \\
\hline\left[S_{i} n\right] \beta, \pi, \Gamma \rightarrow
\end{array}
$$

Suppose $\vdash \pi, \Gamma \rightarrow \beta$.

(A) We first consider the case $n \geq\|\varepsilon(+i)\|$.

(A1) The case $\pi=\pi(+i)$ :

In this case, noting that $[O 1]\left(\pi(+i) \supset\left(T \supset[O n+1]\left[S_{i} n\right] p_{i}\right)\right) \in \Gamma$ by Lemma 6.7, we first construct the following proof figure.

$$
\begin{gathered}
\frac{\perp \rightarrow}{\rightarrow \mathrm{T}} \frac{\left[S_{i} n\right] p_{i} \rightarrow\left[S_{i} n\right] p_{i}}{[O n+1]\left[S_{i} n\right] p_{i} \rightarrow\left[S_{i} n\right] p_{i}} \\
\frac{\pi(+i) \rightarrow \pi(+i)}{\frac{\mathrm{T} \supset[O n+1]\left[S_{i} n\right] p_{i} \rightarrow\left[S_{i} n\right] p_{i}}{\left[O(+i) \supset\left(\mathrm{T} \supset[O n+1]\left[S_{i} n\right] p_{i}\right), \pi(+i) \rightarrow\left[S_{i} n\right] p_{i}\right.}} \\
\frac{[O 1]\left(\pi(+i) \supset\left(\mathrm{T} \supset[O n+1]\left[S_{i} n\right] p_{i}\right)\right), \pi(+i) \rightarrow\left[S_{i} n\right] p_{i}}{\pi(+i), \Gamma \rightarrow\left[S_{i} n\right] p_{i}}
\end{gathered}
$$


Let $j \neq i$. Then, since $[O 1]\left\{S_{i} 1\right\} p_{j} \in \Gamma$, we have the following proof figure.

(2)

$$
\begin{array}{r}
\frac{p_{j}^{\varepsilon_{j}} \rightarrow p_{j}^{\varepsilon_{j}}}{\pi(+i) \rightarrow p_{j}^{\varepsilon_{j}}} \\
\frac{p_{j}^{\varepsilon_{j}} \rightarrow p_{j}^{\varepsilon_{j}}}{\frac{\left[S_{i} 1\right] p_{j}^{\varepsilon_{j}} \rightarrow\left[S_{i} n\right] p_{j}^{\varepsilon_{j} j}, \pi(+i) \rightarrow}{\left[S_{i} 1\right] \neg p_{j}^{\varepsilon_{j}}, \pi(+i) \rightarrow}} \\
\frac{\pi(+i),\left[S_{i} 1\right] p_{j}^{\varepsilon_{j}} \vee\left[S_{i} 1\right] \neg p_{j}^{\varepsilon_{j}} \rightarrow\left[S_{i} n\right] p_{j}^{\varepsilon_{j}}}{\frac{\pi(+i),[O 1]\left\{S_{i} 1\right\} p_{j} \rightarrow\left[S_{i} n\right] p_{j}^{\varepsilon_{j}}}{\pi(+i), \Gamma \rightarrow\left[S_{i} n\right] p_{j}^{\varepsilon_{l}}}}
\end{array}
$$

From (1) and (2) we have

$$
\vdash \pi(+i), \Gamma \rightarrow\left[S_{i} n\right] \pi(+i)
$$

(A2) The case $\pi=\pi(-i)$ :

We treat the critical case of $n=\|\varepsilon(+i)\|$. Then we see $\|\varepsilon(-i)\|$ $=n-1 \geq 1$, since $\pi(-i)=\pi \in \Pi_{0}$. So, we can take $j \neq i$ such that $\varepsilon_{j}=+$. Then, since $\|\varepsilon(+i)(+j)\|=n$ and $\|\varepsilon(-i)(+j)\|=n-1$, we have

$$
[O 1]\left(\pi(+i) \supset\left(\mathrm{T} \supset[O n] \neg\left[S_{j} n-1\right] p_{j}\right) \in \Gamma\right.
$$

and

$$
[O 1]\left(\pi(-i) \supset\left(\top \supset[O n]\left[S_{j} n-1\right] p_{j}\right) \in \Gamma .\right.
$$

Hence we obtain the following proof figure.

$$
\begin{aligned}
& \because \vdots \bullet^{\circ} \quad \because \vdots \bullet^{\circ} \\
& \pi(-i), \Gamma \rightarrow\left[S_{i} n\right]\left[S_{j} n-1\right] p_{i} \quad \Gamma \rightarrow\left[S_{i} n\right]\left(\pi(+i) \supset \neg\left[S_{j} n-1\right] p_{j}\right) \quad \because \vdots \bullet^{\circ} \\
& \begin{array}{ll}
\pi(-i), \Gamma \rightarrow\left[S_{i} n\right] \neg \pi(+i) & \pi(-i), \Gamma \rightarrow\left[S_{i} n\right](\pi(+i) \vee \pi(-i)) \\
\pi(-i), \Gamma \rightarrow\left[S_{i} n\right] \pi(-i)
\end{array}
\end{aligned}
$$

From the above proof, for any $n \geq\|\varepsilon(+i)\|$, it follows that

$$
\vdash \pi(-i), \Gamma \rightarrow\left[S_{i} n\right] \pi(-i) \text {. }
$$

Since $\pi=\pi(+i)$ or $\pi=\pi(-i)$, we have from (3) and (4), 
Using (5), we obtain the desired proof figure:

$$
\begin{gathered}
\cos ^{(5)} \\
\frac{\pi, \Gamma \rightarrow\left[S_{i} n\right] \pi}{\pi, \Gamma \rightarrow \beta} \\
\frac{\left[S_{i} n\right] \pi, \Gamma \rightarrow \beta}{\left[S_{i} n\right] \pi, \Gamma \rightarrow\left[S_{i} n\right] \beta}
\end{gathered}
$$

(B) We next consider the case $n<\|\varepsilon(+i)\|$.

Let $\varepsilon^{\prime}=\varepsilon \oplus e_{i}$. Then, by induction hypothesis, we have the following two cases.

(B1) $\vdash \pi\left(\varepsilon^{\prime}\right), \Gamma \rightarrow \beta$ :

The following proof figure takes care of this case.

$$
\begin{aligned}
& \because \vdots 0^{\circ} \quad \because \vdots 0^{\circ} \\
& \frac{\pi(\varepsilon), \Gamma \rightarrow \beta \quad \pi\left(\varepsilon^{\prime}\right), \Gamma \rightarrow \beta}{\pi(\varepsilon) \vee \pi\left(\varepsilon^{\prime}\right), \Gamma \rightarrow \beta} \\
& \frac{\pi, \Gamma \rightarrow\left[S_{i} n\right]\left(\pi(\varepsilon) \vee \pi\left(\varepsilon^{\prime}\right)\right)}{\pi, \Gamma \rightarrow\left[S_{i} n\right] \beta}
\end{aligned}
$$

(B2) $\vdash \beta, \pi\left(\varepsilon^{\prime}\right), \Gamma \rightarrow$ :

We first show that

$$
\vdash \pi, \Gamma \rightarrow<S_{i} n>\pi\left(\varepsilon^{\prime}\right)
$$

Suppose $\pi=\pi(+i)$. Then, by Lemma 6.7, we have $B_{\pi}\left(S_{i} n\right) \uparrow p_{i}$. Since $B_{\pi}\left(S_{i} n\right)$ is a knowledge base by condition (\#\#), we have $B_{\pi}\left(S_{i} n\right) \vdash$ $\neg\left[S_{i} n\right] p_{i}$ by Lemma 6.4. (Note that we are considering in KT5. Here we remark that this is the only point where we use the assumption that our logical system is KT5.) Then by $E q(*)$, we see that

$$
[O 1]\left(T \supset[O 1]\left(\pi \supset\left[S_{i} n\right] \neg\left[S_{i} n\right] p_{i}\right)\right) \in \Gamma \text {. }
$$

Hence we have 


$$
\vdash \pi, \Gamma \rightarrow<S_{i} n>\neg p_{i}
$$

Now, for any $\sigma, \tau \in \mathrm{Wff}$ we have

$$
1<S_{i} n>\sigma,\left[S_{i} n\right] \tau \rightarrow<S_{i} n>(\sigma \wedge \tau)
$$

as can be seen from the following proof figure.

$$
\begin{gathered}
\frac{\sigma, \tau \rightarrow \sigma \wedge \tau}{\neg(\sigma \wedge \tau), \tau \rightarrow \neg \sigma} \\
\frac{\left[S_{i} n\right] \neg(\sigma \wedge \tau),\left[S_{i} n\right] \tau \rightarrow\left[S_{i} n\right] \neg \sigma}{<S_{i} n>\sigma,\left[S_{i} n\right] \tau \rightarrow<S_{i} n>(\sigma \wedge \tau)}
\end{gathered}
$$

Now we can obtain (6) from (2), (7) and (8) (where we put $\sigma=\neg p_{i}$ and $\left.\tau=\wedge_{j \neq i} p_{j}^{\varepsilon_{j}}\right)$. The case $\pi=\pi(-i)$ may be treated similarly.

We can then construct the following proof figure:

$$
\begin{gathered}
\ddots \vdots \bullet^{\circ} \\
\frac{\frac{\beta, \pi\left(\varepsilon^{\prime}\right), \Gamma \rightarrow}{\beta, \Gamma \rightarrow \neg \pi\left(\varepsilon^{\prime}\right)}}{(6)} \\
\frac{\frac{\left[S_{i} n\right] \beta, \Gamma \rightarrow \neg \pi\left(\varepsilon^{\prime}\right)}{\left[S_{i} n\right] \beta, \Gamma \rightarrow\left[S_{i} n\right] \neg \pi\left(\varepsilon^{\prime}\right)}}{<, \Gamma \rightarrow<S_{i} n>\pi\left(\varepsilon^{\prime}\right)} \\
{\left[S_{i} n\right] \beta, \pi, \Gamma \rightarrow}
\end{gathered}
$$

$\alpha=[O n] \beta$ :

If $\vdash \beta, \pi, \Gamma \rightarrow$, then we have $\vdash[O n] \beta, \pi, \Gamma \rightarrow$ by $([O n] \rightarrow)$. So, suppose $\vdash \pi, \Gamma \rightarrow \beta$. Then we have the following two cases (C) and (D).

(C) The case $n \geq \max \{\|\varepsilon(+i)\| \mid i=1, \ldots, k\}$.

As in (A2) it is sufficient to prove the critical case of $n=\max \{\|\varepsilon(+i)\| 1$ $i=1, \ldots, k\}$. Let us put $I(\varepsilon)=\left\{i \mid \varepsilon_{i}=+\right\}$.

(C1) The case $I(\varepsilon) \neq\{1,2, \ldots, k\}$ :

In this case, we have $n=\|\varepsilon\|+1$. Consider any $i$ such that $\varepsilon_{i}=+$. Then we have $\pi=\pi(+i)$, and since $n-1 \geq\|\varepsilon\|=\|\varepsilon(+i)\|$, we have $B_{\pi}\left(S_{i} n\right.$ $-1) \vdash p_{i}$ by Lemma 6.7. Hence we have

$$
[O 1]\left(\pi \supset\left(\top \supset[O n]\left[S_{i} n-1\right] p_{i}\right)\right) \in \Gamma .
$$


So, we have

$$
\vdash \pi, \Gamma \rightarrow[O n]\left[S_{i} n-1\right] p_{i} \quad\left(\text { if } \quad \varepsilon_{i}=+\right. \text { ) }
$$

and hence

$$
\vdash \pi, \Gamma \rightarrow[O n] p_{i} \quad\left(\text { if } \quad \varepsilon_{i}=+\right. \text { ). }
$$

Let $D=\left\{\delta \in\{ \pm\}^{k} \mid I(\varepsilon) \subseteq I(\delta)\right\}$. Then, by (10) we have

$$
\vdash \pi, \Gamma \rightarrow[O n] \underset{\delta \in D}{\vee} \pi(\delta) .
$$

Now, take any $\delta \in D-\{\varepsilon\}$. Then we have $\|\delta\|>\|\varepsilon\|=n-1$. Since $\pi(\varepsilon)$ $\in \Pi_{0}$, we can take an $i$ such that $\varepsilon_{i}=+$. Then we have $\delta=\delta(+i)$. Since $\|\delta\|>n-1$, we have $B_{\pi(\delta)}\left(S_{i} n-1\right) \uparrow p_{i}$, by Lemma 6.7. Hence, we have

$$
[O 1]\left(\pi(\delta) \supset\left(\top \supset[O n] \neg\left[S_{i} n-1\right] p_{i}\right)\right) \in \Gamma .
$$

From this, together with (9), we have the following proof figure.

$$
\frac{\pi, \Gamma \rightarrow[O n]\left[S_{i} n-1\right] p_{i} \quad \Gamma \rightarrow[O n]\left(\pi(\delta) \supset \neg\left[S_{i} n-1\right] p_{i}\right)}{\underline{\pi, \Gamma \rightarrow[O n]\left(\left[S_{i} n-1\right] p_{i} \wedge \pi(\delta) \supset \neg\left[S_{i} n-1\right] p_{i}\right)}}
$$

From (11) and (12), we have

$$
\vdash \pi, \Gamma \rightarrow[O n] \pi
$$

(C2) The case $I(\varepsilon)=\{1,2, \ldots, k\}$ :

In this case, we have $\varepsilon=+\cdots+$ and $n=\|\varepsilon\|(=k)$. Let $\delta \in E_{0}-\{\varepsilon\}$. We can find an $i$ such that $\delta_{i}=+$. Then we have $n-1 \geq\|\delta\|=\|\delta(+i)\|$. Hence, by Lemma 6.7, we have $B_{\pi(\delta)}\left(S_{i} n-1\right) \vdash p_{i}$. Hence, we have

$$
[O 1]\left(\pi(\delta) \supset\left(\top \supset[O n]\left[S_{i} n-1\right] p_{i}\right)\right) \in \Gamma \text {. }
$$

On the other hand, since $n-1<\|\varepsilon\|=\|\varepsilon(+i)\|$, applying Lemma 6.7, we get $B_{\pi}\left(S_{i} n-1\right) \times p_{i}$. So, we have 


$$
[O 1]\left(\pi \supset\left(\top \supset[O n] \neg\left[S_{i} n-1\right] p_{i}\right)\right) \in \Gamma \text {. }
$$

Hence, we have

$$
\vdash \pi, \Gamma \rightarrow[O n] \neg\left[S_{i} n-1\right] p_{i}
$$

From (14) and (15), similarly as in (12), we obtain

$$
\left.\vdash \pi, \Gamma \rightarrow[O n] \neg \pi(\delta) \quad \text { (if } \delta \in E_{0}-\{\varepsilon\}\right) .
$$

By (16), together with the fact that $\vdash \Gamma \rightarrow[O n] \underset{\delta \in E_{0}}{\vee} \pi(\delta)$, we have

$$
\vdash \pi, \Gamma \rightarrow[O n] \pi \text {. }
$$

Now, by the results of (C1) and (C2), we can construct the following proof figure:

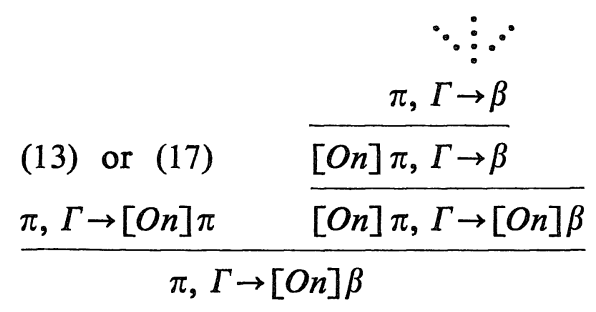

(D) The case $n<\max \{\|\varepsilon(+i)\| \mid i=1, \ldots, k\}$.

Let $D=\left\{\delta \in E_{0} \mid n<\max \{\|\delta(+i)\| \mid i=1, \ldots, k\}\right\}$. Take any $\delta \in E_{0}-D$ and choose an $i$ such that $\delta_{i}=+$. Then since $k>n$ by assumption, we have $n \geq \max \{\|\delta(+i)\| \mid i=1, \ldots, k\}>\|\delta\|=\|\delta(+i)\|$. Hence, we have

$$
B_{\pi(\delta)}\left(S_{i} n-1\right) \vdash p_{i}
$$

so that

$$
[O 1]\left(\pi(\delta) \supset\left(\top \supset[O n]\left[S_{i} n-1\right] p_{i}\right)\right) \in \Gamma
$$

On the other hand, we have

$$
B_{\pi}\left(S_{i} n-1\right)>p_{i}
$$

regardless of $\pi=\pi(+i)$ or $\pi=\pi(-i)$, so that

$$
[O 1]\left(\pi \supset\left(T \supset[O n] \neg\left[S_{i} n-1\right] p_{i}\right)\right) \in \Gamma .
$$


From (18) and (19), we have

$$
\vdash \pi, \Gamma \rightarrow[O n] \neg \pi(\delta) \quad \text { (if } \delta \in E_{0}-D \text { ). }
$$

From this, we have

$$
\vdash \pi, \Gamma \rightarrow[O n] \vee_{\delta \in D} \pi(\delta)
$$

Next, let $\delta \in D$. Then we can find $\gamma^{1}, \ldots, \gamma^{m} \in D$ such that $\gamma^{1}=\varepsilon$, $\gamma^{m}=\delta$ and $\left\|\gamma^{i} \oplus \gamma^{i+1}\right\|=1(i=1, \ldots, m-1)$. Now, take any $i$ such that $1 \leq i \leq m-1$. Let $\gamma^{i} \oplus \gamma^{i+1}=e_{j}$. Then we have $\gamma^{i}=\gamma^{i}(+j)$ or $\gamma^{i}=$ $\gamma^{i}(-j)$. Suppose, first, $\gamma^{i}=\gamma^{i}(+j)$. Then $\gamma^{i+1}=\gamma^{i} \oplus e_{j}=\gamma^{i}(-j)$. Since $\gamma^{i+1} \in D$, we have $n<\max \left\{\left\|\gamma^{i+1}(+l)\right\| \mid l=1, \ldots, k\right\}=\left\|\gamma^{i+1}(+j)\right\|$. Then we can apply (6) and obtain

$$
\vdash \pi\left(\gamma^{i}\right), \Gamma \rightarrow<S_{j} n>\pi\left(\gamma^{i+1}\right) .
$$

We can obtain (22) similarly for the case $\gamma^{i}=\gamma^{i}(-j)$. From (22), we get

$$
\vdash \pi\left(\gamma^{i}\right), \Gamma \rightarrow<O n>\pi\left(\gamma^{i+1}\right) .
$$

From (23) we obtain the following proof:

$$
\begin{aligned}
& \because \therefore \circ \\
& \frac{\pi\left(\gamma^{2}\right), \Gamma \rightarrow<O n>\pi\left(\gamma^{3}\right)}{[O n] \neg \pi\left(\gamma^{3}\right), \Gamma \rightarrow \neg \pi\left(\gamma^{2}\right)} \\
& \frac{\pi\left(\gamma^{1}\right), \Gamma \rightarrow<O n>\pi\left(\gamma^{2}\right) \quad \frac{[O n] \neg \pi\left(\gamma^{3}\right), \Gamma \rightarrow[O n] \neg \pi\left(\gamma^{2}\right)}{<O n>\pi\left(\gamma^{2}\right), \Gamma \rightarrow<O n>\pi\left(\gamma^{3}\right)}}{\pi\left(\gamma^{1}\right), \Gamma \rightarrow<O n>\pi\left(\gamma^{3}\right)} \\
& \frac{\pi\left(\gamma^{1}\right), \Gamma \rightarrow<O n>\pi\left(\gamma^{m-1}\right) \quad \frac{\pi\left(\gamma^{m-1}\right), \Gamma \rightarrow<O n>\pi\left(\gamma^{m}\right)}{<O n>\pi\left(\gamma^{m-1}\right), \Gamma \rightarrow<O n>\pi\left(\gamma^{m}\right)}}{\pi\left(\gamma^{1}\right), \Gamma \rightarrow<O n>\pi\left(\gamma^{m}\right)}
\end{aligned}
$$

Namely, we have 


$$
\vdash \pi, \Gamma \rightarrow<\text { On }>\pi(\delta) \quad \text { (if } \delta \in D)
$$

(Though the above proof applies only for $m>1$, (24) clearly holds even if $m=1$ (i.e., $\varepsilon=\delta$ ).)

Now, by induction hypothesis of the lemma, we have the following two cases.

(D1) $\vdash \pi(\delta), \Gamma \rightarrow \beta$ for any $\delta \in D$ :

Let $D$ be enumerated as $D=\left\{\delta^{1}, \ldots, \delta^{d}\right\}$. Then we have the following proof:

$$
\begin{array}{cc}
\ddots \because & \ddots \ominus^{\bullet} \\
\pi\left(\delta^{d-1}\right), \Gamma \rightarrow \beta & \pi\left(\delta^{d}\right), \Gamma \rightarrow \beta \\
\pi\left(\delta^{d-1}\right) \vee \pi\left(\delta^{d}\right), \Gamma \rightarrow \beta &
\end{array}
$$

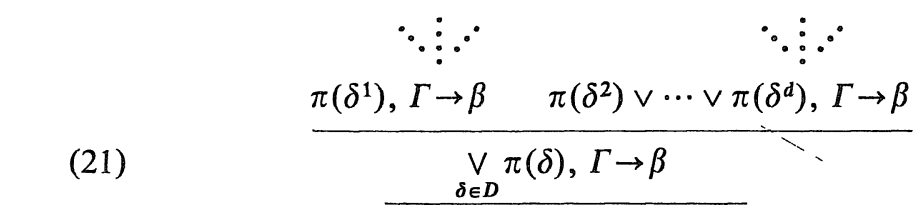

$$
\frac{\pi, \Gamma \rightarrow[O n] \vee_{\delta \in D} \pi(\delta) \quad \overline{[Q n] \vee_{\delta \in D} \pi(\delta), \Gamma \rightarrow[O n] \beta}}{\pi, \Gamma \rightarrow[O n] \beta}
$$

(D2) $\vdash \beta, \pi(\delta), \Gamma \rightarrow$ for some $\delta \in D$ :

In this case, we have the following proof figure:

$$
\begin{gathered}
\because \because \vdots \ominus^{\bullet} \\
\frac{\beta, \pi(\delta), \Gamma \rightarrow}{\beta, \Gamma \rightarrow \neg \pi(\delta)} \\
\frac{(24) \quad \frac{[O n] \beta, \Gamma \rightarrow[O n] \neg \pi(\delta)}{<O n>\pi(\delta),[O n] \beta, \Gamma \rightarrow}}{[O n] \beta, \pi, \Gamma \rightarrow}
\end{gathered}
$$

This completes the proof of Lemma 6.9.

Suggested by this lemma, we construct a KT5-model $M=<E_{0}$; $r, v>$ as follows: 
(i) $(\varepsilon, \delta) \in r\left(S_{i}, n\right)$ iff

(a) $\varepsilon=\delta$

or

(b) $\varepsilon \oplus \delta=e_{i}$ and $n<\|\varepsilon(+i)\|=\|\delta(+i)\|$.

(ii) $(\varepsilon, \delta) \in r(0, n)$ iff

(c) $\varepsilon=\delta$

or

(d) $n<\max \{\|\varepsilon(+i)\| \mid i=1, \ldots, k\}$ and

$n<\max \{\|\delta(+i)\| \mid i=1, \ldots, k\}$.

(iii) $\varepsilon \in v\left(p_{i}\right)$ iff $\varepsilon_{i}=+$.

(iv) $v(\perp)=\emptyset$.

As an example, we illustrate $M$ for $k=3$

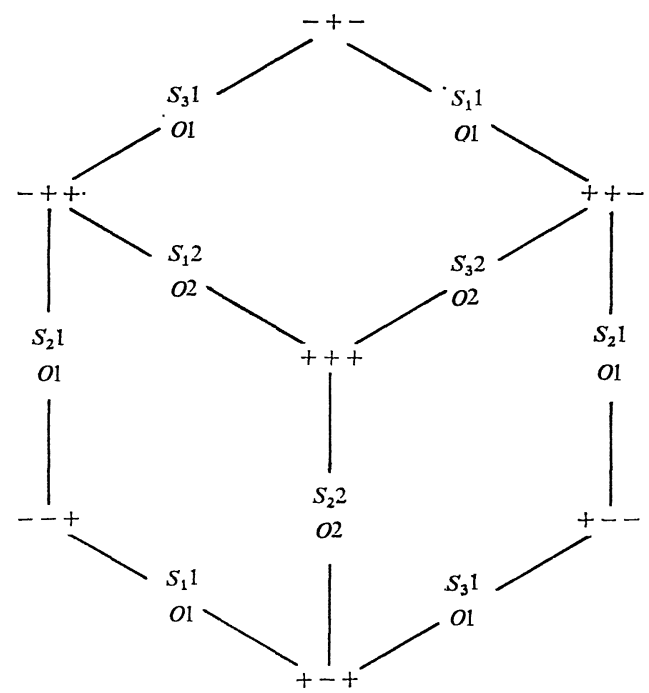

Fig. 6.3. Structure of $M$ for $k=3$

The following lemma shows that $M$ is a model of $\Gamma$.

Lemma 6.10. Let $\varepsilon \in E_{0}$ and $\alpha \in \mathrm{Wff}$. Then we have $\vdash \pi(\varepsilon), \Gamma \rightarrow \alpha$ if and only if $\varepsilon \vDash \alpha($ in $M)$.

Proof. The proof is obtained by faithfully tracing the proof of Lemma 6.9. We prove that (a) $\varepsilon \vDash \alpha$ implies $\vdash \pi(\varepsilon), \Gamma \rightarrow \alpha$ and (b) $\varepsilon=\alpha$ implies $\vdash \alpha, \pi(\varepsilon), \Gamma \rightarrow$, by induction on the construction of $\alpha$. How- 
ever, we only prove the case $\alpha=[O n] \beta$ since other cases may be dealt with similarly by referring to the proof of Lemma 6.9 .

Proof of (a).

Suppose $\varepsilon \models[O n] \beta$. We have two cases.

(A) The case $n \geq \max \{\|\varepsilon(+i)\| \mid i=1, \ldots, k\}$ :

Since $\varepsilon \vDash \beta$, we have

$$
\vdash \pi(\varepsilon), \Gamma \rightarrow \beta
$$

by induction hypothesis. Together with (13) or (17) in Lemma 6.9, we have:

$$
\begin{array}{cc}
\text { (13) or }(17) & \pi(\varepsilon), \Gamma \rightarrow \beta \\
\frac{\pi(\varepsilon), \Gamma \rightarrow[O n] \pi(\varepsilon)}{[O n] \pi(\varepsilon), \Gamma \rightarrow[O n] \beta}
\end{array}
$$

(B) The case $n<\max \{\|\varepsilon(+i)\| \mid i=1, \ldots, k\}$ :

Let $D_{n}=\left\{\delta \in E_{0} \mid n<\max \{\|\delta(+i)\| \mid i=1, \ldots, k\}\right\}$. By the definition of $r$, we have $\varepsilon \stackrel{o_{n}}{\longrightarrow} \delta$ for any $\delta \in D_{n}$. Then we have $\delta \vDash \beta$, since $\varepsilon \vDash[O n] \beta$. Hence, by induction hypothesis, we have

$$
\vdash \pi(\delta), \Gamma \rightarrow \beta
$$

for all $\delta \in D_{n}$. Then we have

$$
\vdash \pi(\varepsilon), \Gamma \rightarrow[O n] \beta
$$

by (25) in Lemma 6.9.

Proof of (b).

Suppose $\varepsilon=[O n] \beta$. We have some $\delta$ such that $\delta=\beta$ and $\varepsilon \stackrel{o_{n}}{\longrightarrow} \delta$.

(C) The case $n \geq \max \{\|\varepsilon(+i)\| \mid i=1, \ldots, k\}$ :

In this case, by the definition of $r$, we have $\delta=\varepsilon$. So, we have

$$
\vdash \beta, \pi(\varepsilon), \Gamma \rightarrow
$$

by induction hypothesis. Hence we have 


$$
\vdash[O n] \beta, \pi(\varepsilon), \Gamma \rightarrow
$$

(D) The case $n<\max \{\|\varepsilon(+i)\| \mid i=1, \ldots, k\}$ :

By the definition of $r$, we have $\delta \in D_{n}$. Then, by (26) in Lemma 6.9 , we have

$$
\vdash[O n] \beta, \pi(\varepsilon), \Gamma \rightarrow
$$

Lemma 6.11. Let $\varepsilon \in E_{0}$ and $\alpha \in \mathrm{Wff}$. Then we have $B_{\pi(\varepsilon)}\left(S_{i} n\right) \vdash \alpha$ if and only if $\varepsilon \vDash\left[S_{i} n\right] \alpha$.

Proof. Only if part: Suppose $B_{\pi(\varepsilon)}\left(S_{i} n\right) \vdash \alpha$. Then we have $B_{\pi(\varepsilon)}\left(S_{i} n\right) \vdash\left[S_{i} n\right] \alpha$. Hence, we have

$$
[O 1]\left(T \supset[O 1]\left(\pi(\varepsilon) \supset\left[S_{i} n\right] \alpha\right)\right) \in \Gamma \text {. }
$$

From this we see that

$$
\vdash \pi(\varepsilon), \Gamma \rightarrow\left[S_{i} n\right] \alpha .
$$

Hence, by the above lemma, we have $\varepsilon \vDash\left[S_{i} n\right] \alpha$.

If part: We have two cases.

(A) $n \geq\|\varepsilon(+i)\|:$ Since $\left[S_{i} n\right]\left[S_{i} n-1\right] \cdots\left[S_{\imath} 1\right] p_{j}^{\varepsilon_{J}} \in B_{\pi(\varepsilon)}\left(S_{i} n\right)$ for any $j \neq i$, and $B_{\pi(\varepsilon)}\left(S_{i} n\right) \vdash p_{i}^{\varepsilon_{i}}$ (Lemma 6.7), we have

$$
\vdash B_{\pi(\varepsilon)}\left(S_{i} n\right) \rightarrow \pi(\varepsilon) .
$$

Since $\varepsilon \vDash\left[S_{i} n\right] \alpha$, we have

$$
\vdash \pi(\varepsilon), \Gamma \rightarrow\left[S_{i} n\right] \alpha
$$

by Lemma 6.10 . Thus we obtain the following proof figure:

$$
\begin{gathered}
\because \vdots 0^{\circ} \quad \begin{array}{l}
\ddots \\
B_{\pi(\varepsilon)}\left(S_{i} n\right) \rightarrow \pi(\varepsilon) \quad \pi(\varepsilon), \Gamma \rightarrow\left[S_{i} n\right] \alpha
\end{array} \\
\frac{B_{\pi(\varepsilon)}\left(S_{i} n\right), \Gamma \rightarrow\left[S_{i} n\right] \alpha}{B_{\pi(\varepsilon)}\left(S_{i} n\right),\left[S_{i} 1\right] \Gamma \rightarrow\left[S_{i} n\right] \alpha} \\
\vdots \\
\frac{B_{\pi(\varepsilon)}\left(S_{i} n\right),\left[S_{i} n\right] \cdots\left[S_{i} 1\right] \Gamma \rightarrow\left[S_{i} n\right] \alpha}{B_{\pi(\varepsilon)}\left(S_{i} n\right) \rightarrow\left[S_{i} n\right] \alpha} \quad \text { (extension) }
\end{gathered}
$$


(B) $n<\|\varepsilon(+i)\|$ : Let $\delta=\varepsilon \oplus c_{i}$. Since $\varepsilon \stackrel{s_{i n}}{\longrightarrow} \delta$, we have $\delta \vDash\left[S_{i} n\right] \alpha$. Hence we have the following proof figure:

$$
\begin{aligned}
& \because \vdots 0^{\circ} \quad \because \because 0^{\circ} \\
& \because \vdots \ominus^{\circ} \quad \pi(\varepsilon), \Gamma \rightarrow\left[S_{i} n\right] \alpha \quad \pi(\delta), \Gamma \rightarrow\left[S_{i} n\right] \alpha \\
& B_{\pi(\varepsilon)}\left(S_{i} n\right) \rightarrow \pi(\varepsilon) \vee \pi(\delta) \quad \pi(\varepsilon) \vee \pi(\delta), \Gamma \rightarrow\left[S_{i} n\right] \alpha \\
& B_{\pi(\varepsilon)}\left(S_{i} n\right), \Gamma \rightarrow\left[S_{i} n\right] \alpha \\
& B_{\pi(\varepsilon)}\left(S_{i} n\right),\left[S_{i} n\right] \cdots\left[S_{i} 1\right] \Gamma \rightarrow\left[S_{i} n\right] \alpha \\
& B_{\pi(\varepsilon)}\left(S_{i} n\right) \rightarrow\left[S_{i} n\right] \alpha
\end{aligned}
$$

Combining the above two lemmas, we have

Corollary 6.12. Let $\varepsilon \in E_{0}$ and $\alpha \in \mathrm{Wff}$. Then we have $B_{\pi(\varepsilon)}\left(S_{i} n\right) \vdash \alpha$ if and only if $\vdash \pi(\varepsilon), \Gamma \rightarrow\left[S_{i} n\right] \alpha$.

Let us recall here that we have been arguing by assuming that $\left.<\left\langle B_{\pi}\left(S_{i} n\right)\right\rangle, \Gamma\right\rangle$ is a solution of $\$$ satisfying (\#) and (\#\#). By inspecting $E q(*)$, we sec that $\Gamma$ is uniquely determined by Lemma 6.11 (provided that $\left\langle\left\langle B_{\pi}\left(S_{i} n\right)\right\rangle, \Gamma\right\rangle$ is in fact a solution of $\$$ under (\#) and $(\# \#))$. So, let $\tilde{\Gamma} \subseteq \mathrm{Wff}$ be defined by:

$$
\begin{gathered}
\tilde{\Gamma}=\left\{[O 1] \| \bigvee_{i=1}^{k} p_{i}\right\} \cup\left\{[01]\left\{S_{i} 1\right\} p_{j} \mid j \neq i, i=1, \ldots, k, j=1, \ldots, k\right\} \\
\cup\left\{[O 1]\left(\pi \supset\left(P\left(\pi, i, n, p_{i}\right) \supset[O n+1]\left[S_{i} n\right] p_{i}\right)\right) \mid \pi \in \Pi_{0},\right. \\
i=1, \ldots, k, n \in T\} \\
\cup\left\{[O 1]\left(\pi \supset\left(\bar{P}\left(\pi, i, n, p_{i}\right) \supset[O n+1] \neg\left[S_{i} n\right] p_{i}\right)\right) \mid \pi \in \Pi_{0},\right. \\
\quad i=1, \ldots, k, n \in T\} \\
\cup\left\{[O 1]\left(P(\pi, i, n, \alpha) \supset[O 1]\left(\pi \supset\left[S_{i} n\right] \alpha\right)\right) \mid \pi \in \Pi_{0},\right. \\
i=1, \ldots, k, n \in T, \alpha \in \mathrm{Wff}\}
\end{gathered}
$$

where $P$ and $\bar{P}$ are defined by

$$
P(\pi(\varepsilon), i, n, \alpha)= \begin{cases}T & \text { if } \varepsilon \vDash\left[S_{i} n\right] \alpha \\ \perp & \text { otherwise }\end{cases}
$$


and

$$
\bar{P}(\pi(\varepsilon), i, n, \alpha)= \begin{cases}\top & \text { if } \varepsilon=\mid\left[S_{i} n\right] \alpha \\ \perp & \text { otherwise. }\end{cases}
$$

Using this $\tilde{\Gamma}$, we define $\widetilde{B}_{\pi}\left(S_{i} n\right)$ inductively by means of equations:

$$
\begin{aligned}
\widetilde{B}_{\pi}\left(S_{i} 1\right)=\left[S_{i} 1\right] \tilde{\Gamma} \cup\left\{\left[S_{i} 1\right] p_{j}^{\xi_{j}} \mid j \neq i, j=1, \ldots, k\right\}, \\
\widetilde{B}_{\pi}\left(S_{i} n+1\right)=\left[S_{i} n+1\right] \widetilde{B}_{\pi}\left(S_{i} n\right) \\
\cup\left\{\left[S_{i} n+1\right]\left[S_{j} n\right] p_{j} \mid \widetilde{B}_{\pi}\left(S_{j} n\right) \vdash p_{j}, j=1, \ldots, k\right\} \\
\cup\left\{\left[S_{i} n+1\right] \neg\left[S_{j} n\right] p_{j} \mid \widetilde{B}_{\pi}\left(S_{j} n\right) \vdash p_{j}, j=1, \ldots, k\right\},
\end{aligned}
$$

where $\pi=\pi(\varepsilon)$.

In order to show that thus defined $<<\widetilde{B}_{\pi}\left(S_{i} n\right)>, \tilde{\Gamma}>$ is the unique solution of $\$$ under $(\#)$ and $(\# \#)$, we prepare several lemmas.

Lemma 6.13. $\tilde{\Gamma}$ satisfies (\#), i.e., for any $\varepsilon \in E_{0},\{\pi(\varepsilon)\} \cup \tilde{\Gamma}$ is consistent.

Proof. It suffices to prove that $\varepsilon \models\{\pi(\varepsilon)\} \cup \tilde{\Gamma}$ (in $M$ ). It is clear that $\varepsilon \vDash \pi(\varepsilon)$. It remains to show that $\varepsilon \models \tilde{\Gamma}$. However, we only prove (a) $\varepsilon \vDash[O 1]\left(\pi \supset\left(P\left(\pi, i, n, p_{i}\right) \supset[O n+1]\left[S_{i} n\right] p_{i}\right)\right)$ and (b) $\varepsilon \vDash[O 1](\pi \supset(P(\pi, i, n$, $\left.\left.p_{i}\right) \supset[O n+1] \neg\left[S_{i} n\right] p_{i}\right)$ ), and leave the verification of remaining parts to the reader.

Proof of (a).

Take any $\delta \in E_{0}$ such that $\varepsilon \stackrel{O 1}{\longrightarrow} \delta$ and suppose that $\delta \models \pi$ and $\delta \vDash P\left(\pi, i, n, p_{i}\right)$. Then we have $\pi=\pi(\delta)$ and $\delta \vDash\left[S_{i} n\right] p_{i}$. Suppose, by way of contradiction, that there is a $\gamma \in E_{0}$ such that $\delta \stackrel{o_{n}+1}{\longrightarrow} \gamma$ and $\gamma=$ $\left[S_{i} n\right] p_{i}$. Then we have $\gamma \neq \delta$ and hence $n+1<\max \{\|\delta(+l)\| \mid l=1, \ldots, k\}$. Hence, $n<\|\delta(+i)\|$. But, since $\delta \models\left[S_{i} n\right] p_{i}$, we have $n \geq\|\delta(+i)\|$, which is a contradiction.

Proof of (b).

Take any $\delta$ such that $\varepsilon \stackrel{o 1}{\longrightarrow} \delta$ and suppose that $\delta \models \pi$ and $\delta \vDash \bar{P}(\pi, i$, $\left.n, p_{i}\right)$. Then we have $\pi=\pi(\delta)$ and $\delta=\left[S_{i} n\right] p_{i}$. Suppose further that there is a $\gamma \in E_{0}$ such that $\delta \stackrel{o_{n+1}}{\longrightarrow} \gamma$ and $\gamma \vDash\left[S_{i} n\right] p_{i}$. Then we have $\gamma \neq \delta$ and hence $n+1<\max \{\|\gamma(+l)\| \mid l=1, \ldots, k\}$. Hence, $n<\|\gamma(+i)\|$. But, 
since $\gamma \vDash\left[S_{i} n\right] p_{i}$, we have $n \geq\|\gamma(+i)\|$. This is a contradiction. Thus, we see $\delta \models[O n+1] \neg\left[S_{\imath} n\right] p_{i}$.

Parallel to Lemma 6.9, we have the following lemma.

Lemma 6.14. Let $\varepsilon \in E_{0}$ and $\pi=\pi(\varepsilon)$. Then, for any $\alpha \in \mathrm{Wff}$, we have either $\vdash \pi, \tilde{\Gamma} \rightarrow \alpha$ or $\vdash \alpha, \pi, \tilde{\Gamma} \rightarrow$.

Proof. By a slight modification, the proof goes exactly parallel to that of Lemma 6.9. For example, in place of (6) in Lemma 6.9, we obtain

$$
\vdash \pi, \tilde{\Gamma} \rightarrow<S_{i} n>\pi\left(\varepsilon^{\prime}\right)
$$

by the following reasoning: Suppose $\pi=\pi(+i)$. Then, since $n<\|\varepsilon(+i)\|$, we have $\varepsilon \models\left[S_{i} n\right] \neg\left[S_{i} n\right] p_{i}$ (by the definition of $M$ ). Then, by the definition of $\tilde{\Gamma}$, we see that

$$
[O 1]\left(\top \supset[O 1]\left(\pi \supset\left[S_{i} n\right] \neg\left[S_{i} n\right] p_{i}\right)\right) \in \tilde{\Gamma} .
$$

Now the proof of ( $\tilde{6})$ goes completely parallel to the proof of (6) in Lemma 6.9.

The following lemma may also be proved parallel to Lemma 6.10 .

Lemma 6.15. Let $\varepsilon \in E_{0}$ and $\alpha \in \mathrm{Wff}$. Then we have $\vdash \pi(\varepsilon), \tilde{\Gamma} \rightarrow \alpha$ if and only if $\varepsilon \vDash \alpha$.

We next prove the analogue of Lemma 6.11 .

Lemma 6.16. Let $\varepsilon \in E_{0}$ and $\alpha \in \mathrm{Wff}$. Then we have $\widetilde{B}_{\pi(\varepsilon)}\left(S_{i} n\right) \vdash \alpha$ if and only if $\varepsilon \vDash\left[S_{i} n\right] \alpha$.

Proof. We prove the following three propositions by induction on $n$.

$\left(A_{n}\right) \quad \widetilde{B}_{\pi(\varepsilon)}\left(S_{i} n\right) \vdash \alpha$ implies $\varepsilon \models\left[S_{i} n\right] \alpha$.

$\left(B_{n}\right) \quad n \geq\|\varepsilon(+i)\|$ implies $\widetilde{B}_{\pi(+i)}\left(S_{i} n\right) \vdash p_{i}$ and $\widetilde{B}_{\pi(-i)}\left(S_{i} n\right) \vdash \bar{p}_{i}$ (if $\left.\pi(-i) \in \Pi_{0}\right)$.

$\left(C_{n}\right) \quad \varepsilon \models\left[S_{i} n\right] \alpha$ implies $\widetilde{B}_{\pi(\varepsilon)}\left(S_{i} n\right) \vdash \chi$. 
We first remark that to prove $\left(A_{n}\right)$ it is sufficient to prove:

$\left(A_{n}^{\prime}\right) \quad \varepsilon \vDash \widetilde{B}_{\pi(\varepsilon)}\left(S_{i} n\right)$.

For, suppose $\varepsilon \vDash \widetilde{B}_{\pi(\varepsilon)}\left(S_{i} n\right)$ and $\widetilde{B}_{\pi(\varepsilon)}\left(S_{i} n\right) \vdash \alpha$. Then we have $\vdash \widetilde{B}_{\pi(\varepsilon)}\left(S_{i} n\right)$ $\rightarrow \alpha$, and hence $\vdash \widetilde{B}_{\pi(\varepsilon)}\left(S_{i} n\right) \rightarrow\left[S_{i} n\right] \alpha$ (by $\left(\rightarrow n,\left[S_{i} n\right]\right)$ ). Since $\varepsilon \vDash \widetilde{B}_{\pi(\varepsilon)}\left(S_{i} n\right)$, we have $\varepsilon \vDash\left[S_{i} n\right] \alpha$ by the Soundness Theorem.

$n=1$ :

Proof of $\left(A_{1}^{\prime}\right) . \quad \varepsilon \models \widetilde{B}_{\pi(\varepsilon)}\left(S_{i} 1\right)$ is easily verified since $\varepsilon \models \tilde{\Gamma}$ and $\vdash \beta \rightarrow\left[S_{i} 1\right] \beta$ for any $\beta \in \tilde{\Gamma}$.

Proof of $\left(B_{1}\right)$. This is proved just as in Lemma 6.7 .

Proof of $\left(C_{1}\right)$. This is proved similarly as in Lemma 6.11 by means of $\left(B_{1}\right)$ in place of Lemma 6.7 and Lemma 6.15 in place of Lemma 6.10.

$n>1$ :

Proof of $\left(A_{n}^{\prime}\right)$. That $\varepsilon \models\left[S_{i} n\right] \widetilde{B}_{\pi(\varepsilon)}\left(S_{i} n-1\right)$ easily follows from $\left(A_{n-1}^{\prime}\right)$. Next, suppose that $\widetilde{B}_{\pi(\varepsilon)}\left(S_{j} n-1\right) \vdash p_{j}$. By $\left(A_{n-1}\right)$ we have

$$
\varepsilon \vDash\left[S_{j} n-1\right] p_{j}
$$

Hence, by the definition of $M$, we have $\varepsilon \vDash p_{j}$ and

$$
n-1 \geq\|\varepsilon(+j)\|=\|\varepsilon\| \text {. }
$$

Suppose $\varepsilon=\left[S_{i} n\right]\left[S_{j} n-1\right] p_{j}$. Then, for some $\delta$ such that $\varepsilon \stackrel{S_{i n}}{\longrightarrow} \delta$, we have

$$
\delta=\left[S_{j} n-1\right] p_{j}
$$

From (1) and (3), we see that $\varepsilon \neq \delta$, and hence $n<\|\varepsilon(+i)\|$. This means

$$
n-1<\|\varepsilon\|
$$

which contradicts (2). Thus we have shown that

$$
\varepsilon \vDash\left[S_{i} n\right]\left[S_{j} n-1\right] p_{j}
$$

Suppose now $\widetilde{B}_{\pi(\varepsilon)}\left(S_{j} n-1\right) \times p_{j}$. Then we have

$$
\varepsilon=\left[S_{j} n-1\right] p_{j}
$$

by $\left(C_{n-1}\right)$. By (4) and by the definition of $M$, we have 


$$
n-1<\|\varepsilon(+j)\|
$$

By way of contradiction, let us suppose $\varepsilon=1\left[S_{i} n\right] \neg\left[S_{j} n-1\right] p_{j}$. Then, for some $\delta$ such that $\varepsilon \stackrel{s_{i n}}{\longrightarrow} \delta$, we have

$$
\delta \models\left[S_{j} n-1\right] p_{j} .
$$

By (4) and (6), we have $\delta=\varepsilon \oplus e_{i}$. By (6) we see that

$$
n-1 \geq\|\delta(+j)\|
$$

By (5) and (7), we have $\|\varepsilon(+j)\|>\|\delta(+j)\|$. Hence we see that $i \neq j$ and $\varepsilon(+i)=\varepsilon$. Now, since $\varepsilon \neq \delta$ and $\varepsilon \stackrel{s_{i n}}{\longrightarrow} \delta$, we have

$$
n<\|\varepsilon(+i)\|=\|\varepsilon\| \text {. }
$$

On the other hand, from (6) we have $n-1 \geq\|\delta(+j)\|$. Hence

$$
n \geq\|\delta(+j)(+i)\|=\|\varepsilon(+j)\|=\|\varepsilon\|
$$

which contradicts (8). Therefore we see that $\varepsilon \models\left[S_{i} n\right] \neg\left[S_{j} n-1\right] p_{j}$ if $\widetilde{B}_{\pi(\varepsilon)}\left(S_{j} n-1\right) \uparrow p_{j}$.

Proof of $\left(B_{n}\right)$. First we show that $\widetilde{B}_{\pi(+i)}\left(S_{i} n\right) \vdash p_{i}$ from the assumption that $n=\|\varepsilon(+i)\|$. Since $n>1$, we can take a $j \neq i$ such that $\varepsilon_{j}=+$. Then $\|\varepsilon(+i)(+j)\|=n>n-1$. Hence we have $\varepsilon(+i)=\left[S_{j} n-1\right] p_{j}$. So, by $\left(A_{n-1}\right)$, we have $\widetilde{B}_{\pi(+i)}\left(S_{j} n-1\right) \ngtr p_{j}$. Hence,

$$
\left[S_{i} n\right] \neg\left[S_{j} n-1\right] p_{j} \in \widetilde{B}_{\pi(+i)}\left(S_{i} n\right) .
$$

Since $\|\varepsilon(-i)(+j)\|=n-1$, we have $\varepsilon(-i) \vDash\left[S_{j} n-1\right] p_{j}$. Hence, by $\left(C_{n-1}\right)$, we have $\widetilde{B}_{\pi(-i)}\left(S_{j} n-1\right) \vdash p_{j}$. Hence, we have $P\left(\pi(-i), j, n-1, p_{j}\right)=\mathrm{T}$, so that

$$
[O 1]\left(\pi(-i) \supset\left(T \supset[O n]\left[S_{j} n-1\right] p_{j}\right)\right) \in \tilde{\Gamma} .
$$

From (9) and (10), we have $\widetilde{B}_{\pi(+i)}\left(S_{i} n\right) \vdash \neg \pi(-i)$. Since $\widetilde{B}_{\pi(+i)}\left(S_{i} n\right)$ $\vdash \pi(+i) \vee \pi(-i)$, we see, $\widetilde{B}_{\pi(+i)}\left(S_{i} n\right) \vdash \pi(+i)$. Hence $\widetilde{B}_{\pi(+i)}\left(S_{i} n\right) \vdash p_{i}$.

The proof of $\widetilde{B}_{\pi(-i)}\left(S_{i} n\right) \vdash \overline{p_{i}}$ from the assumption that $n=\|\varepsilon(+i)\|$ is obtained similarly by modifying the corresponding proof of Lemma 6.7.

The case $n>\|\varepsilon(+i)\|$ is now easy.

Proof of $\left(C_{n}\right)$. Similar to the proof of $\left(C_{1}\right)$. 


\section{Corollary 6.17.}

$$
P(\pi, i, n, \alpha)=\top \quad \text { if and only if } \widetilde{B}_{\pi}\left(S_{i} n\right) \vdash \alpha .
$$

By Lemma 6.5, we also have the following corollary.

Corollary 6.18. $\quad \widetilde{B}_{\pi}\left(S_{i} n\right)$ is a knowledge base for $S_{i} n$.

By Corollary 6.17 , we see that $\left.\left\langle<\widetilde{B}_{\pi}\left(S_{i} n\right)\right\rangle, \tilde{\Gamma}\right\rangle$ is indeed a solution of $\$$. Furthermore, by Lemma 6.13 and Corollary 6.18, we see that $<<\widetilde{B}_{\pi}\left(S_{i} n\right)>, \tilde{\Gamma}>$ satisfies $(\#)$ and (\#\#). Since we already know that $\$$ has at most one solution under $(\#)$ and $(\#)$, we have thus established the following theorem.

Theorem 6.19. Under the conditions (\#) and (\#\#), \$ has the unique solution $<<\widetilde{B}_{\pi}\left(S_{i} n\right)>, \tilde{\Gamma}>$.

Thus we have seen that $\tilde{\Gamma}$ may be regarded as the formal counterpart of the King's order in our formal system. The puzzle is then reduced to the problem of showing that:

$\left(P_{1}\right)$ If $\|\varepsilon\|=n$ and $\varepsilon_{l}=+$, then $\widetilde{B}_{\pi(\varepsilon)}\left(S_{i} n\right) \vdash p_{i}$ and $\widetilde{B}_{\pi(\varepsilon)}\left(S_{i} n-1\right) \uparrow p_{i}$.

We note that we can moreover prove the following:

$\left(P_{2}\right) \quad$ If $\|\varepsilon\|=n$ and $\varepsilon_{i}=-$, then $\widetilde{B}_{\pi(\varepsilon)}\left(S_{i} n+1\right) \vdash \overline{p_{i}}$ and $\widetilde{B}_{\pi(\varepsilon)}\left(S_{i} n\right) \vdash \overline{p_{i}}$.

Though Lemma 6.16 gives us a solution to the problems $\left(P_{1}\right)$ and $\left(P_{2}\right)$, we show below a sample proof for the case $k=3$ and $\varepsilon=++-$ :

We put $\pi=\pi(\varepsilon)=p_{1} \wedge p_{2} \wedge \overline{p_{3}}$. Noting that $\left[S_{1} 2\right] \neg\left[S_{2} 1\right] p_{2} \in \widetilde{B}_{\pi}\left(S_{1} 2\right)$ since $\widetilde{B}_{\pi}\left(S_{2} 1\right) \uparrow p_{2}$, and $[O 1]\left(\pi(-+-) \supset\left(\top \supset[O 2]\left[S_{2} 1\right] p_{2}\right)\right) \in \widetilde{\Gamma}$ since $\widetilde{B}_{\pi(-+-)}\left(S_{2} 1\right) \vdash p_{2}$, we can construct a proof of

$$
\widetilde{B}_{\pi}\left(S_{1}\right) \rightarrow p_{1}
$$

as follows. (See Fig. 6.4.)

The model $M=\left\langle E_{0} ; r, v\right\rangle$ has played a crucial role for the solution of $\$$. We wish to point out that $M$ may be considered as essentially the unique and hence the inherent model of $\tilde{\Gamma}$. Let us consider any KT5-model $N=<W_{N} ; r_{N}, v_{N}>$ such that $w_{0} \vDash \tilde{\Gamma}$ (in $N$ ) for some $w_{0} \in W_{N}$. 


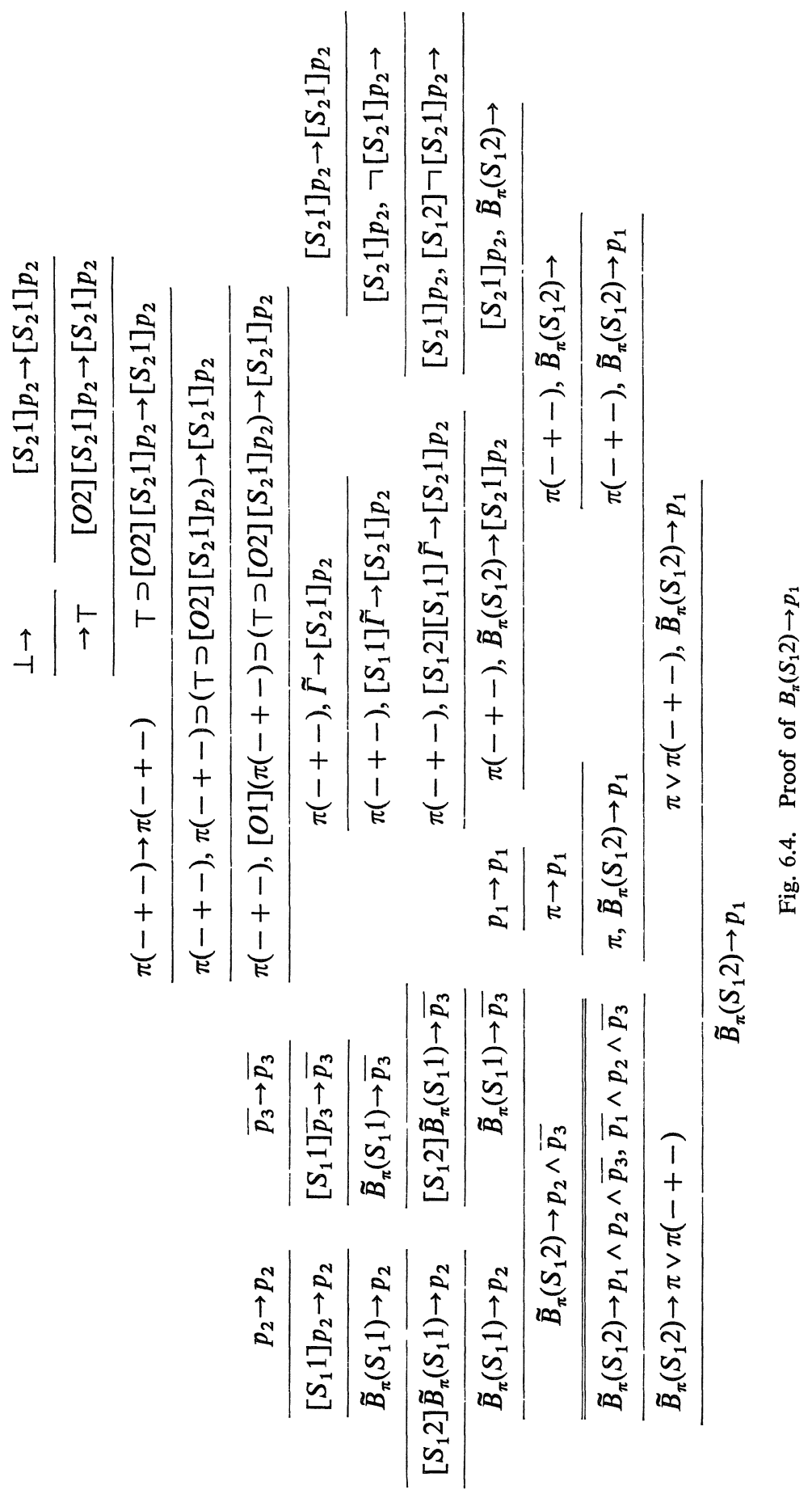


Let $W_{0}=\left\{w \in W_{N} \mid\left(w_{0}, w\right) \in r_{N}(0,1)\right\}$. Then by restricting $r_{N}$ and $v_{N}$ to $W_{0}$, we obtain a model $N_{0}=\left\langle W_{0} ; r_{0}, v_{0}\right\rangle$ and still have $w_{0} \vDash \tilde{\Gamma}$ (in $N_{0}$ ). Let $\tilde{N}_{0}=\overline{N_{0}} / \chi_{N_{0}}$ (where we take relational closure and characteristic function in the category $\left.\mathscr{K}_{5}(\mathrm{Wff})\right)$. Then by Theorem 4.9 , we have that $\tilde{N}_{0}$ is reduced and $\tilde{w}_{0} \vDash \tilde{\Gamma}$ (in $\tilde{N}_{0}$ ). We also have $\tilde{r}_{0}(O, 1)=\widetilde{W}_{0} \times \widetilde{W}_{0}$. Hence we have $w \models \tilde{\Gamma}$ (in $\tilde{N}_{0}$ ) for all $w \in \tilde{W}_{0}$. We will prove that $\tilde{N}_{0}$ is strongly isomorphic to $M$.

First, we define a function

$$
h: \tilde{W}_{0} \longrightarrow E_{0}
$$

by letting $h(w)$ be the unique $\varepsilon \in E_{0}$ such that $w \models \pi(\varepsilon)$ (in $\tilde{N}_{0}$ ). Since $w \models \tilde{\Gamma}$ and [O1] $\vee p_{i} \in \tilde{\Gamma}$, we see that $h$ is well-defined. Let $w \in \tilde{W}_{0}$ and $\varepsilon=h(w)$. Take any formula $\alpha$. Suppose $\varepsilon \models \alpha$ (in $M$ ). Then we have $\vdash \pi(\varepsilon), \tilde{\Gamma} \rightarrow \alpha$ by Lemma 6.15. From this, since $w \models \tilde{\Gamma}$ and $w \models \pi(\varepsilon)$, we have $w \vDash \alpha$. Thus, we see that $h$ is a homomorphism (in $\mathscr{K}_{5}$ (Wff)).

Let $\varepsilon$ be any element in $E_{0}$. Take any $w \in \widetilde{W}_{0}$. Since $\vdash \tilde{\Gamma} \rightarrow$ $<O 1>\pi(\varepsilon)$, we have $w \models<O 1>\pi(\varepsilon)$. Then there is a $w^{\prime} \in \tilde{W}_{0}$ such that $w^{\prime} \vDash \pi(\varepsilon)$. Hence we have $h\left(w^{\prime}\right)=\varepsilon$. Thus we see that $h$ is onto.

Since $\tilde{N}_{0}$ is reduced, $\chi_{\bar{N}_{0}}=\chi_{M^{\circ}} h$ is an injection by Lemmas 4.2 and 4.7. Hence $h$ is also an injection.

Take any $S \in S p$ and $n \in T$. Let $w, w^{\prime} \in \tilde{W}_{0}$. Suppose $w \stackrel{S_{n}}{\longrightarrow} w^{\prime}$. Then $w \vDash<S n>\pi\left(h\left(w^{\prime}\right)\right)$ (in $\left.N_{0}\right)$. Hence $h(w) \vDash<S n>\pi\left(h\left(w^{\prime}\right)\right.$ ) (in $M$ ). This means $h(w) \stackrel{S_{n}}{\longrightarrow} h\left(w^{\prime}\right)$. Next, suppose $h(w) \stackrel{S_{n}}{\longrightarrow} h\left(w^{\prime}\right)$. Then $h(w)$ $\vDash<S n>\pi\left(h\left(w^{\prime}\right)\right)$ (in $\left.M\right)$. Since $h^{-1}$ is a homomorphism, we have $w$ $\vDash<S n>\pi\left(h\left(w^{\prime}\right)\right)$ (in $\left.N_{0}\right)$. Hence there is some $w^{\prime \prime}$ such that $w \stackrel{S n}{\longrightarrow} w^{\prime \prime}$ and $w^{\prime \prime} \vDash \pi\left(h\left(w^{\prime}\right)\right)$. So, we have $h\left(w^{\prime \prime}\right)=h\left(w^{\prime}\right)$. Since $h$ is injective, we have $w^{\prime \prime}=w^{\prime}$, so that $w \stackrel{S_{n}}{\longrightarrow} w^{\prime}$.

Thus we have proved that $\tilde{N}_{0}$ is strongly isomorphic to $M$.

Remark. We can analyze the wise men puzzle furthermore by a method similar to the one we used in this $\S$. We wish to discuss it in a paper to be published jointly with McCarthy et al.

\section{Acknowledgments}

I would like to express my sincerest thanks to Professor John 
McCarthy of Stanford University who has guided me to his ingenious theory of modal axiomatization of knowledge.

I would like to express my hearty thanks to Professor Satoru Takasu for his advice and encouragement.

I would like to express my thanks to Professors Kazuo Matsumoto, Tsutomu Hosoi, Shigeru Igarashi and Hiroakira Ono and to Messrs. Osamu Sonobe, Takeshi Hayashi and Satoru Nagai for their helpful suggestions and kind discussions with me.

\section{References}

[1] Bass, H., Finite monadic algebras, Proc. Amer. Math. Soc., 5 (1958), 258268.

[2] Cresswell, M. J., Frames and models in classical modal logic, in: Algebra and logic, Lecture Notes in Math., 450 (1975), 63-86, Springer, Berlin-HeidelbergNew York.

[3] Fitting, M. C., Intuitionistic logic model theory and forcing, North-Holland, Amsterdam, 1969.

[4] Gentzen, G., Untersuchungen über das logische Schliessen I, II, Math. Z., 39 (1935), 176-210, 405-431.

[5] — Investigations into logical deduction (English translation of [4]), in: Szabo, M. E., ed., The collected papers of Gerhard Gentzen, 68-131, NorthHolland, Amsterdam, 1969.

[6] Gödel, K., Die Vollständigkeit der Axiome des logischen Funktionenkalküls, Monatsh. Math. Phys., 37 (1930), 349-360.

[7] Grätzer, G., Universal algebra, Van Nostrand, Princeton, 1968.

[8] Hayashi, T., Notes on K and KI, private communication, 1975.

[9] — Disjunctive property in McCarthy's propositional knowledge system, private communication, 1976.

[10] Henkin, L., The completeness of the first-order functional calculus, J. Symbolic Logic, 14 (1949), 159-166.

[11] Hintikka, J., Knowledge and Belief, an introduction to the logic of the two notions, Cornell University Press, Ithaca and London, 1962.

[12] Itoh, M., On the relation between the modal sentential logic and monadic predicate calculus I, II, III (in Japanese), J. Japan Assoc. for Philosophy of Sciences, 3, 4, 6 (1955-56), 40-43, 14-19, 18-25.

[13] Kreisel, G., A survey of proof theory, J. Symbolic Logic, 33 (1968), 321388.

[14] —, A survey of proof theory II, in: Festad, J. E., ed., Proceedings of the Second Scandinavian Logic Symposium, North-Holland, Amsterdam, 1971.

[15] Kripke, S., Semantical analysis of modal logic I - normal modal propositional calculi, Z. Math. Logik Grundlagen Math., 9 (1963), 67-96.

[16] —- Semantical analysis of intuitionistic logic I, in: Formal systems and recursive functions, North-Holland, Amsterdam, 1965. 
[17] Lemmon, E. J., Algebraic semantics for modal logics I, II, J. Symbolic Logic, 31 (1966), 46-65, 192-218.

[18] Lemmon, E. J. and Scott, D. S., Intensional logic, preliminary draft of initial chapters by E. J. Lemmon, mimeographed, Stanford University, 1966.

[19] Lyndon, R. C., Notes on Logic, Van Nostrand, Princeton, 1966.

[20] Maehara, S., A general theory of completeness proofs, Ann. of the Assoc. for Philosophy of Science, 3 (1970), 242-256.

[21] McCarthy, J., private communication, 1975.

[22] — - An axiomatization of knowledge and the example of the wise man puzzle, private communication, 1976.

[23] Mitchell, B., Theory of categories, Academic Press, New York and London, 1965.

[24] Ohnishi, M. and Matsumoto, K., Gentzen method in modal calculi, Osaka Math. J., 9 (1957), 113-130 and 11 (1959), 115-120.

[25] Prawitz, D., Natural deduction, a proof-theoretical study, Almqvist \& Wiksell, Stockholm, 1965.

[26] —, Ideas and results in proof theory, in: Fenstad, J. E., ed., Proceedings of the Second Scandinavian Logic Symposium, North-Holland, Amsterdam, 1971.

[27] —- Comments on Gentzen-type procedures and the classical notion of truth, in: Proof Theory Symposion, Kiel 1974, Lecture Notes in Math., 500 (1975), 290-319, Springer, Berlin-Heidelberg-New York.

[28] Rasiowa, H., An algebraic approach to non-classical logics, North-Holland, Amsterdam, 1974.

[29] Rasiowa, H. and Sikorski, R., The mathematics of metamathematics, Monografie Mathemtyczne 41, Warszawa, 1963.

[30] Sato, M., Kripke-type models for McCarthy's propositional knowledge system, unpublished memo, 1975.

[31] Schütte, K., Vollständige Systeme modaler und intuitionistischer Logik, Ergebnisse der Mathematik und ihrer Grenzgebiete, Band 42, Springer, BerlinHeidelberg-New York, 1968.

[32] Scott, D. S., Continuous lattices, in: Toposes, algebraic geometry and logic, Lecture Notes in Math., 274 (1972), 97-136, Springer, Berlin-Heidelberg-New York.

[33] — Data types as lattices, in: Logic conference, Kiel 1974, Lecture Notes in Math., 499 (1975), 579-651, Springer, Berlin-Heidelberg-New York.

[34] Segerberg, K., An essay in classical modal logic, Filosofiska Studier, Uppsala University, 1971.

[35] Smullyan, R. M., First-order logic, Ergebnisse der Mathmatik und ihrer Grenzgebiete, Band 43, Springer, Berlin-Heidelberg-New York, 1968.

[36] Sonobe, O., A note on the modal logic S5, private communication, 1975.

[37] Takahashi, M., A system of simple type theory of Gentzen style with inference on extensionality and the cut-elimination in it, Comm. Math. Univ. Sancti Pauli, 18 (1970), 129-147.

[38] Takeuti, G., Proof theory, North-Holland, Amsterdam, 1975.

[39] Zucker, J., The correspondence between cut-elimination and normalization, Ann. Math. Logic, 7 (1974), 1-155. 